University of Tennessee Health Science Center UTHSC Digital Commons

$12-2008$

\title{
Kinematic Demands of Nucleus Arthroplasty Technology
}

Elizabeth J. Sander

University of Tennessee Health Science Center

Follow this and additional works at: https://dc.uthsc.edu/dissertations

Part of the Surgical Procedures, Operative Commons, and the Therapeutics Commons

\section{Recommended Citation}

Sander, Elizabeth J. , "Kinematic Demands of Nucleus Arthroplasty Technology" (2008). Theses and Dissertations (ETD). Paper 355. http://dx.doi.org/10.21007/etd.cghs.2008.0274.

This Thesis is brought to you for free and open access by the College of Graduate Health Sciences at UTHSC Digital Commons. It has been accepted for inclusion in Theses and Dissertations (ETD) by an authorized administrator of UTHSC Digital Commons. For more information, please contact jwelch30@uthsc.edu. 


\title{
Kinematic Demands of Nucleus Arthroplasty Technology
}

\begin{abstract}
Recent advancements in biomaterial technologies have fostered growth in alternative surgical procedures to fusion surgery for treatment of early stages of degenerative disc disease. One application of immediate interest is that of nucleus arthroplasty (NA). Novel materials are being developed to better match the nonlinear biomechanical properties of the native tissue. The effects of changing the motion segment unit (MSU) properties via surgery (nucleotomy) or placement of a nucleus arthroplasty material, changes the effort or work required to move the altered spine condition through a prescribed kinematic path relative to the intact spine condition. The closer the loading mechanics of the altered spine are to the intact spine condition, the better the likelihood the device will restore the native properties. The objective of this research was to use a new testing protocol to evaluate different designs used in nucleus replacement devices and compare their restorative characteristics to the native tissue.
\end{abstract}

Seven human lumbar MSUs were tested in the harvested, nucleotomy, compliant implanted, and noncompliant implanted spine conditions. The spinal segments were mounted in a spine robot and tested in flexion and extension about six fixed points of rotation located along the centerline of the disc and $5 \mathrm{~mm}$ below the endplate. The spinal MSUs were rotated about the designated fixed points of rotation until a target bending moment of $8 \mathrm{Nm}$ of flexion or extension was reached, or the compressive or shear forces exceeded $500 \mathrm{~N}$. Measurements for all test conditions included the MSU axial force normal to the plane of the disc, shear force along the plane of the disc, sagittal rotation, and sagittal bending moment.

During flexion testing, greater MSU rotation occurred for the nucleotomy condition compared to the harvested and implanted spine conditions for all points of rotation. There were no differences between the harvested and compliant implanted spine condition in the MSU rotations, compressive load, or shear load for all points of rotation. The non-compliant implanted spine condition caused greater compressive and shear forces at the posterior points of rotation. Compared to the other three spine conditions, the nucleotomy spine condition had significantly greater rotation in flexion. In extension testing, greater shear and compressive forces acted on the MSU for the nucleotomy spine condition compared to harvested and implanted spine conditions at central and posterior points.

Denucleating the MSU led to a more destabilized spine condition with greater MSU rotation in flexion and greater disk compression in extension. After implantation of a compliant implant, variation was reduced and the response profile moved towards the harvested state for all test points. Implantation of a noncompliant implant caused an increase in the shear and compressive forces acting across the joint. Since spinal discs and compliant nucleus replacement technologies do not have a prescribed axis of rotation, evaluating the kinematic response at multiple locations of rotation may more effectively characterize the restorative effect of these technologies compared to more traditional in vitro test methods. Overall, this method offers new insight into thoroughly understanding the kinematics response of all types of nucleus arthroplasty technologies.

\section{Document Type}

Thesis

\section{Degree Name}

Master of Science (MS)

\section{Program}

Biomedical Engineering and Imaging 


\section{Research Advisor}

Denis DiAngelo Ph.D.

\section{Keywords}

disc degeneration, lumbar spine, nucleus arthroplasty, spine biomechanics

\section{Subject Categories}

Analytical, Diagnostic and Therapeutic Techniques and Equipment | Medicine and Health Sciences | Surgical Procedures, Operative I Therapeutics 


\title{
KINEMATIC DEMANDS OF NUCLEUS ARTHROPLASTY TECHNOLOGY
}

\author{
A Thesis \\ Presented for \\ The Graduate Studies Council \\ The University of Tennessee \\ Health Science Center
}

\author{
In Partial Fulfillment \\ Of the Requirements for the Degree \\ Master of Science \\ In the Joint Graduate Program in Biomedical Engineering and Imaging \\ From The University of Tennessee \\ and \\ The University of Memphis
}

By

Elizabeth J. Sander

December 2008 
Copyright $@ 2008$ by Elizabeth J. Sander All rights reserved. 


\section{ACKNOWLEDGEMENTS}

I would like to thank all the people that were a part of my graduate school experience. First, I would like to thank my advisor, Denis DiAngelo, for all of his input, help, experience, and for allowing me to discover my full abilities as both a researcher and engineer. I would also like to thank my committee members, Dr. Brian Kelly, Dr. Richard Kasser, and Dr. Gladius Lewis, for their feedback and recommendations regarding the research. My deepest appreciation goes to my parents, Dr. Paul and Sylvia Sander, for their love and support of me throughout my educational career and for pushing me to achieve my fullest potential. To all my family and friends, thank you for being there for me and keeping me grounded. I would also like to thank my research

partner, Nephi Zufelt, for his invaluable assistance. Finally, thank you to the students of the Joint and Implant Biomechanics Lab. 


\begin{abstract}
Recent advancements in biomaterial technologies have fostered growth in alternative surgical procedures to fusion surgery for treatment of early stages of degenerative disc disease. One application of immediate interest is that of nucleus arthroplasty (NA). Novel materials are being developed to better match the nonlinear biomechanical properties of the native tissue. The effects of changing the motion segment unit (MSU) properties via surgery (nucleotomy) or placement of a nucleus arthroplasty material, changes the effort or work required to move the altered spine condition through a prescribed kinematic path relative to the intact spine condition. The closer the loading mechanics of the altered spine are to the intact spine condition, the better the likelihood the device will restore the native properties. The objective of this research was to use a new testing protocol to evaluate different designs used in nucleus replacement devices and compare their restorative characteristics to the native tissue.
\end{abstract}

Seven human lumbar MSUs were tested in the harvested, nucleotomy, compliant implanted, and non-compliant implanted spine conditions. The spinal segments were mounted in a spine robot and tested in flexion and extension about six fixed points of rotation located along the centerline of the disc and $5 \mathrm{~mm}$ below the endplate. The spinal MSUs were rotated about the designated fixed points of rotation until a target bending moment of $8 \mathrm{Nm}$ of flexion or extension was reached, or the compressive or shear forces exceeded $500 \mathrm{~N}$. Measurements for all test conditions included the MSU axial force normal to the plane of the disc, shear force along the plane of the disc, sagittal rotation, and sagittal bending moment.

During flexion testing, greater MSU rotation occurred for the nucleotomy condition compared to the harvested and implanted spine conditions for all points of rotation. There were no differences between the harvested and compliant implanted spine condition in the MSU rotations, compressive load, or shear load for all points of rotation. The non-compliant implanted spine condition caused greater compressive and shear forces at the posterior points of rotation. Compared to the other three spine conditions, the nucleotomy spine condition had significantly greater rotation in flexion. In extension testing, greater shear and compressive forces acted on the MSU for the nucleotomy spine condition compared to harvested and implanted spine conditions at central and posterior points.

Denucleating the MSU led to a more destabilized spine condition with greater MSU rotation in flexion and greater disk compression in extension. After implantation of a compliant implant, variation was reduced and the response profile moved towards the harvested state for all test points. Implantation of a non-compliant implant caused an increase in the shear and compressive forces acting across the joint. Since spinal discs and compliant nucleus replacement technologies do not have a prescribed axis of rotation, evaluating the kinematic response at multiple locations of rotation may more effectively characterize the restorative effect of these technologies compared to more traditional in vitro test methods. Overall, this method offers new insight into thoroughly understanding the kinematics response of all types of nucleus arthroplasty technologies. 


\section{TABLE OF CONTENTS}

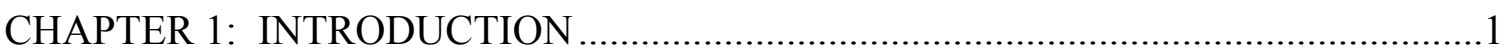

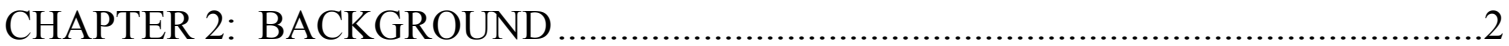

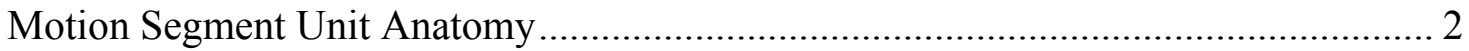

General Spine Anatomy ............................................................................. 2

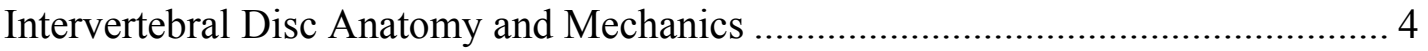

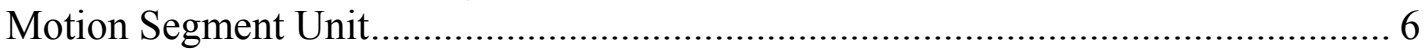

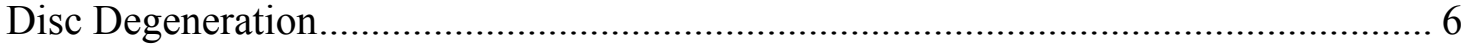

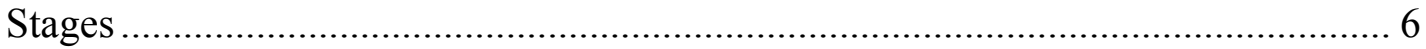

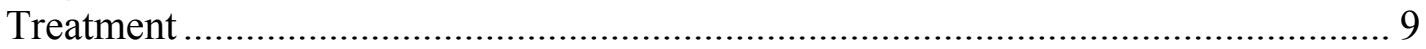

\section{CHAPTER 3: BIOMECHANICAL PERFORMANCE OF A COMPLIANT}

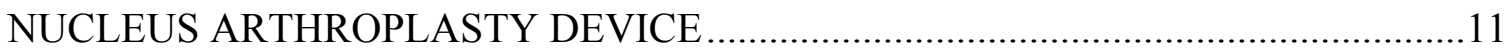

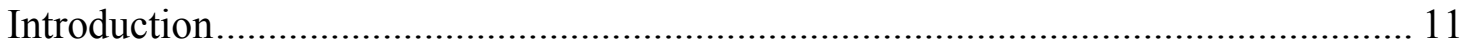

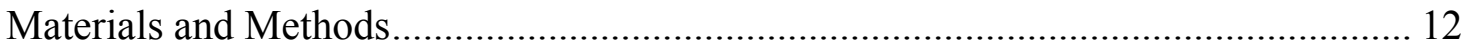

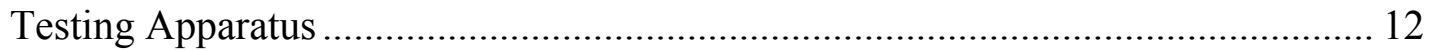

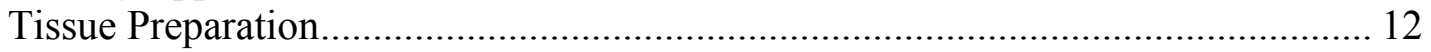

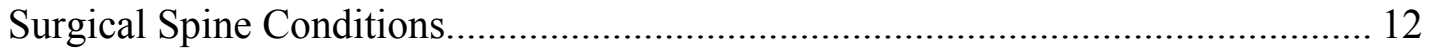

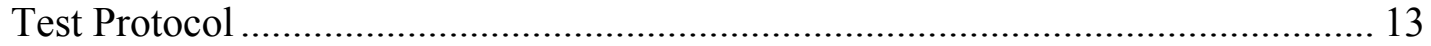

Simulation of Different Centers of Rotation...................................................... 13

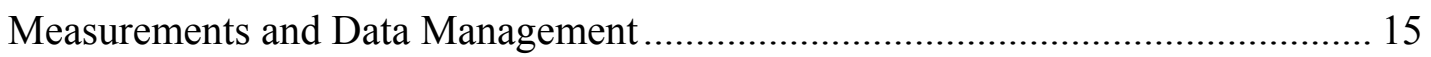

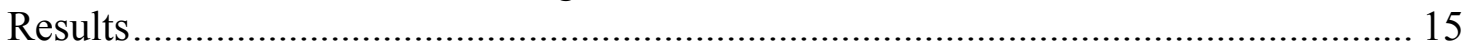

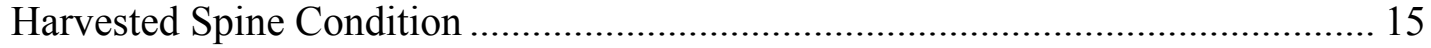

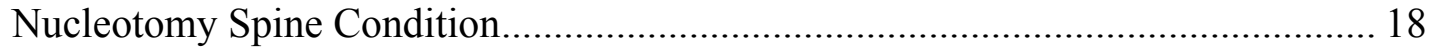

Implanted Spine Condition .............................................................................. 18

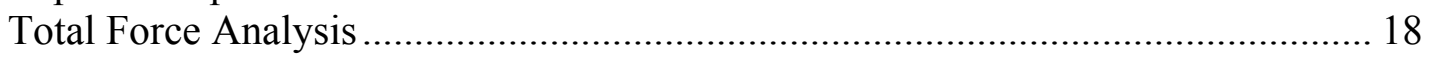

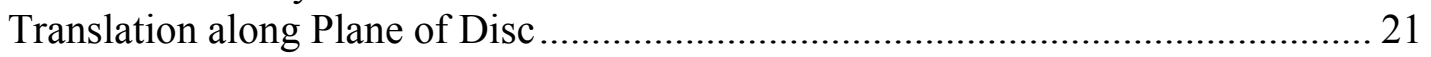

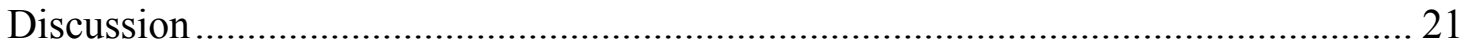

\section{CHAPTER 4: COMPARISON OF COMPLIANT AND NON-COMPLIANT}

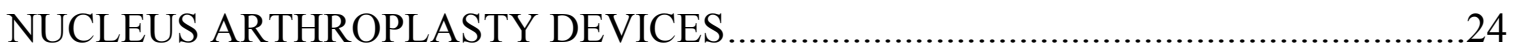

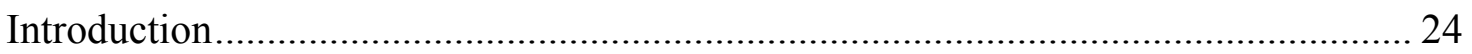

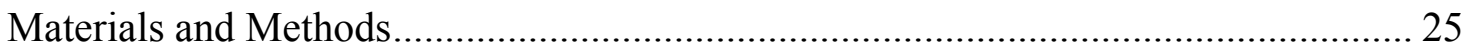

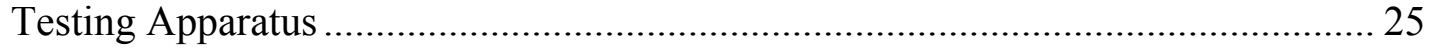

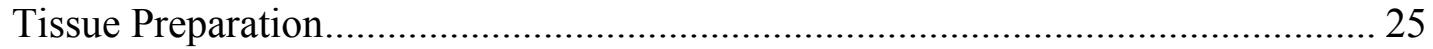

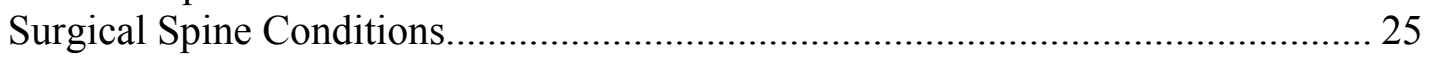

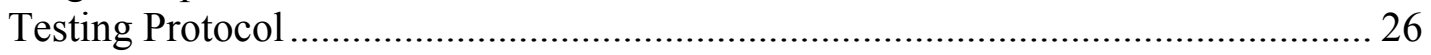

Simulation of Different Centers of Rotation........................................................ 26

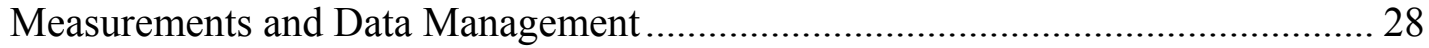

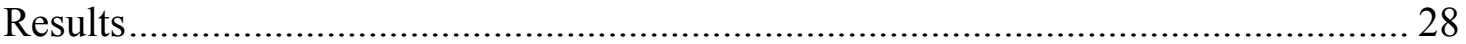

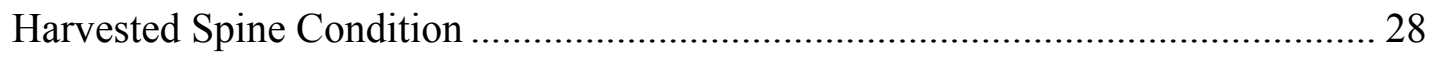

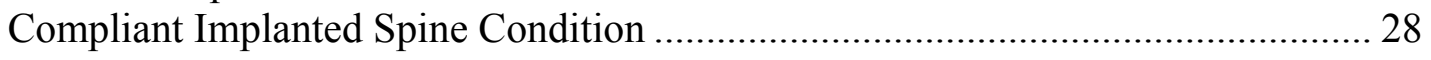

Non-Compliant Implanted Spine Condition ....................................................... 31

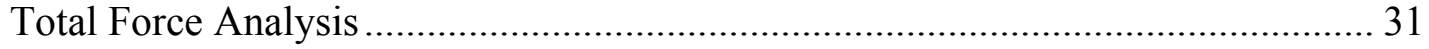




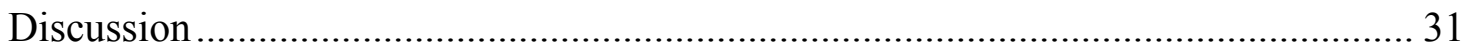

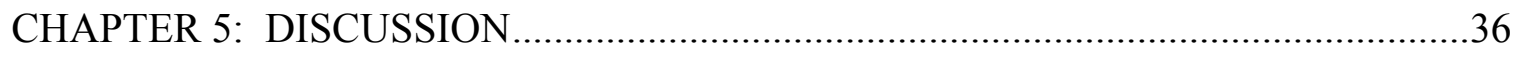

CHAPTER 6: CONCLUSIONS AND RECOMMENDATIONS .................................41

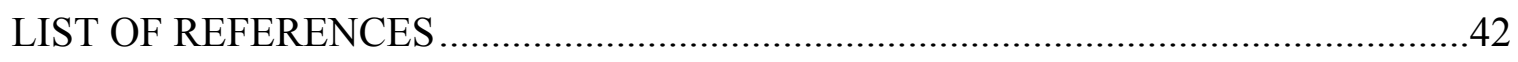

APPENDIX A: SPECIMEN ALIGNMENT GRIDS ...........................................47

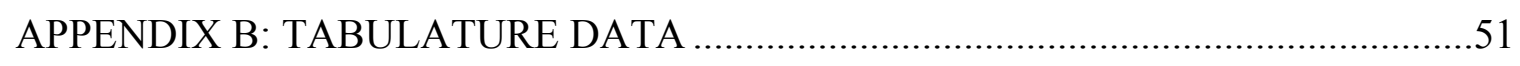

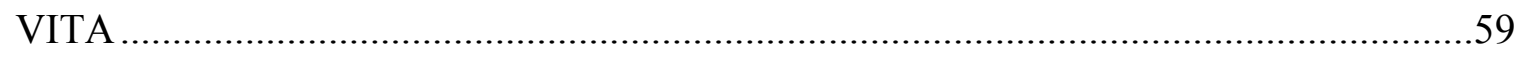




\section{LIST OF TABLES}

Table 5.1: Magnitudes and Angles of Resultant Forces for Flexion Testing .................. 38

Table 5.2: Magnitudes and Angles of Resultant Forces for Extension Testing................ 39

Table B.1: Harvested Spine Condition Flexion Data................................................... 51

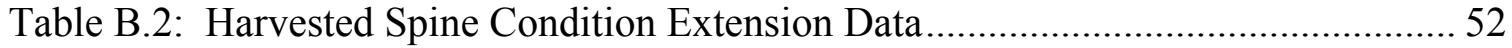

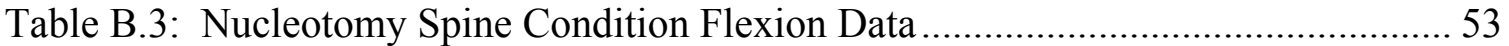

Table B.4: Nucleotomy Spine Condition Extension Data ........................................... 54

Table B.5: Compliant Implanted Spine Condition Flexion Data.................................. 55

Table B.6: Compliant Implanted Spine Condition Extension Data................................ 56

Table B.7: Non-Compliant Implanted Spine Condition Flexion Data .......................... 57

Table B.8: Non-Compliant Implanted Spine Condition Extension Data.......................... 58 


\section{LIST OF FIGURES}

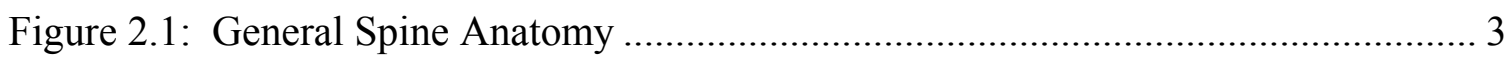

Figure 2.2: The Lumbar Spine ……………………….......................................... 3

Figure 2.3: Anatomy of the Intervertebral Disc........................................................... 5

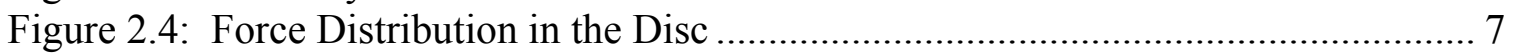

Figure 2.5: The Motion Segment Unit (MSU)................................................................ 7

Figure 2.6: Movement of the Motion Segment Unit......................................................... 8

Figure 3.1: Force Components of Disc ...................................................................... 14

Figure 3.2: Grid of Rotational Points...................................................................... 14

Figure 3.3: Potted Specimen in Robot …………………...................................... 16

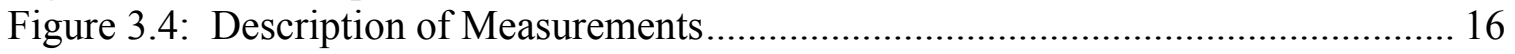

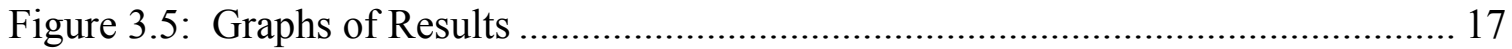

Figure 3.6: Flexion Resultant Force Vectors and Angles for Each Point of Rotation.... 19

Figure 3.7: Extension Resultant Force Vectors and Angles for Each Point of Rotation. 20

Figure 3.8: Translation Parallel to Disc Plane ……………………………………........ 22

Figure 4.1: Picture of Compliant and Non-Compliant Implants...................................... 27

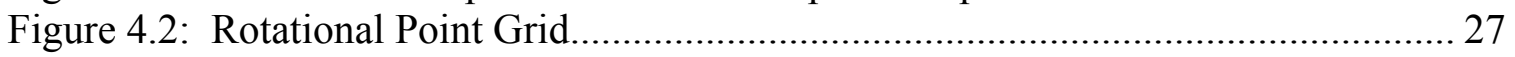

Figure 4.3: Explanation of Measurements ................................................................... 29

Figure 4.4: Graphs of Results, Including Non-Compliant Device.................................. 30

Figure 4.5: Flexion Resultant Force Vectors and Magnitudes, Including Non-

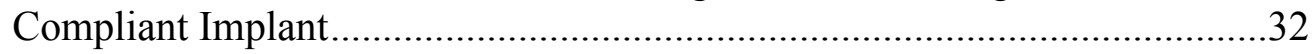

Figure 4.6: Extension Resultant Force Vectors and Magnitudes, Including Non-

Compliant Implant .........................................................................................

Figure 5.1: All Resultant Force Vectors and Angles ................................................... 37

Figure A.1: Grid for Specimen 0105416 L3-L4 ……………………...................... 47

Figure A.2: Grid for Specimen ADS0612051 L3-L4 ................................................ 47

Figure A.3: Grid for Specimen DRT050786 L3-L4 ……......................................... 48

Figure A.4: Grid for Specimen DRT060069 L3-L4 ……........................................... 48

Figure A.5: Grid for Specimen UF01A030 L3-L4 ................................................... 49

Figure A.6: Grid for Specimen UJ00D35 L3-L4 ....................................................... 49

Figure A.7: Grid for Specimen ADS0612051 L5-S1 ................................................. 50 


\section{CHAPTER 1: INTRODUCTION}

Over the past fifty years, significant progress has been made in the development of spinal implants. While spinal fusion remains the gold standard of surgical care, engineers and scientists continue to develop alternative surgical treatments. Today, patients with degenerative disc disease can elect to have total disc replacement surgery, a procedure aimed at maintaining or restoring segmental spinal motion, rather than just fusing the vertebrae together, which hinders global motion and may cause collateral damage to the surrounding tissues via adjacent segment disease. Another alternative surgical option under development is nucleus replacement. This process involves removing the nucleus pulposus and replacing it with either a mechanical or a compliant implant. Replacing just the nucleus has many benefits over removing the entire disc or fusion. First, most of the surgeries involved in implanting these devices are much less invasive and require a shorter healing time. Also, the surgeries can be performed in the early stages of disc degeneration. Performing the surgeries earlier allows for the retention of more healthy surrounding tissue. Several different models of nucleus replacement devices exist. Some involve using polymers and synthetic materials, or have rigid, mechanical properties. Others use a hydrogel core that mimics the swelling and shrinking characteristics of a normal nucleus pulposus. ${ }^{1 ; 18}$ The biomechanics of those that involve a hydrogel core are particularly interesting due to the compliant nature of the material used. Since these devices are designed to mimic some of the properties of the nucleus pulposus, the biomechanics and kinematics of the devices are much more difficult to describe. Currently, no testing protocol exists that measures the effectiveness of these compliant devices to function as the native tissue. ${ }^{24}$

The objective of this research was to evaluate different designs used in nucleus arthroplasty devices and to compare their restorative characteristics to the native tissue. A recently developed testing protocol was used that enabled simulation of different centers of rotation on the same motion segment unit (MSU) and comparison of the MSU mechanics for each spine condition. The main body of this thesis is divided into two separate manuscripts to be submitted for publication. 


\section{CHAPTER 2: BACKGROUND}

This chapter is organized into two main sections that serve as general background information for the two presented manuscripts. The first part of this chapter deals with the general anatomy of a spinal motion segment unit. It first describes the general structure of the spine, and then the structure and mechanics of the intervertebral discs. Finally, it describes exactly what defines a motion segment unit. The second part of this chapter describes degenerative disc disease and some of the therapies and surgical treatment options.

\section{Motion Segment Unit Anatomy}

\section{General Spine Anatomy}

The human spine consists of 32-34 vertebrae connected by intervertebral discs, various ligaments, and/or fusion. Of these vertebrae, seven are located in the cervical region, twelve in the thoracic region, five in the lumbar region, five fused in the sacrum, and three to five fused to form the coccyx. Figure 2.1 shows the basic shape of the spinal column. The kyphotic (concave anterior) and lordotic (convex anterior) curves of the spine shown in this figure help to further distribute weight.

The bony structures of the cervical, thoracic, and lumbar regions of the spine differ in size and shape, but contain the same basic structures. The largest section, or the body, distributes weight and provides the stability for the anterior section of the spine. The inferior and superior articular processes of adjacent vertebrae, along with the corresponding ligament, form the facet joint, which provides the main stability of the posterior section of the spine. The pedicles protrude from the main body and connect it to the posterior structures. These other bony structures include the foramen, spinous processes, and transverse processes.

While the spinal column distributes weight and allows for the upright stance of humans, it also provides other functions. The foramen protects the spinal cord and other nerve roots as they travel from the brain stem to the pelvis. The processes function as insertion points for both ligaments (to connect bony structures and provide stability) and tendons (to connect muscles for movement). The shape and design of the spinal column allows for range of motion in three anatomical planes: flexion-extension, axial rotation, and lateral bending.

In the lumbar region of the spine, seen in Figure 2.2, the vertebral bodies are significantly larger than the cervical and upper thoracic regions because it is responsible for much of the weight bearing and movement that occur during daily activities. The size of each body further increases from L1 through L5. As can be seen in Figure 2.1, the body is larger in the transverse direction and forms more of a bean shape. The height of the posterior section of the vertebral body is slightly less than the anterior sections in the 


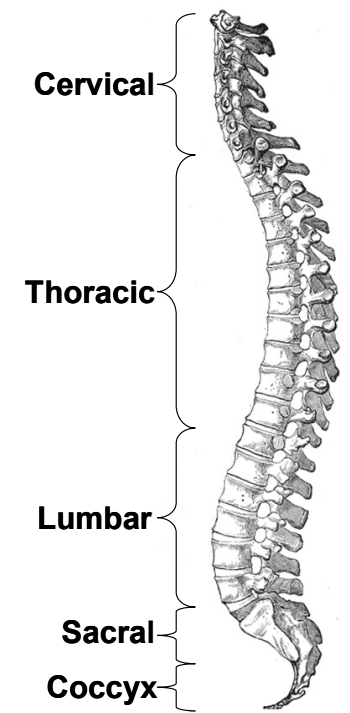

(A)

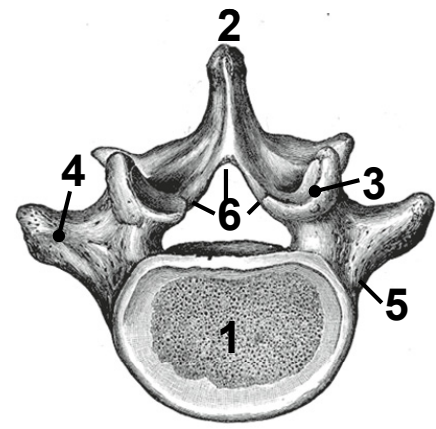

(B)

Figure 2.1: General Spine Anatomy. (A) Lateral view of the human spinal column. (B) The bony structures of the spine. The example shown is in the lumbar region. Each number denotes the major features, including the body (1) and processes $(2,3,4)$. The pedicle (5) provides stability and connects the body to the posterior elements. The neural arch, or foramen, (6) allows passage of the spinal cord. [Adapted with permission. Gray, Henry. Anatomy of the Human Body. Philadelphia: Lea \& Febiger, 1918; Bartleby.com, 2000. www.bartleby.com/107/illus111 and $94 .^{27}$ ]

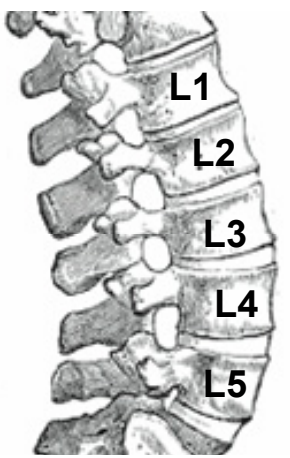

(A)

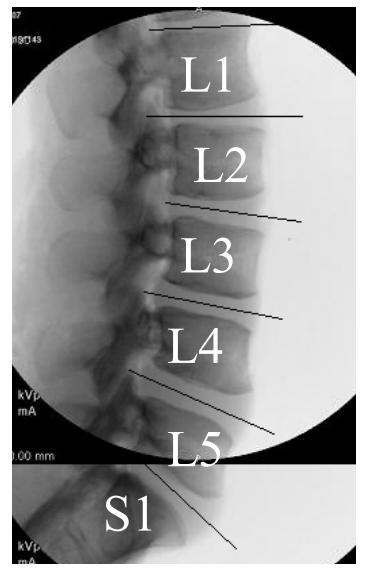

(B)

Figure 2.2: The Lumbar Spine. (A) Lateral view of the lumbar spine. [Adapted with permission. Gray, Henry. Anatomy of the Human Body. Philadelphia: Lea \& Febiger, 1918; Bartleby.com, 2000. www.bartleby.com/107/illus111. ${ }^{27}$ ] (B) Radiograph of lumbar spine showing angles of discs. The superior end of L1 is assumed to be horizontal. 
last three vertebrae, which influences the curve of the spine. ${ }^{15}$ The lordotic curve of the lumbar region also causes the L5-S junction to form a $30-45^{\circ}$ angle with the horizontal when the whole spine is positioned in a neutral upright orientation. Also when the spine is positioned in the neutral position, the cranial endplate of the L1 vertebral body is close to horizontal.

\section{Intervertebral Disc Anatomy and Mechanics}

From the second cervical vertebrae to the sacrum, the vertebral bodies are separated from each other by intervertebral discs. These discs serve to separate the bony sections of the main vertebral bodies. The compliant nature of the disc allows for more motion between the vertebrae. It also evenly distributes the weight across the entire endplate of the vertebral body and acts as a shock-absorber to prevent overloading of the main bodies. ${ }^{15}$

Each disc contains two main sections: the annulus fibrosus and nucleus pulposus. Figure 2.3 shows the estimated boundary between the two sections. This boundary is

distinct in children, but becomes more blurred in adulthood. ${ }^{15 ; 34}$ A third section, the cartilage end plates, is sometimes included in the structure of the disc because the cartilage of the endplates continues into the annulus. ${ }^{53}$ While both the annulus and the nucleus are composed of the same basic materials (collagen, proteoglycans, and water), the structures, make-up, and how these materials are arranged alter the functions of each of the sections.

The annulus consists of type I and type II cartilage arranged in fibrous, concentric rings, called lamellae. The collagen fibers attach at an angle to the endplates, then alternate direction in each subsequent layer. This structure allows the annular tissue to resist most of the tensile forces experienced in the disc. It also serves to connect the superior and inferior endplates in one, continuous structure.

The nucleus typically accounts for 40 percent of the cross-sectional area of the disc and is located centrally to slightly posteriorly in the disc space. ${ }^{15}$ The main components of the nucleus include type II collagen fibers surrounded by proteoglycans. The ratio of collagen to proteoglycans changes, depending on location in the disc and the position of the disc in the spine. Fewer proteoglycans exist towards the boundary between the nucleus and the annulus. This change gives the disc different viscoelastic properties depending on the types of loads it needs to bear. The presence of high concentrations of water allows the nucleus to resist the compressive forces of the disc. Because the proteoglycans attract water, the consistency of the nucleus is much more gellike than the annulus. The proteoglycans also aid in providing nutrients to the entire disc region. The disc itself does not contain any blood vessels except on the very outer layers of the annulus. It relies on nutrients that are pulled into the nucleus along with the water. As the disc is compressed, the waste products are pushed out along with excess water in a pumping mechanism. ${ }^{5}$ 


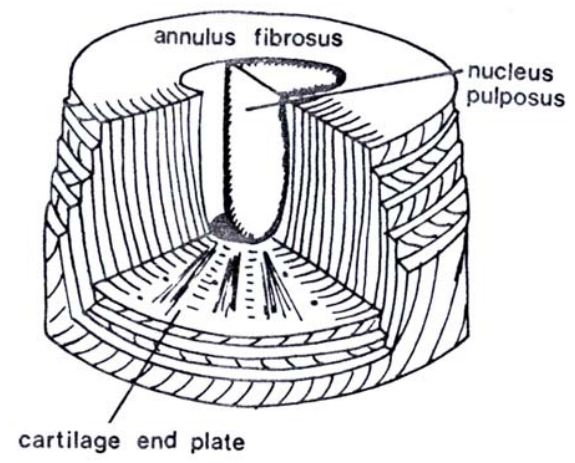

Figure 2.3: Anatomy of the Intervertebral Disc. [Reprinted with permission. Humzah MD and Soames RW. Human intervertebral disc: structure and function. The Anatomical Record 1988;220:337-56. ${ }^{34}$ ] 
Figure 2.4 shows the interaction between the nuclear and annular forces when a load is placed on the disc. Without any additional forces, the annulus is preloaded by the interaction with the hydrophilic nucleus. ${ }^{59}$ Because of these interactions, the entire disc acts as a pressurized chamber. As the disc is compressed uniformly, the nucleus pulposus distributes the force to the annulus in the form of tensile forces. ${ }^{28}$ As is shown in the figure, the annulus bulges outward, putting the majority of the fibers into tension. If the nucleus is damaged, the inner portions of the annulus can bulge inwards, ${ }^{45}$ changing the dynamics of the tissue. Also shown in the figure is how the forces on the spine change as it is put into flexion or extension. As the spine is put into flexion, anterior portions of the disc are put into compression while posterior portions are put into tension. The opposite loading mechanics occur in extension.

\section{Motion Segment Unit}

A motion segment unit, or MSU as used in in vitro testing, is a smaller unit of the entire spine. It is sometimes called a functional spine unit and can maintain all of the major motions of the intact spine. ${ }^{61}$ The major components of the MSU are show in Figure 2.5. These include two vertebral bodies, the intervertebral disc, and all relevant ligaments. Although muscle function is not replicated, as much soft tissue is preserved as possible to prevent disruption of pertinent structures.

As mentioned previously, the MSU maintains all the major motions of the intact spine. This fact is accomplished by maintaining the intervertebral joint. The intervertebral joint is actually a three-joint complex. The main joint is the intervertebral disc itself. The two other joints are the two posterior facet joints. The interactions of these three joints allow six degree of spinal movement, three translational and three rotational, shown in Figure 2.6. In reality, each movement of the spine in a plane is a coupled motion involving two translational and one rotational movement. The orientation of the facet joints in the lumbar spine, however, prevents some of the rotational movements. ${ }^{51}$ In the MSU, the lack of muscle involvement prevents the movements from being truly physiological. Certain in vitro testing conditions, however, may reasonably approximate very passive spine movements where muscle involvement is minimal.

\section{Disc Degeneration}

\section{Stages}

Degenerative disc disease (DDD) is a term used to describe both the natural and unnatural degeneration of the spinal discs. It is not clearly understood, or defined. It has been used to describe not just the degeneration of the disc itself, but also general degeneration of the spine, disc herniation, and back pain. ${ }^{54}$ The link between disc degeneration and low back pain has been shown, ${ }^{43}$ but it can also manifest with no visible symptoms. $^{11}$ 


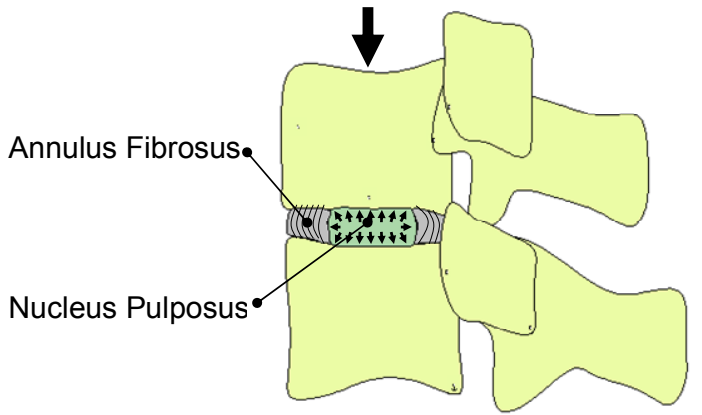

(A)

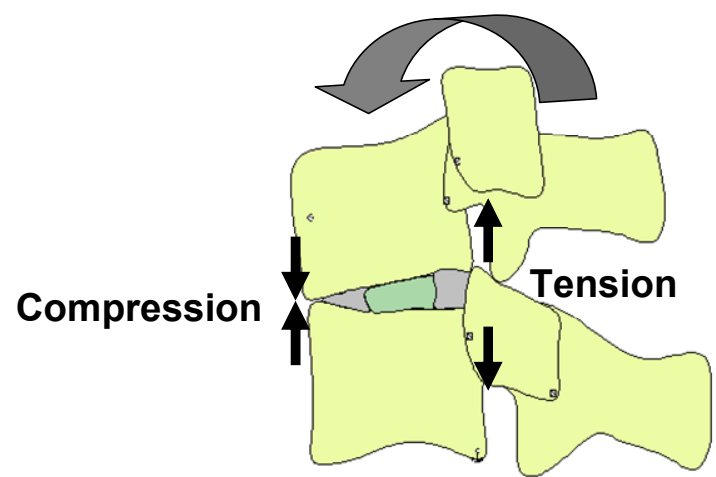

(B)

Figure 2.4: Force Distribution in the Disc. (A) As the disc is compressed by a force normal to the plane, the nucleus forces the annulus into tension. (B) Flexion or extension of the spine causes different areas of the disc to experience tension or compression.

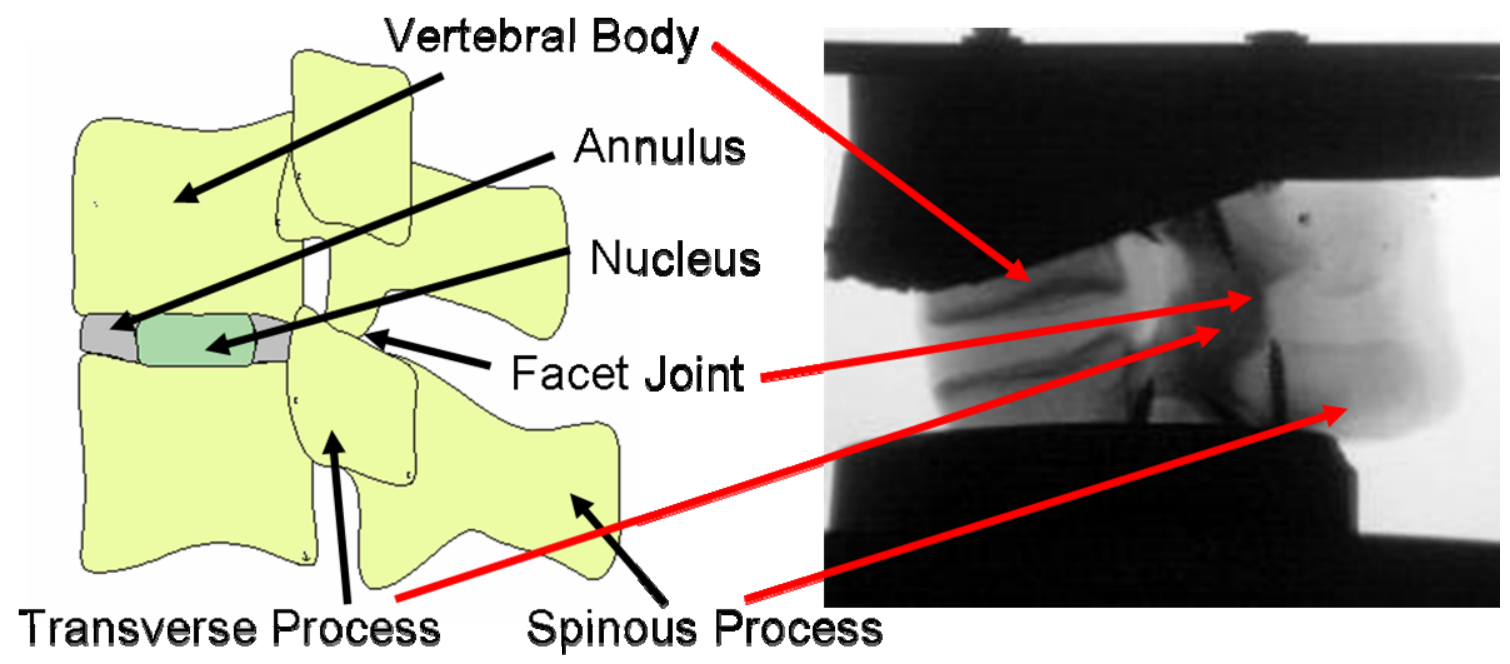

Figure 2.5: The Motion Segment Unit (MSU). The major bony features are labeled on both the drawing and the radiograph. 


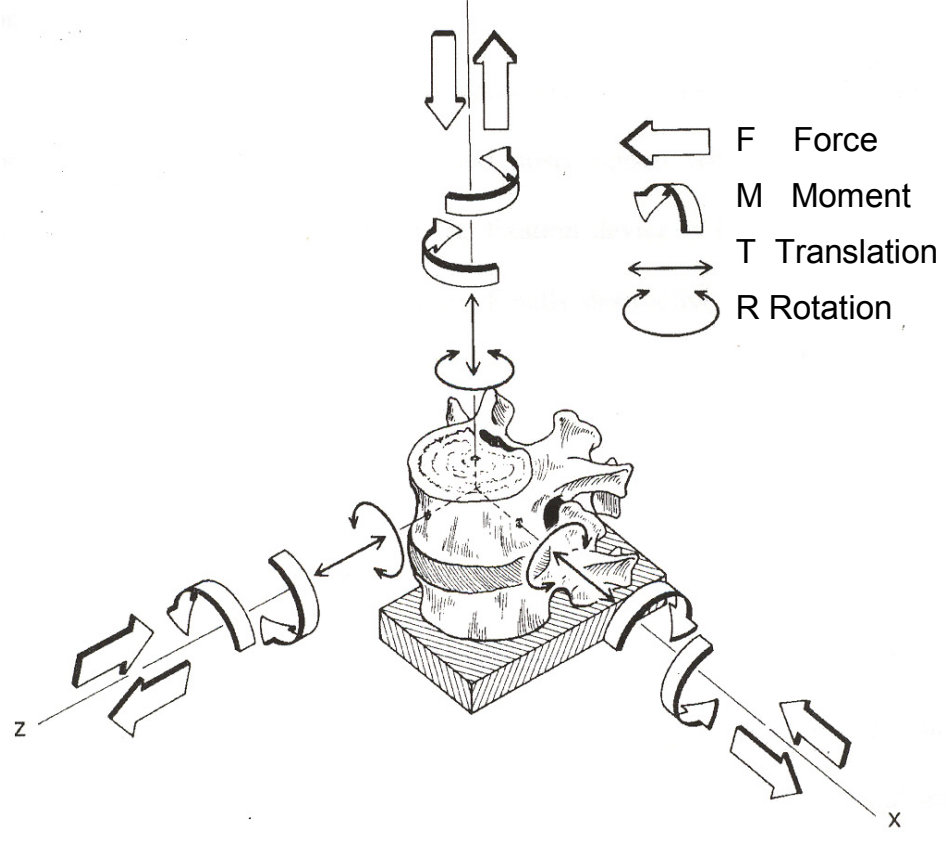

Figure 2.6: Movement of the Motion Segment Unit. The degrees of freedom of the MSU describe the motion. [Reprinted with permission. White AA and Panjabi MM. Clinical Biomechanics of the Spine. $2^{\text {nd }}$ ed. Philadelphia: J.B. Lippincott Co., 1990. pg $\left.45 .^{61}\right]$ 
Three stages of DDD are described by Kirkaldy-Willis and Farfan. ${ }^{36}$ While the boundaries of each stage are not specific, the amount of damage that occurs between the stages is significant. Stage I is also called the dysfunctional stage and begins in the $20 \mathrm{~s} .{ }^{1}$ It is also the least severe of the stages. The outer annulus begins to tear while the inner nucleus experiences changes in proteoglycan content. As the spinal column ages, the percentage of proteoglycans reduces, changing the water content of the nucleus to only $65-70 \%$. ${ }^{26}$ As the disc degenerates from its normal nucleus proteoglycan content, the more fibrous nucleus is less able to redistribute the forces it experiences to the outer annulus. Subsequently, the load the annulus experiences increases and the disc height decreases. Tearing of the outer annulus also leads to a disturbance of the natural pumping mechanism of the disc described earlier. The pain experienced during this stage can usually be treated, however, using conservative methods.

The instability stage, or stage II, occurs with further aging through the 30 s to the 50s. The amount of tissue damage, including delamination of the annular layers, becomes more severe and pronounced and leads to instability of the segment. The nucleus continues to change in proteoglycan content and water retention ability. Magnetic resonance images (MRIs) taken at this point reveal evidence of a "darker disc" and loss of disc height. ${ }^{1}$ The increased loads are also transferred to other components of the intervertebral joint, most notably the facets. ${ }^{21}$ Abnormal stresses may lead to further degeneration and breakdown of the joint. Treatment during this stage is more intense to combat the increased amount of pain.

Stage III, the stabilization stage, is the final stage of the degenerative process. It normally occurs when people reach the age of 60 and beyond. The ability of the disc to repair itself has been severely hampered by the lack of nutrients. Therefore, other repair mechanisms have taken over to attempt to stabilize the segment, including osteophyte formation and ligament thickening. The increased amount of tissue and decreased amount of disc space can lead to spinal stenosis. ${ }^{37}$ Spinal stenosis manifests as pain or numbness in the lower extremities due to the pinching or squeezing or the nerves from the lumbar spine.

\section{Treatment}

Most patients who suffer with DDD can be treated with conservative intervention with the main goal of easing pain. Pain medication, bed rest, and physical therapy are sufficient to manage the pain most people experience. Surgical intervention is sometimes required when conservative treatments for DDD fail to adequately remove the pain. A small segment $\left(1-2 \%^{1}\right)$ of the population requires surgical intervention. The most severe cases are recommended for fusion.

Davis et al. ${ }^{1}$ have applied the following criteria to determine if surgery is required for a particular patient:

1. Chronic low back pain of discogenic origin for more than six months that has failed a reasonable comprehensive nonoperative treatment program. This non- 
operative treatment program may include physical therapy, chiropractic manipulation, activity modification, a back education program, oral medications, and/or epidural spinal injections.

2. The absence of neurological signs and symptoms (radicular findings).

3. Evidence of abnormal disc morphology or DDD on MRI.

4. A concordantly positive provocative discogram which includes normal control levels above and/or below the degenerative disc in question.

Currently, three surgical treatments exist: partial discectomy, total disc replacement, or fusion. Early stage cases may undergo discectomy. Discectomy involves removing the disc material which is causing the pain. This may involve just removing part of the nucleus, all of the nucleus, or part of the annulus. In these cases, the pain may not be alleviated, ${ }^{4}$ and the condition may lead to an unstable condition. The degeneration of the remaining tissue is allowed to resume. In total disc replacement, an artificial disc is inserted in the disc space to replace the removed disc. Fusion is a more extreme surgery and involves fusing the two vertebral bodies of a MSU together. The fusion reduces the amount of motion that can occur at that level, which can alleviate the pain. It can also lead to an increase of stresses on the unfused segments ${ }^{41}$ and further degeneration. $^{42}$ 


\section{CHAPTER 3: BIOMECHANICAL PERFORMANCE OF A COMPLIANT NUCLEUS ARTHROPLASTY DEVICE}

\section{Introduction}

Degenerative disc disease (DDD) occurs in a wide segment of the population. Early studies showed prevalence of some stage of DDD in $96 \%$ of patients over the age of $60{ }^{40}$ More recent studies, however, show a wide variance in the amount of DDD in the adult population. ${ }^{7 ; 8}$ As the disease progresses, a loss of disc height occurs along with subsequent pain, increased load on the facet joints, and formation of osteophytes. ${ }^{5 ; 15 ; 46 ; 56}$ While fusion remains the gold standard of treatment for late stage DDD, the potential for early intervention and treatment of DDD has lead to the development of other therapies or treatments which retain many of the soft tissue elements and provide adequate motion. $1 ; 5 ; 6 ; ; ; 13 ; 14 ; 18 ; 29 ; 56 ; 59$ Recent advancements in biomaterial technologies have fostered growth in alternative surgical procedures to fusion surgery for treatment of early stages of DDD. One application of immediate interest which utilizes many of the recent advancements in biomaterial technologies is that of nucleus arthroplasty (NA) technologies. All of the different materials being developed can be classified as injectable devices, kinematically constrained mechanical devices, or load sharing devices. $^{1-4}$

Several reviews of nucleus replacement technology exist. ${ }^{6 ; 9 ; 18 ; 60}$ Most, however, focus on the history and development of the devices, but do not compare the different material selections within a specific implant design. One drawback to understanding emerging NA technologies is a lack of biomechanical studies in the literature.

Not only will the properties of the NA device affect the segmental mechanics, but so does the final placement. When considering a suitable material for a nucleus replacement device, each classification has a specific set of properties that are both advantageous and unfavorable to the restoration of the spine. One should understand the effects that material selection will have mechanical properties of the motion segment unit. In theory, the surgical steps of removing the nucleus, coupled with the nucleus replacement material, changes the work required to move the altered spine condition through a prescribed kinematics path relative to the intact spine condition. The closer the loading mechanics of the altered spine are to the intact spine condition, the more likely the device will restore the native properties.

The objective of this research was to study the lumbar segmental mechanics of a compliant NA device in vitro in a human cadaveric spine model and compare their restorative characteristics to the native tissue. A recently developed kinematics based testing protocol was used that enabled simulation of different centers of rotation on the same motion segment unit (MSU) and comparison of the MSU mechanics of the intact and altered spine conditions. 


\section{Materials and Methods}

\section{Testing Apparatus}

A multi-axis programmable spine robot $^{35}$ was used in this study that provided complete planar load and motion control of the spinal segment; conventional biomechanical testing systems including all of the multi-axis testing platforms currently being used in the spine research community cannot perform this task. ${ }^{10 ; 22 ; 4}$ The spine robot uses advanced user programmable control strategies that allow each axis to be independently programmed under displacement control, load control, or combinations thereof. Two translational and two rotational axes provide four degrees of freedom for complete control over sagittal plane movements. These unique features were used to generate the prescribed fixed motion paths used in this study. The two six axis load cells (JR3 Inc., Woodland, CA) recorded the forces and moments produced during the motions.

\section{Tissue Preparation}

Six fresh human cadaveric lumbar spines having an average age $30.3 \pm 14.8$ years were procured from the Medical Education and Research Institute (Memphis, TN). The spines were radiographically assessed by a spine surgeon using a fluoroscopic C-arm (GE 9600 or 9800, GE Healthcare, Chalfont St. Giles, United Kingdom) and were void of any gross tissue abnormalities. Each specimen was frozen prior to the tissue preparation and the potting phase. The spines were thawed for a 24 hour period and disarticulated into seven lumbar spinal MSUs (six x L3-L4 and one x L5-S). Each MSU included two vertebral bodies with the intact intervertebral disc, posterior bony structures, and connecting ligaments. Care was taken during dissection to maintain as much relevant soft tissue as possible. The bony end surfaces of the vertebral bodies were cleaned and mounted in either 4-inch cylindrical (L3, L4 and L5) or custom-designed sacral pots. The outer endplates of each spinal body was fixated into the pots using screws and a low melting point bismuth alloy (Small Parts, Miami Lakes, FL). The original disc angle and neutral position was measured using public domain software (Image J, National Institute of Health) and maintained during the potting procedure. Radiographic images were taken after the specimens were potted to verify disc angles: the average disc angles with respect to the superior endplate of L1 were $14^{\circ}$ for L3-L4 and $36^{\circ}$ for L5-S1.

\section{Surgical Spine Conditions}

Three separate surgical conditions were tested on each lumbar MSU: harvested, nucleotomy, and implanted spine conditions. The harvested spine condition had no surgical alterations after the specimen was potted and served as a baseline condition to determine the influence of the surgical procedures and the restorative effects of the implanted condition. For the nucleotomy and implanted spine conditions, a small incisional flap was made to the anterior aspect of the annulus. The flap provided access to perform the nucleotomy and insert the NA device. The HydraFlex ${ }^{\mathrm{TM}}$ Nucleus 
Arthroplasty System ${ }^{\mathrm{TM}}$ (Raymedica, Minneapolis, MN) was used for the compliant implanted spine condition. The device consisted of a hydrogel core and an ultra-high molecular weight polyethylene jacket. It was fully hydrated in a saline solution prior to implantation. The flap was sutured closed after the device was implanted.

\section{Test Protocol}

Although the conventional testing method of applying a pure or constant bending moment across a spinal construct and measuring the resulting motion response can be done, there are significant limitations with this testing methodology. Physiologically, the spine is not loaded with a constant bending moment, but rather experiences a moment distribution that varies across all spinal levels as you go down the spine. Further, a shear force component acts along the disc in vivo (Figure 3.1). Although pure moment methods provide a standard approach for comparing different lumbar spinal devices and may be acceptable for testing fusion instrumentation, ${ }^{25}$ they are not well suited for studying any type of spinal device that permits motion and/or has a variable stiffness or modulus, such as compliant NA or disc replacement devices. Also, studies that have used conventional pure moment testing methods cannot replicate the in vivo shear and axial force loading mechanics of the lumbar spine, which are inherent limitations. An alternative in vitro testing method is needed to biomechanically study compliant spinal devices.

A suitable testing protocol for NA must be able to distinguish between surgical conditions in terms of mechanics and kinematics. The natural progression of DDD and certain surgical treatments for DDD, including nucleotomy, may cause the spine to become unstable. White and Panjabi ${ }^{48 ; 61}$ have used sagittal plane translation greater than $4.5 \mathrm{~mm}$ or $15 \%$ of the anteroposterior diameter in a static radiographs as a sign of instability. Disch et al. ${ }^{19}$ used dynamic translations greater than $3.0 \mathrm{~mm}$ or $10 \%$ of the cranial vertebral body. A suitable testing protocol should include parameters that quantify these measurements relative to an in vivo measurement.

A new testing protocol was developed that prescribed a known kinematic input to a lumbar spinal MSU and measured the capacity of the intact MSU to accommodate the motion in terms of load response and range of motion. ${ }^{16}$ The kinematics path could be a simple rotation about a fixed point in space, or a coupled movement (displacement and rotation) along a path. The lumbar MSUs were mounted in the spine robot and tested first in flexion and then in extension. The MSUs were rotated about each point of rotation until a target bending moment of $8 \mathrm{Nm}$ of flexion or extension was reached. For all test conditions, MSU axial force normal to the disc plane, anterior-posterior shear force along the disc plane, sagittal rotation, and sagittal bending moment were measured.

\section{Simulation of Different Centers of Rotation}

For each specimen, a grid of points was identified on the radiographic images and their location determined using previously developed methodology ${ }^{12}$ (Figure 3.2). The 


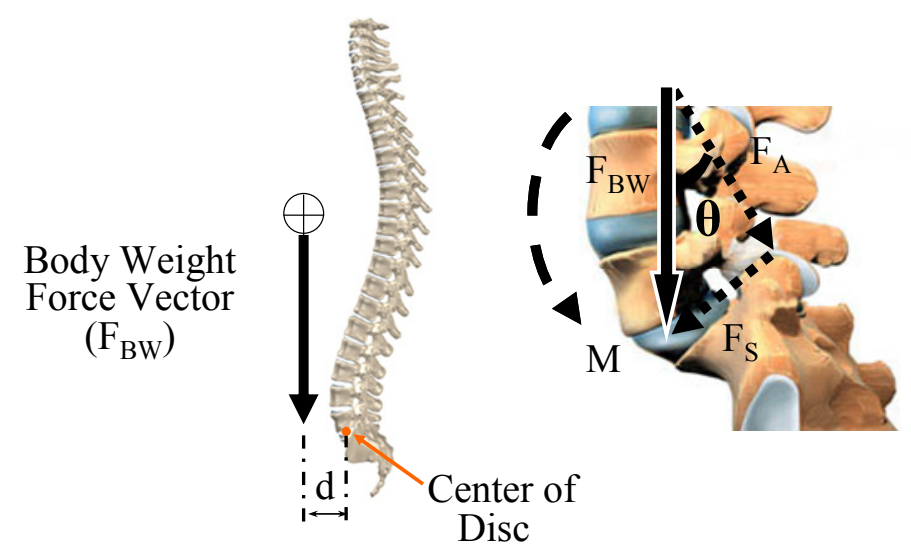

Figure 3.1: Force Components of Disc. The body weight force vector $\left(F_{B W}\right)$ induces a bending moment $\left(\mathrm{M}=\mathrm{F}_{\mathrm{BW}}{ }^{*} \mathrm{~d}\right)$. The shear component $\left(\mathrm{F}_{\mathrm{S}}\right)$ of the body weight acts along the plane of the disc. The axial component $\left(\mathrm{F}_{\mathrm{A}}\right)$ acts normal to the plane of the disc. As the spine flexes and extends, the moment arm changes, altering the axial and shear components.

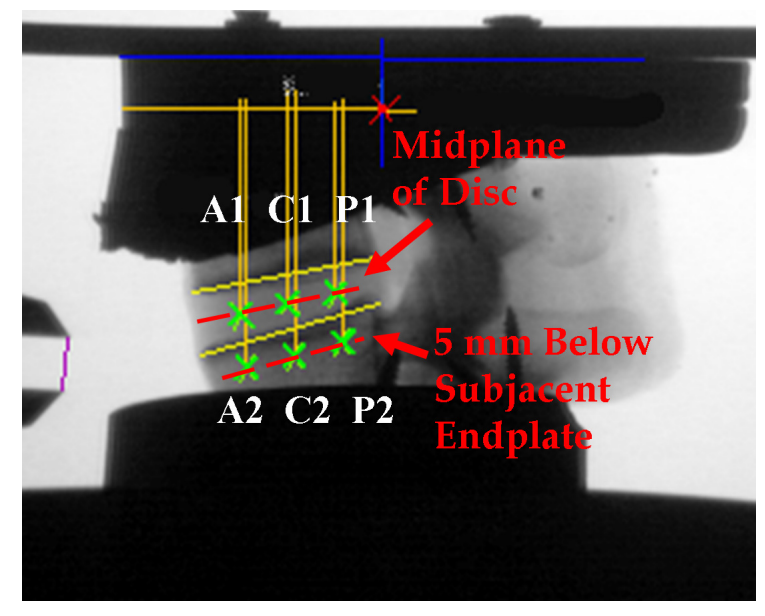

Figure 3.2: Grid of Rotational Points. A1, C1, and P1 lie along the midline of the disc. A2, C2, and P2 lie along the same orientation, but $5 \mathrm{~mm}$ below the endplate. 
fluoroscopic images were calibrated in Image $\mathrm{J}$ software and the following six points were located: 1) the mid point of the disc $(\mathrm{C} 1), 2)$ half way between the mid-point and anterior aspect of the disc (A1), 3) half way between the mid-point and posterior aspect of the disc (P1), 4) the same position as $\mathrm{C} 1$, but $5 \mathrm{~mm}$ below the endplate of subjacent vertebral body $(\mathrm{C} 2), 5)$ the same position as $\mathrm{A} 1$, but $5 \mathrm{~mm}$ below the endplate of subjacent vertebral body (A2), and 6) the same position as P1, but $5 \mathrm{~mm}$ below the endplate of subjacent vertebral body (P2). The horizontal (x) and vertical (z) distances of each rotational point in the grid relative to the Spine Robot's tool tip coordinate frame were recorded. The point coordinate data were programmed into the Spine Robot to establish the new rotational axes of each segment (Figure 3.3). Within the lumbar spine, some researchers place the center of rotation in the center of the disc, ${ }^{55}$ while others show the location more posteriorly. ${ }^{50 ; 52 ; 55 ; 57 ; 58}$ No consensus among researchers exists to where it lies during normal motion. By creating a grid of rotational points, this approach provided an indirect look at a variable center of rotation for each MSU.

\section{Measurements and Data Management}

An explanation of the conventions used in the test measurements is found in Figure 3.4. A positive rotation represents flexion, while a negative value represents extension. The axial and shear force components were recorded by the spine robot as a net force value acting on the MSU. For the axial force component, a positive value indicates a net tension and a negative value signifies a net compression. A positive shear force represents net posterior shear acting on the MSU and a negative shear force represents net anterior shear across the MSU. The amount of translation of the cranial vertebral body along the plane of the disc was also recorded. The mean values of the shear and axial forces, bending moments, and segmental rotations were statistically analyzed with SigmaStat (Systat Software, Inc). A ranked two-way repeated measures ANOVA and SNK test was used to determine significance $(p=0.05)$.

\section{Results}

The mean values of the range of rotation, axial force, and shear force data for all three spine conditions are shown in Figure 3.5.

\section{Harvested Spine Condition}

For the harvested condition, the range of rotation about the anterior points of rotation (points A1 and A2) were significantly less than the central or posterior points $(p<0.001)$. When comparing the axial force, a significant change in the type of force seen occurred between points of rotation during flexion testing. The net MSU axial force changed from tension to compression as the point of rotation moved from a mid or anterior point to a posterior point. This phenomenon was true for points along both planes (i.e., the disc plane and the subjacent body). As the rotation moved from an anterior point to a posterior point in both planes, the shear forces were directed anteriorly (negative recorded shear). 

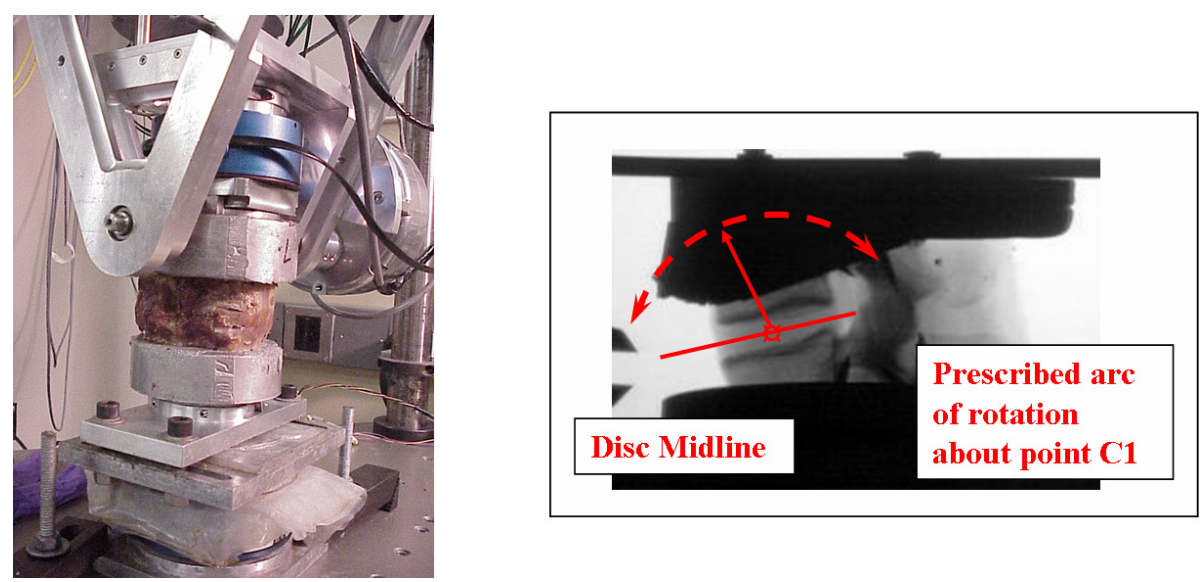

Figure 3.3: Potted Specimen in Robot. The picture to the right shows a potted MSU loaded in the Spine Robot. The figure on the left demonstrates how the transformed tool tip of the Spine Robot becomes the rotational axis for each of the grid points.

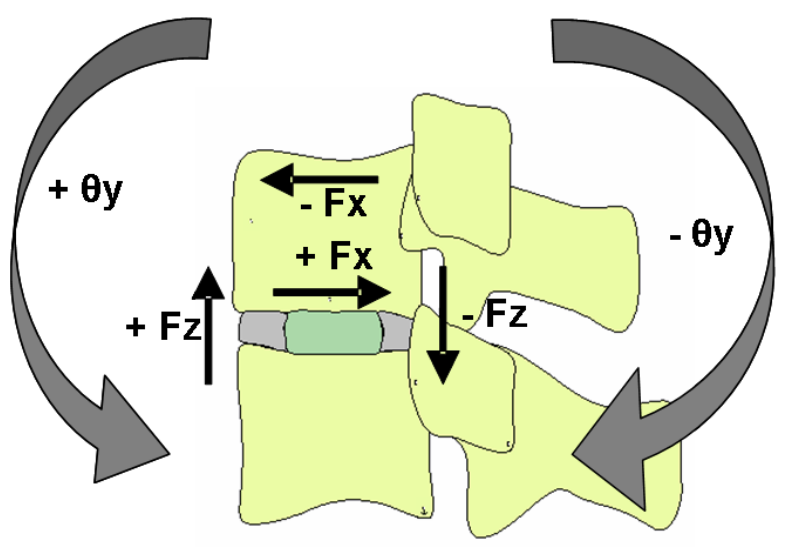

Figure 3.4: Description of Measurements. A positive rotation value $(+\theta y)$ indicates the MSU is in flexion, while a negative rotation value $(-\theta y)$ indicates extension. A positive axial force $(+\mathrm{Fz})$ indicates a net tissue tension, and a negative value $(-\mathrm{Fz})$ is net tissue compression. A positive shear force $(+\mathrm{Fx})$ indicates a net tissue shear towards the posterior structures, and a negative shear $(-\mathrm{Fx})$ is a net tissue shear towards the anterior structures. 

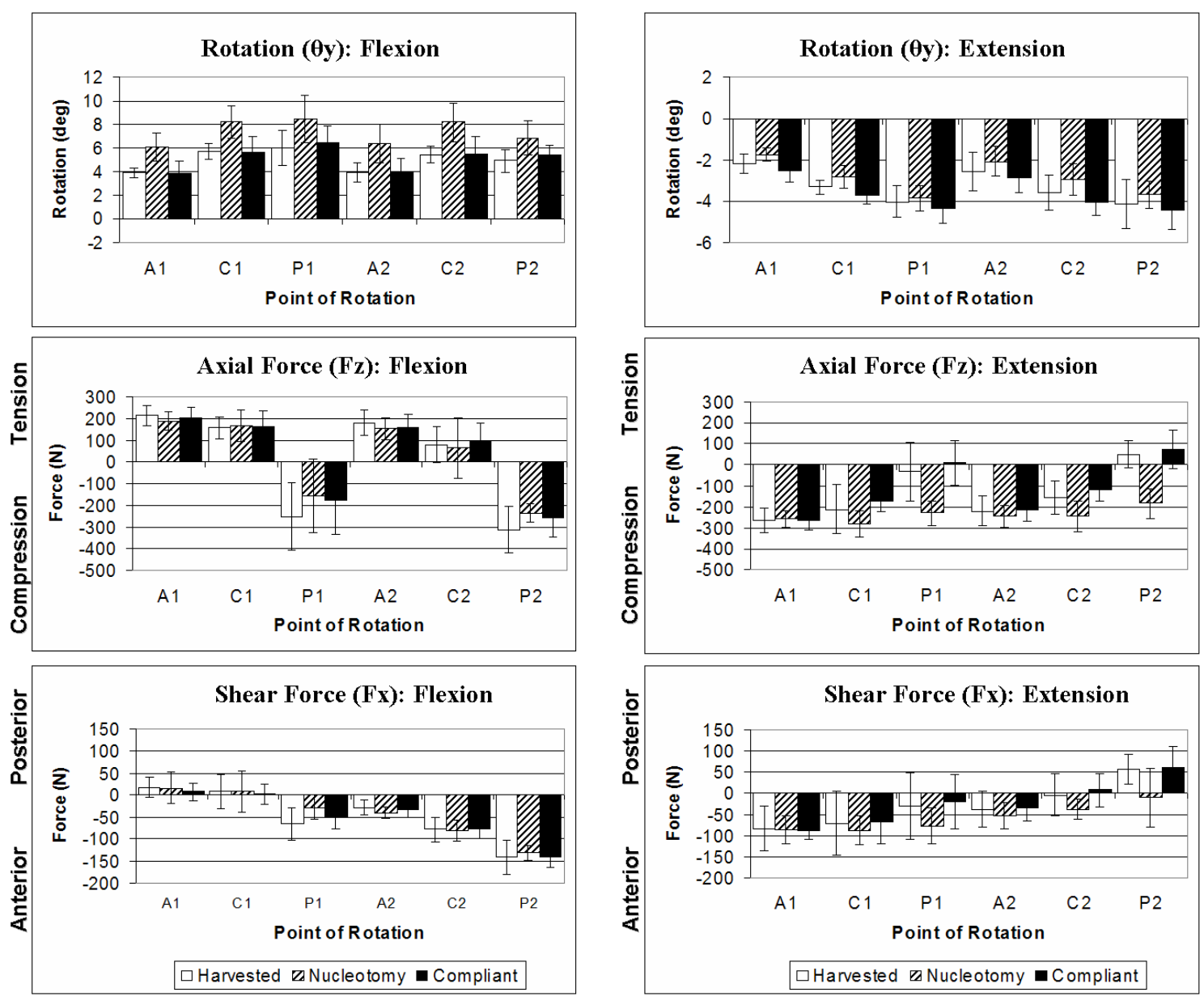

Figure 3.5: Graphs of Results. The top graphs show rotation in flexion (left) and extension (right). Note the greater rotation in the nucleotomy spine condition in flexion. The center graphs are net axial forces in flexion (left) and extension (right). There was a change from net tissue tension to net tissue extension in flexion as the point of rotation moved from anterior to posterior. The bottom graphs indicate net shear forces in flexion (left) and extension (right). 
In extension testing, rotation increased as the center of rotation moved more posteriorly in either plane. The net axial and shear forces acted in the opposite direction from flexion testing. As the center of rotation changed from an anterior point to a posterior point, the axial force became more tensile and the shear force directed towards the posterior aspects of the MSU (positive recorded shear).

\section{Nucleotomy Spine Condition}

The range of rotation in the nucleotomy condition was significantly greater than the other two spine conditions in flexion at all points of rotation $(\mathrm{p}<0.001)$. For example, the average rotation around the center of the disc (point $\mathrm{C} 1$ ) increased from $5.7^{\circ} \pm 0.7^{\circ}$ to $8.2^{\circ} \pm 1.4^{\circ}$ after the nucleotomy was performed. The forces on the spine also significantly changed during extension testing. Both the shear and compressive forces were significantly greater for the nucleotomy spine condition compared to the harvested and implanted spine conditions at the central and posterior rotational points $(\mathrm{C} 1, \mathrm{C} 2, \mathrm{P} 1, \mathrm{P} 2)$. The most posterior point along the plane of the disc (P1) showed a dramatic increase in both the amount of compression (harvested spine condition: $-30.3 \pm 138.8 \mathrm{~N}$; nucleotomy spine condition: $-230.0 \pm 59.9 \mathrm{~N}$; implanted spine condition: $-10.1 \pm 108.9 \mathrm{~N}$ ) and shear force (harvested spine condition: $-29.1 \pm 78.6 \mathrm{~N}$; nucleotomy spine condition: $-77.3 \pm 42.2$ $\mathrm{N}$; implanted spine condition: $-19.8 \pm 62.4 \mathrm{~N}$ ) when compared to the other two conditions.

\section{Implanted Spine Condition}

Implantation of the hydrated HydraFlex ${ }^{\mathrm{TM}}$ device returned upper limits of rotation and loading mechanics to an amount similar to the harvested spine condition for all points of rotation. There were no significant differences between the harvested spine condition and the implanted spine condition.

\section{Total Force Analysis}

The combined force vector (resultant of axial and shear force components) is shown in Figures 3.6 and 3.7 for the different spine conditions and points of rotation. The placement of the magnitude and position of the vectors on an arc instead of the center of rotation demonstrates which tissues are responsible for the resultant forces. For example, both the shear and axial components are positive at point A1 in flexion for all three spine conditions. The resultant forces indicate that the posterior tissue is responsible for tissue tension and posterior shear. Comparing point P1 in flexion, both the shear and axial force components become negative in all three conditions. The compression of the disc in the anterior portion of the MSU was dominant over posterior tensile forces seen at point A1. 

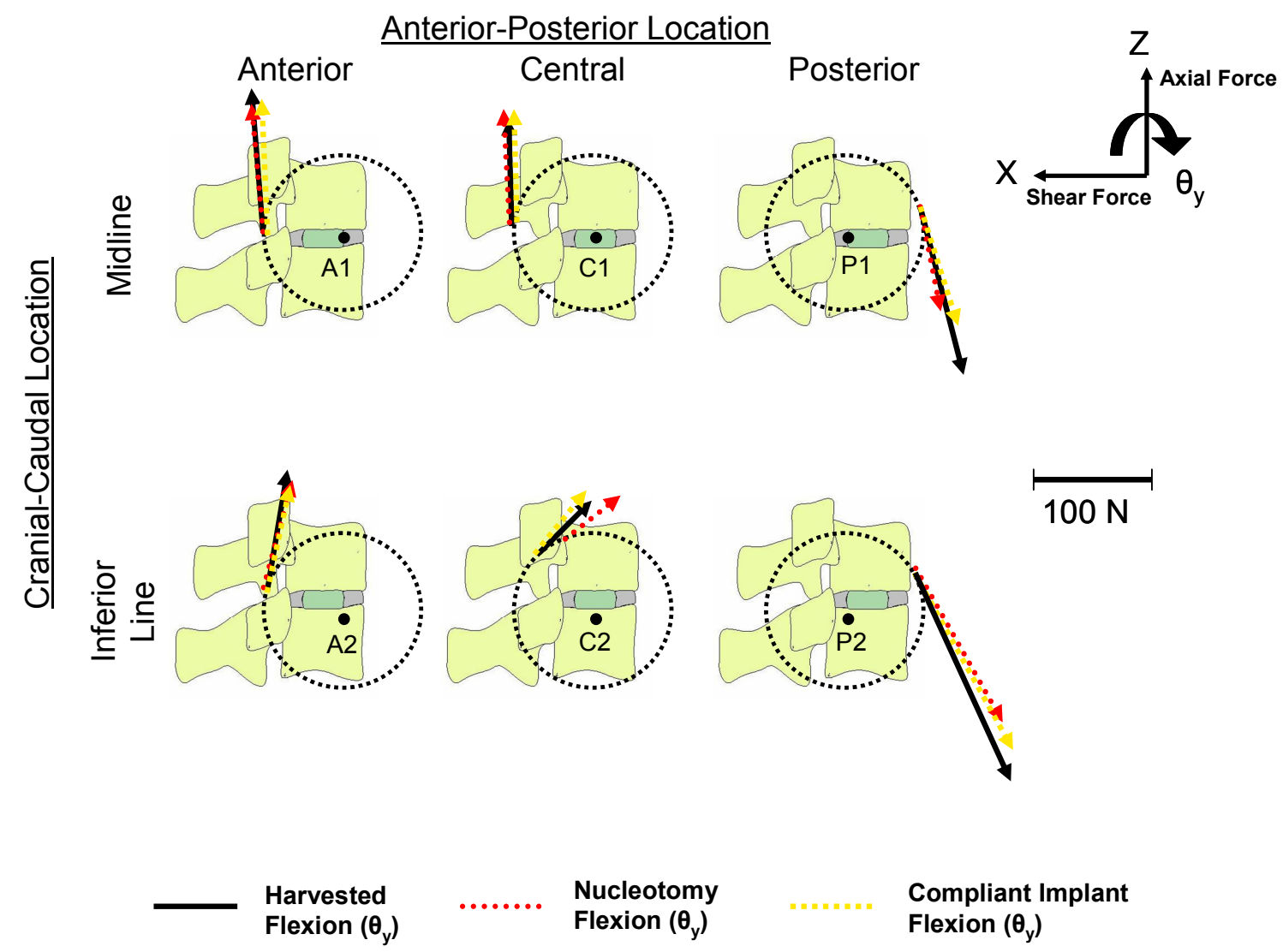

Figure 3.6: Flexion Resultant Force Vectors and Angles for Each Point of Rotation. The changes in tissue interactions from all three conditions can be garnered from the position and angle of the resultant vector. 


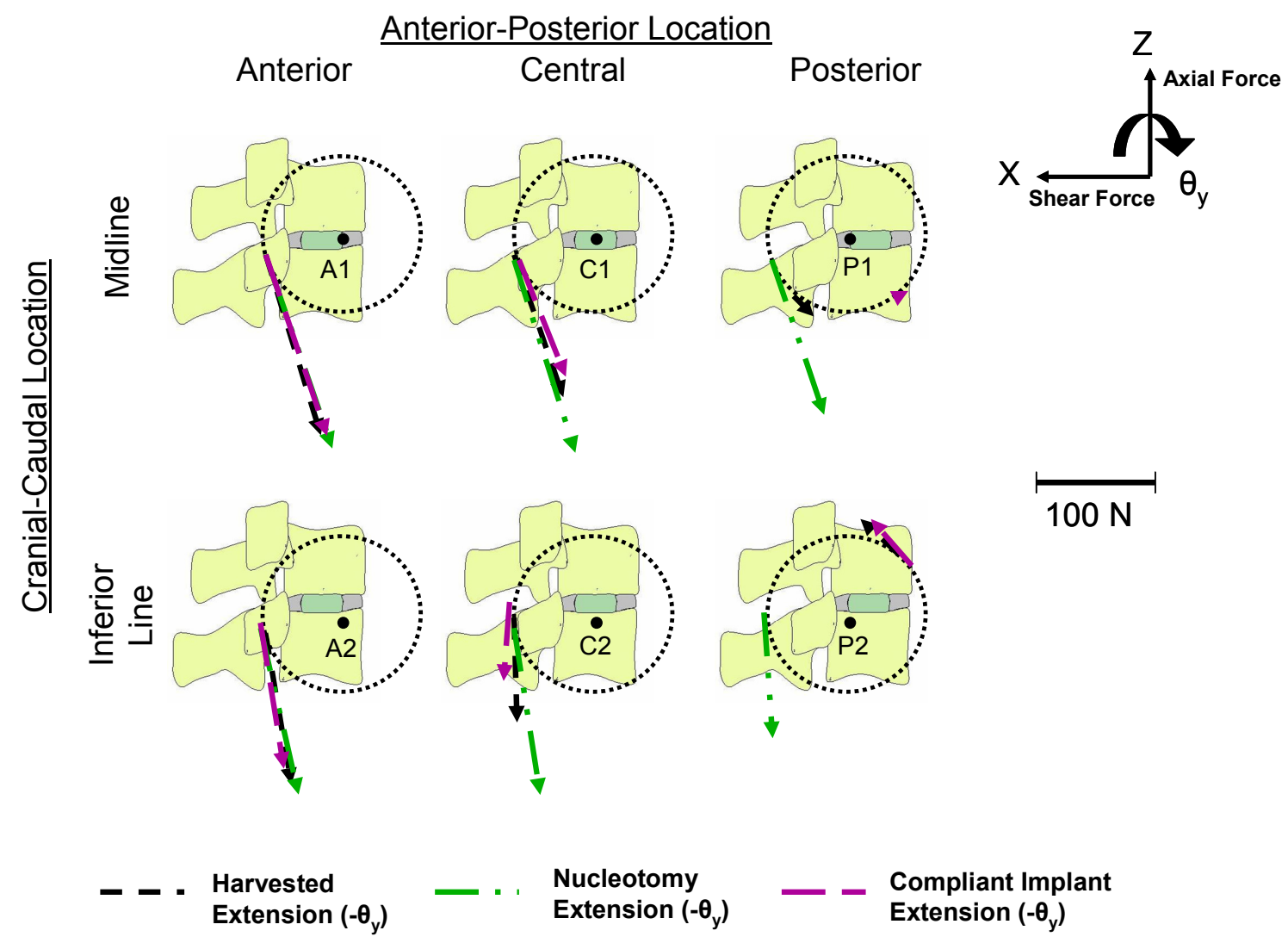

Figure 3.7: Extension Resultant Force Vectors and Angles for Each Point of Rotation. The changes in tissue interactions from all three conditions can be garnered from the position and angle of the resultant vector. 


\section{Translation along Plane of Disc}

The movement of the cranial vertebral body relative to the caudal body in flexion testing was transformed to the axis along the disc plane. This measurement is comparable to what is described as clinical instability. ${ }^{19 ; 61}$ For the three different spine conditions, the mean relative anterior translations calculated along the disc plane are presented graphically in Figure 3.8 for all points of rotation. For the nucleotomy spine condition, the segmental translation increased significantly for all points of rotation, except P1. The greatest translation occurred for the nucleotomy spine condition at points A2 $(2.9 \mathrm{~mm})$ and C2 $(3.0 \mathrm{~mm})$.

\section{Discussion}

Few biomechanical studies have been performed on denucleated spines. Using pure bending moments, Heuer et al. found the nucleotomy spine condition to be the most unstable condition for both range of motion and vertebral translation. ${ }^{30 ; 31}$ In a pure compression model, Meakin et al. saw a significant change in the manner that the disc deformed after a nucleotomy. ${ }^{45}$ However, the experiments they performed involved cutting the vertebral bodies and discs along the sagittal plane and attaching a window to monitor the deformations. As a consequence, they experienced problems with nucleus extrusion through the sealing of the viewing window. Frei et al. reported that the nucleotomy condition did not significantly affect functioning of the disc during shear loading of a functional spine unit. ${ }^{23}$ The shear load, however, was applied through the center of a horizontally mounted disc and was not combined with any other loading mode (i.e., no compression or rotation). As such, this loading case may have been idealized and less physiologically relevant. In the current study, maintenance of in vivo disc alignment and application of rotations about selected anatomical locations resulted in more physiologically relevant combined bending, axial, and shear loads. Denucleating the MSU led to a more destabilized condition with greater MSU rotation in flexion and greater disc compression in extension. It also significantly affected the amount of shear that the MSU experienced during both flexion and extension.

White and Panjabi developed a checklist of parameters, along with assigned point value system, to determine the presence of instability. ${ }^{61}$ The protocol used in this study involved direct measurements without using radiographic measurements. To apply the same definition as used from radiographs, a scaling factor of $25 \%$ must be incorporated to the original radiograph definition. ${ }^{61}$ Rescaling the definitions used by Disch et al. and by White and Panjabi to their absolute values results in a range of values between 2.2 and $3.3 \mathrm{~mm} .^{19 ; 61}$ In the current study, rotation around two points (A2 and C2) produced translations along the disc axis that exceeded this limit. While this measurement is only one aspect used to characterize clinical instability, it reinforces the idea that the nucleotomy spine condition represented an unstable condition.

After implantation of a hydrated HydraFlex ${ }^{\mathrm{TM}}$, variation in the kinematics were reduced and the response profile, including the rotation and loads, moved towards the 

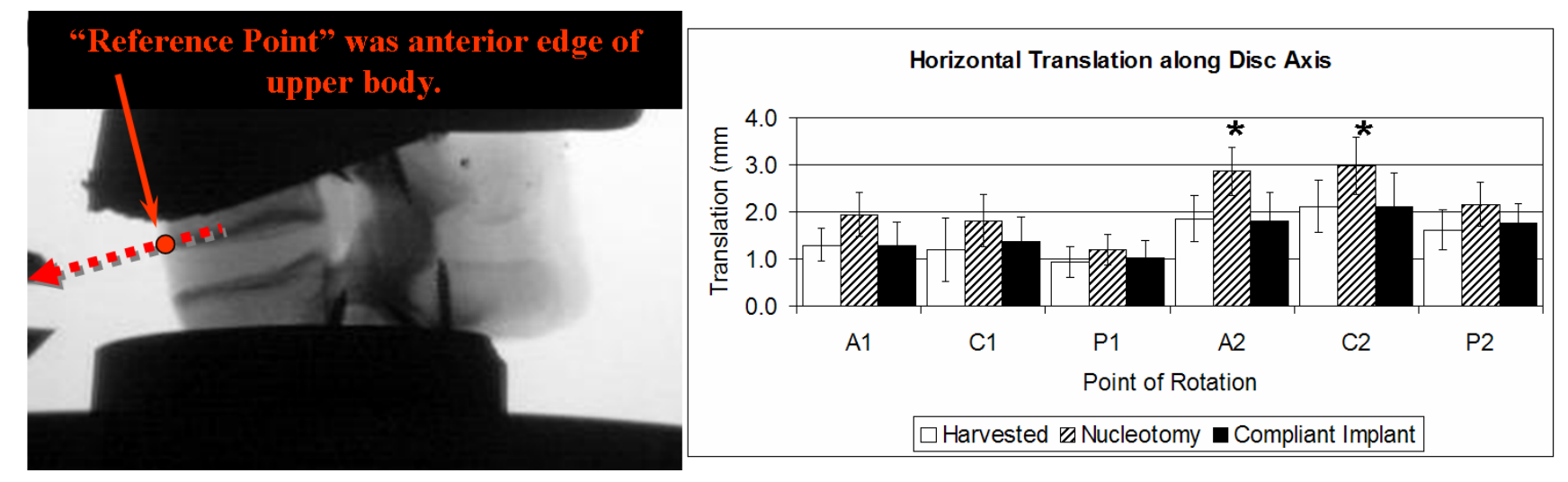

Figure 3.8: Translation Parallel to Disc Plane. The direction of the translation of the cranial vertebral body is shown in the figure on the left. The graph on the right shows the significantly greater translation for the nucleotomy condition for all points except P1. The * indicates where the definition of clinical instability was met. 
harvested state for all rotational points tested. Using finite element analysis, Meakin et al. ${ }^{44}$ predicted that implanting a material within the nucleus could prevent some of the changes in disc behavior.

While evaluating NA implants using MSUs can effectively replicate passive spinal movements, they cannot truly replicate physiological motions because of the lack of muscle involvement. Further investigation into different grid patterns may also help to successfully define centers of rotation to assess the different types of NA devices. The defined movements in this study were limited to the sagittal plane. Physiological motion of the spine involves coupled motions of both translational motion and axial rotation. All NA devices must also be evaluated using coupled motions before their restorative effects can be completely defined.

In this study, a spine robot was used to study the effects of changing the location of the MSU axis of rotation on the kinematic response of the harvested, nucleotomy, and implanted MSU. Since compliant nucleus replacement technologies do not have a prescribed axis of rotation, evaluating the kinematic response at multiple locations of rotation may more effectively characterize the restorative effect of these technologies compared to more traditional test methods. As new technologies evolve, the methods utilized to analyze their biomechanical performance must also evolve. Significant differences observed between the different center of rotations and spine conditions used in this study in terms of the range of rotation, compression, and shear response of the tissues indicated that the methodology used herein was of sufficient sensitivity to delineate between different nucleus conditions. 


\section{CHAPTER 4: COMPARISON OF COMPLIANT AND NON-COMPLIANT NUCLEUS ARTHROPLASTY DEVICES}

\section{Introduction}

Nucleus arthroplasty (NA) technologies are currently being developed as an alternative procedure to fusion surgery for treatment of degenerative disc disease (DDD). While most patients benefit from conservative treatments including physical therapy and pain medication, some patients' conditions are degraded enough to warrant surgical intervention. Fusion surgery remains the gold standard for surgical treatment for DDD, however total disc replacement and NA technologies are being increasingly researched and trialed as alternative treatments. In mild and early DDD cases, NA may have some advantage since native tissue is retained and further degenerative changes to native tissues may be potentially delayed.

Several authors have reviewed the current technology for nucleus replacement. ${ }^{5 ; 6 ; 9 ; 18 ; 29 ; 60}$ Most articles focus on the different materials being researched and the different types of implants. But a universal classification of the various types of nucleus replacement devices does not exist within the reviews. Davis et al. classify NA devices several different ways in their Nucleus Arthroplasty Technology series. The most common classification used is hydrogels, polymers/synthetics, and mechanical devices. ${ }^{1 ; 3 ; 4}$ In Volume II, the classifications have changed slightly to mechanical, polymer, and tissue engineered devices. ${ }^{2}$ The polymer devices are further divided into preformed and in situ formed. Preformed and in situ formed can also be used classify all NA devices. ${ }^{3 ; 4}$ Bertagnoli, et al. used the following classifications: constrained and/or predefined geometry, unconstrained devices, and injectable devices. ${ }^{9}$ However, this classification does not differentiate between compliant and non-compliant devices. Perhaps a better classification is as follows: void fillers (injectable), kinematically constrained mechanical devices (non-compliant), and load sharing devices (compliant). This classification describes the type of device and its main biomechanical function. Each of these materials, regardless of the classification, has material and mechanical properties that add to, or detract from, the ability to restore the native spine movements. In vitro testing protocols that can assess and quantify these properties in a physiologically relevant manner are essential to the development and approval of any device.

The objective of this study was to use a fixed center of rotation (COR) kinematics based protocol to compare the biomechanical properties of non-compliant and compliant nucleus arthroplasty devices in a human cadaveric lumbar spine model. This testing protocol enables simulation of different centers of rotation on the same motion segment unit (MSU) and comparison of the MSU mechanics between different spine conditions. 


\section{Materials and Methods}

\section{Testing Apparatus}

A custom built, multi-axis programmable robot $^{35}$ that attains complete kinematic control of the MSU was used instead of conventional biomechanical testing systems. ${ }^{10 ; 22 ; 24}$ The system combines two translational and two rotational axes with two six axis load cells (JR3 Inc., Woodland, CA) into a universally programmable spine testing system. The robotic controller (Adept Technologies, Livermore, CA) is user programmable and completely controls sagittal plane movements. Because each axis of the spine robot can be programmed under displacement control, load control, or a combination, it is better suited to study the differences between compliant and noncompliant devices.

\section{Tissue Preparation}

Six fresh cadaveric lumbar spines with an average age $30.3 \pm 14.8$ years were acquired from the Medical Education and Research Institute (Memphis, TN). None of the causes of death were due to injury or disease of the spine. Radiographic images of the specimens were taken using a fluoroscopic C-arm (GE 9600 or 9800, GE Healthcare, Chalfont St. Giles, United Kingdom). Each image was examined by a spine surgeon to evaluate specimen condition. The specimens were frozen immediately after harvest and thawed 24 hours before disarticulation into six x L3-L4 and one x L5-S lumbar spinal MSUs. During dissections, the tissue was again visually inspected for quality, with special attention given to the discs of interest. Each MSU included two vertebral bodies with the intact intervertebral disc, posterior bony structures, connecting ligaments, and as much relevant soft tissue as could be maintained. The bony end surfaces of the vertebral bodies were cleaned and mounted in either 4-inch cylindrical pots (L3, L4 and L5) or custom sacral pots. Each specimen was fixated into the pots using screws and a low melting point bismuth alloy (Small Parts, Miami Lakes, FL), preserving the neutral position and original disc angles (measured radiographically using public domain software Image $\mathbf{J}$ from the National Institute of Health). Radiographic images were taken of potted specimens to verify disc angles (average disc angles with respect to the superior endplate of L1: L3-L4 $\rightarrow 14^{\circ}$; L5-S1 $\rightarrow 36^{\circ}$ ).

\section{Surgical Spine Conditions}

The human lumbar MSUs were tested in four surgical conditions. These conditions included the harvested, nucleotomy, compliant implanted, and non-compliant implanted spine conditions. The harvested spine condition served as a baseline comparison for the other conditions and had no surgical alterations after the potting procedure. The nucleotomy was performed through a small anterior flap in the annulus. The HydraFlex ${ }^{\mathrm{TM}}$ Nucleus Arthroplasty System ${ }^{\mathrm{TM}}$ (Raymedica, Minneapolis, MN) was used for the compliant implanted spine condition and implanted through the same annular flap. The device consists of a hydrogel core and an ultra-high molecular weight 
polyethylene jacket. It was fully hydrated in a saline solution prior to implantation. The flap was sutured closed after the device was implanted. After testing, the compliant implant was removed and an aluminum replica of the hydrated HydraFlex ${ }^{\mathrm{TM}}$ was inserted. The size and shape of the non-compliant, metallic device was comparable to that of the hydrated HydraFlex ${ }^{\mathrm{TM}}$ implant under a $100 \mathrm{~N}$ load (Figure 4.1). Previous research noted the differences between the harvested, nucleotomy, and compliant implanted spine conditions (see Chapter 3). The data of this section focuses on comparing the non-compliant implant spine condition to the other three conditions.

\section{Testing Protocol}

There have been few biomechanical studies to date that have evaluated the different NA devices. Few biomechanical testing protocols have the ability to adequately characterize the restorative effects of a compliant device. Protocols which are normally used to evaluate fusion devices, including flexibility and stiffness protocols, ${ }^{47}$ cannot effectively describe the kinematics of non-fusion devices. ${ }^{49}$ Mechanical, or noncompliant, devices typically have a prescribed axis of rotation, whereas load sharing, or compliant, devices do not. The differences in the position of the axis of rotation can have a profound effect on how the device and the tissue behave. The alignment of the instantaneous axis of rotation (IAR) of a spinal device with the native spinal segment's IAR is important for optimal performance of motion preservation devices. Misalignment of the device IAR with the native IAR can lead to an overconstrained condition. ${ }^{17}$ There is currently no consensus, however, of where the IAR of the lumbar disc is located during flexion/extension movements. ${ }^{50 ; 52 ; 55 ; 57 ; 58}$ Therefore, to compare all the different types of NA devices (void fillers, mechanical, and load sharing), a different testing methodology must be used.

As mentioned previously, few biomechanical tests exist that can adequately characterize the effects of NA devices. To see the changes that occur to the native spine when a device of any material is introduced, the movements and responses of the native spine must first be characterized. The proposed approach herein was to test using different points of rotation to account for the lack of a defined center of rotation. The testing protocol used in this study involved a grid of six points which approximated the different regions within the spinal MSU where the IAR has been previously reported to be located. ${ }^{50 ; 52 ; 55 ; 57 ; 58}$ The spinal segments were mounted in the spine robot and tested first in flexion and then in extension. The MSUs were rotated about each of the six points of rotation until a target bending moment of $8 \mathrm{Nm}$ of flexion or extension was reached. For all test conditions, MSU axial force, anterior-posterior shear force, sagittal rotation, and sagittal bending moment were measured.

\section{Simulation of Different Centers of Rotation}

The grid of testing points ${ }^{12}$ was determined from the calibrated radiographic images of the potted specimens (Figure 4.2). The spine robot used these points as a CORs. The horizontal (x) and vertical (z) distances from the robot's wrist axis location 

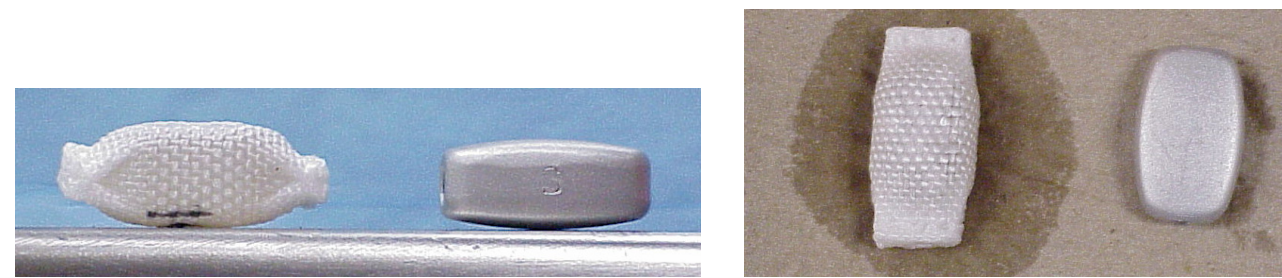

Figure 4.1: Picture of Compliant and Non-Compliant Implants. As can be seen the in photos, the aluminum non-compliant implant is roughly the same shape and height of the hydrated, compliant implant.

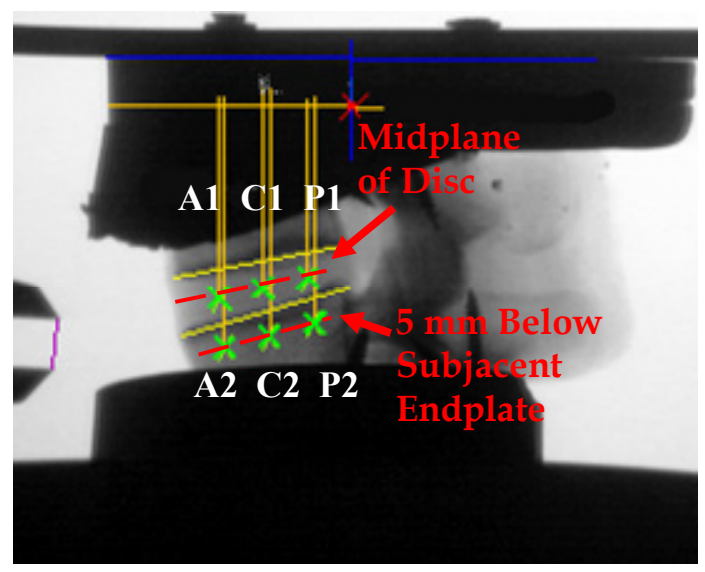

Figure 4.2: Rotational Point Grid. A1, C1, and P1 lie along the midline of the disc. $\mathrm{A} 2, \mathrm{C} 2$, and P2 lie along the same orientation, but $5 \mathrm{~mm}$ below the endplate. 
were programmed for each point in the selected grid into the controller. The COR was then transformed by the spine robot to the six programmed points. The grid consisted of three points along the center of the disc at the mid point (C1), half way between the midpoint and anterior aspect of the disc (A1), and half way between the mid-point and posterior aspect of the disc (P1), and three points located in the same anterior-posterior location, but $5 \mathrm{~mm}$ below the endplate of the subjacent body (A2, C2, and P2).

\section{Measurements and Data Management}

An explanation of the conventions used in the test measurements is found in Figure 4.3. In rotation, a positive value indicated flexion, while a negative value indicated extension. The force values (axial and shear) are recorded by the robot as a net force. For axial force, a positive value indicates a net tensile force and a negative value shows a net compressive force. A positive value in shear is a net shear force towards the posterior elements of the MSU and a negative value is a net shear force towards the anterior elements. The amount of translation of the cranial vertebral body along the plane of the disc was also recorded. The averages and standard deviations between the specimens for each testing measurement were compiled and compared using SigmaStat (Systat Software, Inc). A ranked two-way repeated measures ANOVA and SNK test was used to determine significance $(\mathrm{p}=0.05)$.

\section{Results}

The average values of the range of rotation, axial force, and shear force data for all four spine conditions are shown in Figure 4.4. The following results focus on the harvested, compliant implanted, and non-compliant implanted spine conditions.

\section{Harvested Spine Condition}

For the harvested condition in flexion testing, the range of rotation increased as the point of rotation moved from an anterior COR to a posterior COR along the plane of the disc. The net MSU axial force also changed to a more compressive force as the point of rotation moved from an anterior COR to a posterior COR along both planes (disc plane and subjacent body) during flexion motion. There were no significant differences between the points, however. The same is true for shear force in flexion. The shear force moved to a more anterior (negative) shear as the COR moved posteriorly. In extension testing, the opposite trends occurred in forces (more tensile force and anterior shear as the COR moved posteriorly), but the range of rotation still increased.

\section{Compliant Implanted Spine Condition}

The following results concentrate on comparing the compliant implanted spine condition to the harvested and nucleotomy spine conditions. The nucleotomy spine condition was significantly different from the compliant implanted spine condition in 


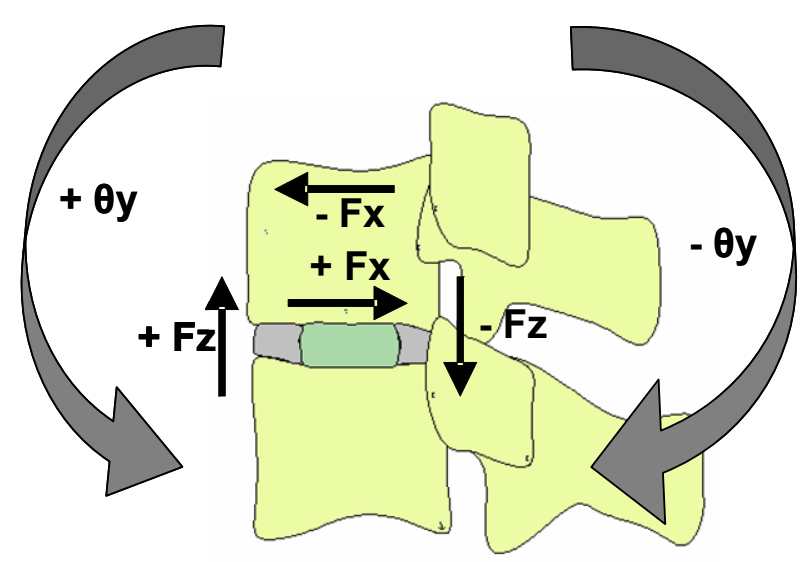

Figure 4.3: Explanation of Measurements. A positive rotation value ( $+\theta y)$ indicates the MSU is in flexion, while a negative rotation value $(-\theta y)$ indicates extension. A positive axial force $(+\mathrm{Fz})$ indicates a net tissue tension, and a negative value $(-\mathrm{Fz})$ is net tissue compression. A positive shear force $(+\mathrm{Fx})$ indicates a net tissue shear towards the posterior structures, and a negative shear $(-\mathrm{Fx})$ is a net tissue shear towards the anterior structures. 

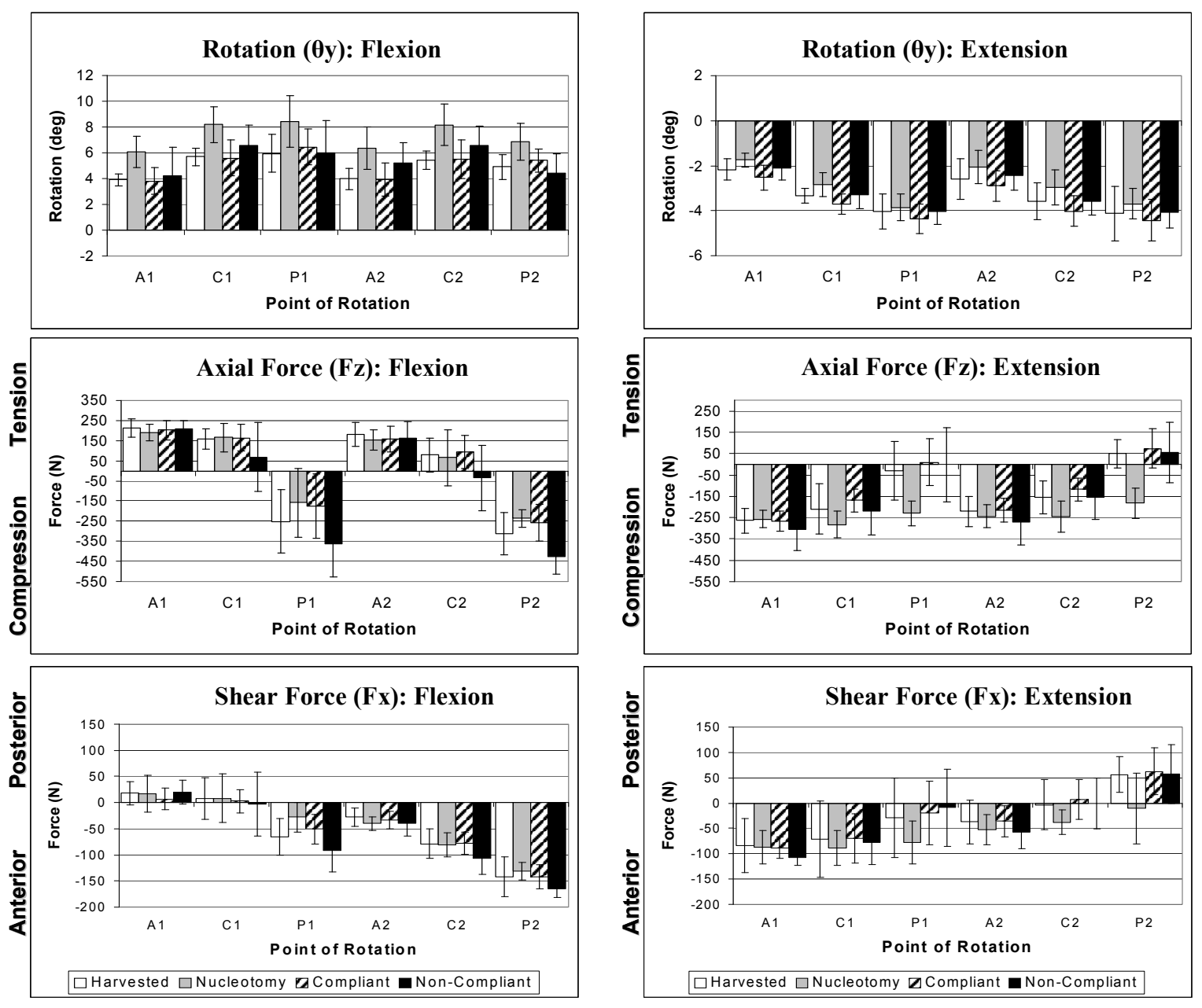

Figure 4.4: Graphs of Results, Including Non-Compliant Device. The top graphs show rotation in flexion (left) and extension (right). The center graphs are net axial forces in flexion (left) and extension (right). The bottom graphs indicate net shear forces in flexion (left) and extension (right). Note the increased amount of compression and shear in flexion for the non-compliant implant at posterior points. 
several measurements. In flexion testing, it caused more rotation around all points of rotation $(\mathrm{p}<0.001)$. In extension testing, it increased the amount of compressive and shear force at the central and posterior points $(\mathrm{C} 1, \mathrm{C} 2, \mathrm{P} 1, \mathrm{P} 2)$. There were no significant differences, however, between the harvested spine condition and the compliant implanted spine condition in any measurement at any point of rotation.

\section{Non-Compliant Implanted Spine Condition}

Similar to the compliant implanted spine condition, the non-compliant implanted spine condition was significantly different from the nucleotomy spine condition. When compared to the harvested or compliant implanted spine conditions, however, the differences were noteworthy. The non-compliant implanted spine condition had more of an effect on the axial forces in flexion than it did for any other measurement. At points $\mathrm{P} 1, \mathrm{P} 2$, and $\mathrm{C} 2$, the non-compliant implanted spine condition produced significantly greater compressive forces than any other spine condition. This result was especially noticeable at point P2. The compressive force for the harvested case was $-313.0 \pm 106.3$ $\mathrm{N}$. The compressive force for the compliant implanted case was $-257.2 \pm 91.2 \mathrm{~N}$. The non-compliant implanted spine condition, however, significantly increased the amount of compressive force to $-425.5 \pm 86.0 \mathrm{~N}$. The anterior shear forces were significantly greater for the non-compliant implanted spine condition compared to the other spine conditions during flexion testing at point P1. Harvested shear force at point P1 was $-65.4 \pm 35.6 \mathrm{~N}$. The compliant implanted spine condition decreased slightly, but not significantly, to $-50.5 \pm 28.2 \mathrm{~N}$. The non-compliant implanted spine condition increased significantly over both the harvested and compliant implanted spine conditions to $-92.2 \pm 40.0 \mathrm{~N}$. The rotations between the non-compliant implanted and the compliant implanted spine conditions were not significantly different.

\section{Total Force Analysis}

The resultant force vectors (combined axial and shear forces) for flexion rotation are shown in Figure 4.5. Even though the resultant force vector acts through the COR, the placement on an arc surrounding the COR better illustrates the tissues responsible for the combined forces experienced within MSU during motion. The differences between the spine conditions can be clearly determined, especially between the non-compliant implanted spine condition and the other spine conditions at central and posterior points of rotation. For example, at points $\mathrm{P} 1$ and $\mathrm{P} 2$, the magnitude of the force vector is much greater than the other spine conditions, indicating that the compressive forces are much greater in the anterior portion of the disc. Figure 4.6 shows the same information, but for extension motion.

\section{Discussion}

The results of this study demonstrated few significant differences between the compliant and non-compliant implant devices. The differences that did occur, however, tended to involve rotation about central and posterior points. The most common 


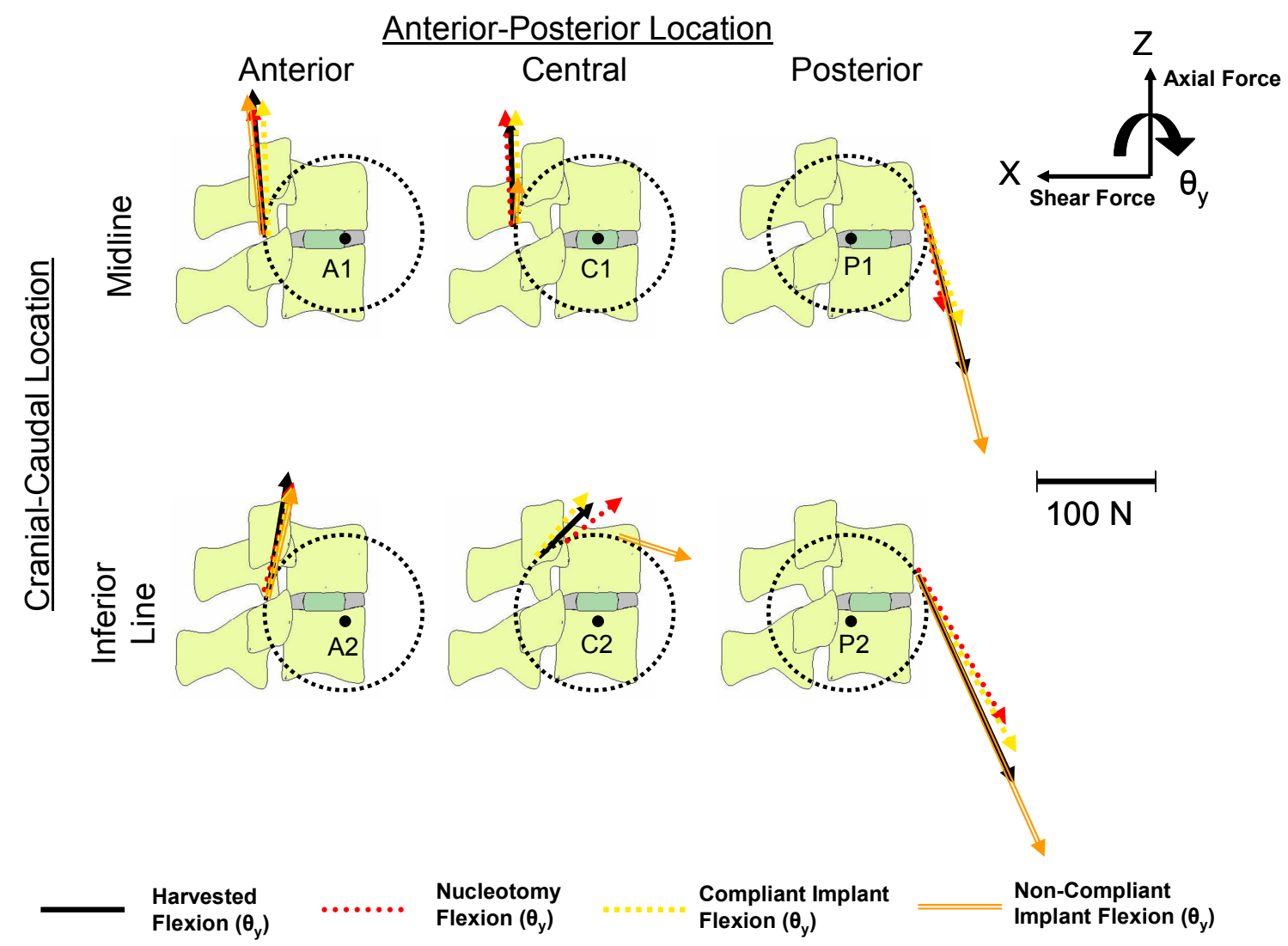

Figure 4.5: Flexion Resultant Force Vectors and Magnitudes, Including Non-

Compliant Implant. The changes in tissue interactions from all three conditions can be garnered from the position and angle of the resultant vector. 


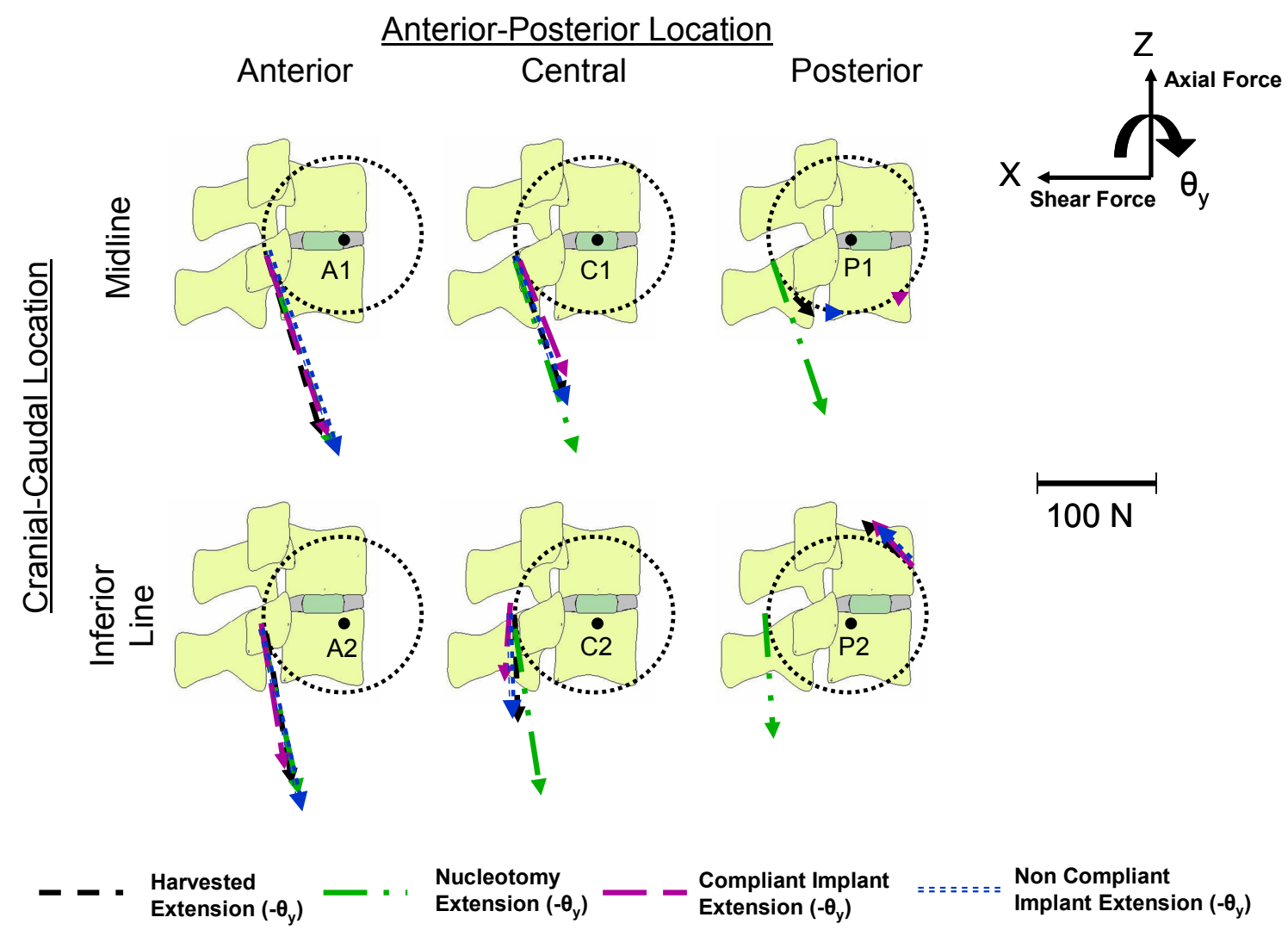

Figure 4.6: Extension Resultant Force Vectors and Magnitudes, Including NonCompliant Implant. The changes in tissue interactions from all three conditions can be garnered from the position and angle of the resultant vector. 
placement of the COR reported in the literature for the lumbar disc is at a central or posterior position. The most significant of the differences found in this study involved the increases in compressive and shear forces at the posterior points of rotation. It has been suggested that mechanical spinal implant devices be designed with a center of rotation that is slightly posterior to match the perceived location of the center of rotation. ${ }^{20 ; 61}$ The increases in compressive and shear forces shown in this study for the non-compliant device must be taken into account in the design of these devices if a posterior center of rotation is chosen. Such information might also be useful towards defining appropriate parameters for bench top mechanical testing protocols and towards establishing design attributes of NA devices.

The role the nucleus plays in resisting compressive forces is well documented. Within a healthy disc, the nucleus acts to distribute and convert external compressive forces to annular tensile forces. The removal of the nucleus, whether through disc degeneration or nucleotomy, changes the ability of the disc to resist and manage compressive forces. ${ }^{23}$ Compliant NA devices are designed to more closely mimic the characteristics of the nucleus. The similarity between the compressive forces of the compliant implant spine condition and harvested spine condition may be attributed to this feature. Non-compliant NA implants, on the other hand, are designed to replicate the motion of the spine, but not necessarily the material and mechanical properties of the disc itself. The ball-and-socket design often used in total disc replacement clearly defines an axis of rotation for the disc. Previous testing of non-compliant total disc replacement technologies demonstrated that non-concentric IAR may lead to an over constrained condition, ${ }^{17}$ resulting in an overloading of adjacent structures and/or inadequate motion restoration. The same results could be expected for non-compliant NA devices.

The effect of shear on the disc and the role the disc plays in resisting shear forces has been studied less. Relative contributions of the disc under posterior shear loading has been reported as $74 \%$ of the ultimate load. ${ }^{62}$ When compared to a denucleated disc, the shear force within the disc was $40 \%$ of the total prior to failure. ${ }^{39}$ The amount of shear force experienced by the disc may be responsible for the expulsion of earlier, compliant NA devices. ${ }^{38}$ Some compliant NA devices may not be constrained within the space left after the nucleotomy. As such, this design may be more susceptible to migration caused by shear forces. Non-compliant implants must be able to resist the increased shear forces resulting from constraints imposed by the device itself, as demonstrated in experiments with total disc replacement devices. ${ }^{32 ; 33}$

While the method used in this study effectively demonstrated that changing the center of rotation had a significant effect on the motion and loading response of the spinal MSU, some limitations do exist and improvements can be made. The use of MSUs to evaluate the devices limits the motions to replicating passive spinal movement due to the lack of muscle involvement. The defined motions are also limited to the sagittal plane. What effects the muscles might have on the NA implants and the motions they cause can only be garnered currently from clinical evaluations. Refining the grid pattern used in this study may also better predict how the devices will behave in vivo. 
Several different types of NA devices are currently being developed. In this study, a spine robot was used to examine two types of NA devices and the effects each had compared to the harvested kinematic response. Using multiple centers of rotation may more effectively evaluate the differences between the types of NA devices, and how each compares to the native tissue response. It can also be used to design better implants by incorporating the knowledge gained from testing different centers of rotation. The data from this study demonstrated that utilizing a robot to change the location of the MSU axis of rotation had a significant effect on the motion and loading responses of the harvested, denucleated, and various implanted lumbar MSUs. 


\section{CHAPTER 5: DISCUSSION}

The alignment grids for all of the specimens are located in Appendix A (Figures A.1-A.7). The tablature data from all four surgical conditions is located in Appendix B (Tables B.1-B.8). Most of the discussion issues for the work done in this thesis have been presented in the previous two chapters in the context of the journal articles. The following discussion concentrates on the total fore vectors shown in Figure 5.1. They represent a combination of the findings presented in Figures 3.6, 3.7, 4.5, and 4.6. As mentioned in Chapters 3 and 4, the force vector direction and placement on the arc of rotation indicates which tissues were responsible for the resultant force. In reality, the resultant force acts from the center of rotation. Tables 5.1 and 5.2 give the numerical values of the force vector magnitudes and angles.

For all four spine conditions, the tissues involved with the resultant forces for the anterior points or rotation (A1 and A2) were similar. Flexion around A1 involved a net tissue tension (positive Fz) and net posterior shear (positive Fx) for all four spine conditions. The resultant force vector indicates that the posterior tissue, specifically the tension of the facet capsule tissue and the posterior ligamentous tissue structures, was most responsible for this result. The fact that the angle of the force vector is almost $90^{\circ}$ indicates that the tensile force is the dominant force. For extension, the posterior structures were again responsible for the resultant force, but in this case, the facets compressed against one another, causing a net compression (negative $\mathrm{Fz}$ ) and anterior shear (negative Fx). Rotation around A2 was different in a couple of aspects. For flexion, the posterior structures were again responsible for the tissue tension. But in this case, they caused an anterior shear. The magnitudes of the forces were also slightly smaller for all the conditions.

Rotation around point $\mathrm{C} 1$ showed few differences between the conditions when comparing the resultant forces. Similar to rotation around the anterior points, the posterior structures were responsible for the resultant force in both flexion and extension testing. In flexion, the magnitude of the resultant force of the non-compliant implant condition was considerably less than the other three conditions. This phenomenon means that even though the tension on the posterior structures was still the dominant force, they were countered by the higher compressive forces caused by the implantation of the noncompliant device. The resultant moment was reached before any more tension could be built. In extension testing, the magnitude of the resultant force of the nucleotomy condition was larger than the other three conditions. This observation matches the results presented in Chapter 3 where the nucleotomy condition had significantly more compression in extension. The same result occurred around for extension rotation about point $\mathrm{C} 2$. Flexion around $\mathrm{C} 2$, however, indicated that something different is occurring in the non-compliant condition. For the other three spine conditions, the tension in the posterior structures was the dominant force. The placement of the resultant force vector on the arc of rotation for the nucleotomy condition, however, indicated that the shear forces towards the anterior structures were more dominate than the posterior tensile structures. It also indicated that the compression of the disc overpowered any tension in 


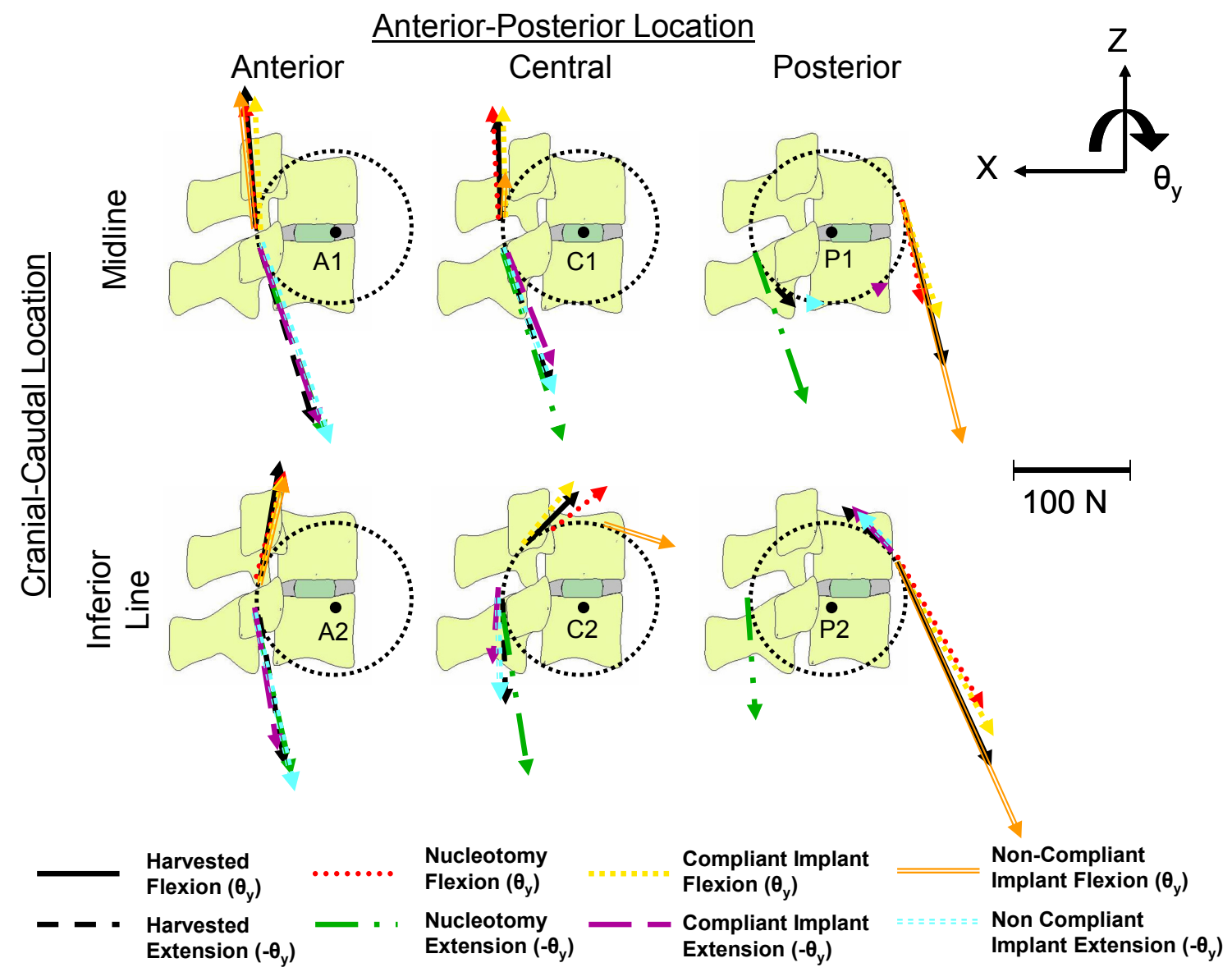

Figure 5.1: All Resultant Force Vectors and Angles. The changes in tissue interactions from all four conditions can be garnered from the position and angle of the resultant vector. 
Table 5.1: Magnitudes and Angles of Resultant Forces for Flexion Testing.

\begin{tabular}{|c|c|c|c|c|c|c|c|c|}
\hline & \multicolumn{4}{|c|}{ Harvested } & \multicolumn{4}{|c|}{ Nucleotomy } \\
\hline Point of Rotation & $\mathrm{Fx}(\mathrm{N})$ & $\mathrm{Fz}(\mathrm{N})$ & Theta & Magnitude & Fx $(\mathrm{N})$ & $\mathrm{Fz}(\mathrm{N})$ & Theta & Magnitude \\
\hline $\mathrm{A} 1$ & 17.82 & 214.70 & 85.26 & 215.44 & 16.52 & 189.87 & 85.03 & 190.58 \\
\hline $\mathrm{A} 2$ & -28.00 & 181.00 & -81.21 & 183.15 & -40.00 & 153.15 & -75.36 & 158.29 \\
\hline $\mathrm{C} 1$ & 7.70 & 158.10 & 87.21 & 158.29 & 8.22 & 165.88 & 87.16 & 166.08 \\
\hline $\mathrm{C} 2$ & -78.60 & 78.50 & -44.96 & 111.09 & -81.10 & 65.50 & -38.93 & 104.24 \\
\hline $\mathrm{P} 1$ & -65.40 & -251.60 & 75.43 & 259.96 & -27.84 & -157.30 & 79.96 & 159.75 \\
\hline $\mathrm{P} 2$ & -141.50 & -313.00 & 65.67 & 343.50 & -130.94 & -235.26 & 60.90 & 269.25 \\
\hline & \multicolumn{4}{|c|}{ Compliant } & \multicolumn{4}{|c|}{ Non-Compliant } \\
\hline Point of Rotation & $\underline{\mathrm{Fx}(\mathrm{N})}$ & $\underline{\mathrm{Fz}(\mathrm{N})}$ & Theta & $\underline{\text { Magnitude }}$ & $\underline{\mathrm{Fx}(\mathrm{N})}$ & $\underline{\mathrm{Fz}(\mathrm{N})}$ & Theta & Magnitude \\
\hline $\mathrm{A} 1$ & 6.94 & 202.20 & 88.03 & 202.32 & 20.25 & 208.40 & 84.45 & 209.38 \\
\hline $\mathrm{A} 2$ & -33.79 & 158.69 & -77.98 & 162.24 & -38.89 & 162.55 & -76.54 & 167.14 \\
\hline $\mathrm{C} 1$ & 2.55 & 164.29 & 89.11 & 164.31 & -3.35 & 68.65 & -87.21 & 68.73 \\
\hline $\mathrm{C} 2$ & -78.37 & 93.77 & -50.11 & 122.21 & -107.35 & -34.83 & 17.98 & 112.86 \\
\hline $\mathrm{P} 1$ & -50.46 & -174.81 & 73.90 & 181.94 & -92.17 & -363.64 & 75.78 & 375.14 \\
\hline $\mathrm{P} 2$ & -142.39 & -257.19 & 61.03 & 293.98 & -164.61 & -425.50 & 68.85 & 456.23 \\
\hline
\end{tabular}


Table 5.2: Magnitudes and Angles of Resultant Forces for Extension Testing.

\begin{tabular}{|c|c|c|c|c|c|c|c|c|}
\hline & \multicolumn{4}{|c|}{ Harvested } & \multicolumn{4}{|c|}{ Nucleotomy } \\
\hline Point of Rotation & $\mathrm{Fx}(\mathrm{N})$ & $\mathrm{Fz}(\mathrm{N})$ & Theta & Magnitude & Fx $(\mathrm{N})$ & $\mathrm{Fz}(\mathrm{N})$ & Theta & Magnitude \\
\hline $\mathrm{A} 1$ & -83.80 & -263.50 & 72.36 & 276.50 & -86.70 & -256.38 & 71.32 & 270.64 \\
\hline $\mathrm{A} 2$ & -37.20 & -220.70 & 80.43 & 223.81 & -52.90 & -243.96 & 77.76 & 249.63 \\
\hline $\mathrm{C} 1$ & -71.00 & -210.30 & 71.34 & 221.96 & -88.36 & -282.08 & 72.61 & 295.59 \\
\hline $\mathrm{C} 2$ & -3.50 & -154.70 & 88.70 & 154.74 & -37.58 & -244.31 & 81.26 & 247.18 \\
\hline $\mathrm{P} 1$ & -29.10 & -30.30 & 46.16 & 42.01 & -77.30 & -229.95 & 71.42 & 242.60 \\
\hline $\mathrm{P} 2$ & 56.30 & 49.80 & 41.49 & 75.16 & -10.77 & -181.66 & 86.61 & 181.98 \\
\hline & \multicolumn{4}{|c|}{ Compliant } & \multicolumn{4}{|c|}{ Non-Compliant } \\
\hline Point of Rotation & $\underline{\mathrm{Fx}(\mathrm{N})}$ & $\underline{\mathrm{Fz}(\mathrm{N})}$ & $\underline{\text { Theta }}$ & Magnitude & $\underline{\mathrm{Fx}(\mathrm{N})}$ & $\underline{\mathrm{Fz}}(\mathrm{N})$ & Theta & Magnitude \\
\hline A1 & -88.31 & -266.33 & 71.65 & 280.59 & -107.31 & -303.72 & 70.54 & 322.12 \\
\hline $\mathrm{A} 2$ & -35.45 & -214.05 & 80.60 & 216.96 & -57.69 & -269.77 & 77.93 & 275.87 \\
\hline $\mathrm{C} 1$ & -69.22 & -169.70 & 67.81 & 183.27 & -78.25 & -219.65 & 70.39 & 233.17 \\
\hline $\mathrm{C} 2$ & 7.08 & -116.81 & -86.53 & 117.03 & -0.65 & -156.23 & 89.76 & 156.23 \\
\hline P1 & -19.77 & 10.07 & -26.99 & 22.18 & -8.91 & -2.58 & 16.17 & 9.27 \\
\hline $\mathrm{P} 2$ & 62.29 & 73.63 & 49.77 & 96.44 & 56.82 & 55.79 & 44.48 & 79.63 \\
\hline
\end{tabular}


the posterior structures. The loss of disc height due to the nucleotomy could account for this change in forces.

Observation of the rotations about the posterior points (P1 and P2) yields interesting information about the tissues involved. The magnitude of the resultant force for the non-compliant implant condition was greater than the other three conditions at both points in flexion. This again indicated that the compressive force was greater for the non-compliant device than for the other conditions as stated in Chapter 4. Extension testing around point $\mathrm{P} 2$, however, indicated a dramatic change in tissue interaction. The harvested condition and both device conditions showed a dominance of tension in the anterior structures. The nucleotomy condition, however, showed a strong compressive force in the posterior structures. In this case, the interaction of the facets and the spinous processes overpowered the tension forces of the disc. As before, the loss of disc height due to the nucleotomy could explain this shift in forces. 


\section{CHAPTER 6: CONCLUSIONS AND RECOMMENDATIONS}

The testing methodology used in this research can effectively characterize the lack of a defined center of rotation in the lumbar disc during flexion and extension. When a nucleotomy was performed, a more destabilized condition of the MSU was noted. It was illustrated by greater rotation in flexion and greater disc compression in extension. The amount of translation along the disc axis also fell within the definition of clinical instability. When a compliant nucleus arthroplasty device was implanted, the response profile returned more towards the harvested condition. The non-compliant implant response profile was also more similar to the harvested condition. But, the noncompliant implant showed an increase in the amount of compressive and shear forces compared to both the harvested condition and the compliant device. These increases in forces must be considered in the design of the implant itself. This testing method offers new insight into understanding the kinematics response of all types of nucleus arthroplasty technologies.

Some limitations do exist in this research. First as mentioned in previous chapters, the lack of muscle involvement in the MSU prevented the true representation of the dynamics of the joint. But, as also mentioned previously, it does represent a passive condition, which can be used to establish the kinematics behavior of the joint. While the points on the grid were designed to mimic the lack of a defined center of rotation in the disc, they cannot completely reproduce the physiologic motion of the spine. If a different grid of points were used for testing, however, similar trends in the differences between the spine conditions would be expected. When placing different NA devices, the placement was only verified visually; no radiographical analysis was performed. Therefore, the orientation of the device itself within the disc space may have influenced the performance. Varying the order of surgical procedures would also help confirm the differences shown in this study between the spine conditions. Also, the average age of the cadaveric tissue was not that of the target population for nucleus arthroplasty technologies. Finally, as mentioned previously, the motion of the MSU was limited to the sagittal plane.

One recommendation for a future study would be to repeat this entire study for the cervical spine. While nucleus replacement technology is currently restricted to the lumbar spine, disc degeneration also occurs in the cervical spine. Investigating the influence of the surgical procedures in the cervical spine would be beneficial. Another recommendation involves total disc replacements. This testing protocol is not limited to testing nucleus replacement devices, but can also be applied to total disc replacement

devices. Some of the total disc replacement technologies incorporate non-compliant and compliant elements in their designs. Using the grid technique when testing these different designs could aid in the matching of the axes of rotation, and help designers manufacture a better implant.

While further studies are needed to completely understand the spinal nucleus and nucleus replacement technology, the method used in this study is one step in the process of designing an evaluating an implant for early disc degeneration treatment. 


\section{LIST OF REFERENCES}

1. Nucleus Arthroplasty Technology in Spinal Care Volume I: Fundamentals. Davis RJ and Girardi FP (eds). Minneapolis, MN: Raymedica, LLC, 2006.

2. Nucleus Arthroplasty Technology in Spinal Care Volume II: Biomechanics and Development. Davis RJ, Girardi FP, Cammisa, FP, and Hutton, WC (eds). Minneapolis, MN: Raymedica, LLC, 2006.

3. Nucleus Arthroplasty Technology in Spinal Care Volume III: Surgical Techniques and Technologies. Davis RJ, Girardi FP, and Cammisa FP (eds). Minneapolis, MN: Raymedica, LLC, 2006.

4. Nucleus Arthroplasty Technology in Spinal Care Volume IV: Emerging Technology. Davis RJ, Girardi FP, and Cammisa FP (eds). Minneapolis, MN: Raymedica, LLC, 2006.

5. Bao QB, McCullen GM, and Higham PA. The artificial disc: theory, design and materials. Biomaterials 1996;17:1157-67.

6. Bao QB and Yaun HA. New technologies in spine: nucleus replacement. Spine 2002;27:1245-7.

7. Battie MC and Videman T. Lumbar disc degeneration: epidemiology and genetics. J Bone Joint Surg Am 2006;88 Suppl 2:3-9.

8. Battie MC, Videman T, and Parent E. Lumbar disc degeneration: epidemiology and genetic influences. Spine 2004;29:2679-90.

9. Bertagnoli R, Karg A, and Voigt S. Lumbar partial disc replacement. Orthop Clin North Am 2005;36:341-7.

10. Bertagnoli R, Sabatino CT, Edwards JT, Gontarz GA, Prewett A, and Parsons JR. Mechanical testing of a novel hydrogel nucleus replacement implant. Spine J 2005;5:672-81.

11. Boden SD, Davis DO, Dina TS, Patronas NJ, and Wiesel SW. Abnormal magnetic-resonance scans of the lumbar spine in asymptomatic subjects. A prospective investigation. J Bone Joint Surg Am 1990;72:403-8.

12. Bonin, $\mathrm{H}$. In vitro anaysis of the instantaneous center of rotation in a human cervical spine model using a spine robot. Master's Thesis. The University of Tennessee Health Science Center, 2006.

13. Bono CM and Garfin SR. History and evolution of disc replacement. Spine J 2004;4:145S-50S. 
14. Coric D and Mummaneni PV. Nucleus replacement technologies. J Neurosurg Spine 2008;8:115-20.

15. DePalma AF and Rothman RH. The Intervertebral Disc. Philadelphia: W.B. Saunders Company, 1970.

16. DiAngelo D, Zufelt N, Sander E, and Kelly B. Development of a kinematics based testing protocol to study lumbar disc dynamics. Proc of the 8th Ann Spine Arthro Soc Mtg, 2008.

17. DiAngelo DJ, Foley KT, Morrow B, Kiehm KJ, and Gilmour L. The effects of over-sizing of a disc prosthesis on spine biomechanics. Proc of the 6th Ann Spine Arthro Soc Mtg, 2006.

18. DiMartino A, Vaccaro AR, Lee JY, Denaro V, and Lim MR. Nucleus pulposus replacement: basic science and indications for clinical use. Spine 2005;30:S16S22.

19. Disch AC, Schmoelz W, Matziolis G, Schneider SV, Knop C, and Putzier M. Higher risk of adjacent segment degeneration after floating fusions: long-term outcome after low lumbar spine fusions. J Spinal Disord Tech. 2008;21:79-85.

20. Dooris AP, Goel VK, Grosland NM, Gilbertson LG, and Wilder DG. Loadsharing between anterior and posterior elements in a lumbar motion segment implanted with an artificial disc. Spine 2001;26:E122-E129.

21. Dunlop RB, Adams MA, and Huton WC. Disc space narrowing and the lumbar facet joints. J Bone Joint Surg 1996;66B:706-10.

22. Eysel P, Rompe JD, Schoenmayr R, and Zoellner J. Biomechanical behaviour of a prosthetic lumbar nucleus. Acta Neurochir 1999;141:1083-7.

23. Frei H, Oxland TR, Rathonyi GC, and Nolte LP. The effect of nucleotomy on lumbar spine mechanics in compression and shear loading. Spine 2001;26:2080-9.

24. Goel VK, Panjabi MM, Patwardhan AG, Dooris AP, and Serhan H. Test protocols for evaluation of spinal implants. J Bone Joint Surg Am 2006;88 Suppl 2:103-9.

25. Goel VK, Wilder DG, Pope MH, and Edwards WT. Biomechanical testing of the spine. Load-controlled versus displacement-controlled analysis. Spine 1995;20:2354-7.

26. Gower WE and Pedrini V. Age-related variations in proteinpolysaccharides from human nucleus pulposus, annulus fibrosus, and costal cartilage. J Bone Joint Surg Am 1969;51A:1154-62.

27. Gray H. Anatomy of the Human Body. 20 ed. Philadelphia: Lee \& Febiger, 1918. 
28. Greenwald RA, Moy WW, and Seibold J. Functional properties of cartilage proteoglycans. Semin Arthritis Rheum 1978;8:53-67.

29. Guyer RD and Ohnmeiss DD. Intervertebral disc protheses. Spine 2003;28:S15S23.

30. Heuer F, Schmidt H, Claes L, and Wilke HJ. Stepwise reduction of functional spinal structures increase vertebral translation and intradiscal pressure. J Biomech 2007;40:795-803.

31. Heuer F, Schmidt H, Klezl Z, Claes L, and Wilke HJ. Stepwise reduction of functional spinal structures increase range of motion and change lordosis angle. $\mathrm{J}$ Biomech 2007;40:271-80.

32. Huang RC, Girardi FP, Cammisa FP, Jr., and Wright TM. The implications of constraint in lumbar total disc replacement. J Spinal Disord Tech 2003;16:412-7.

33. Huang RC, Wright TM, Panjabi MM, and Lipman JD. Biomechanics of nonfusion implants. Orthop Clin North Am 2005;36:271-80.

34. Humzah MD and Soames RW. Human intervertebral disc: structure and function. Anat Rec 1988;220:337-56.

35. Kelly B, DiAngelo D, and Foley K. Design of a multi-axis programmable spine robot for the study of multi-body spinal biomechanics. 32nd Ann Mtg of the Cerv Spine Res Soc, 2004.

36. Kirkaldy-Willis WH and Farfan HF. Instability of the lumbar spine. Clin Orthop Relat Res 1982;110-23.

37. Kirkaldy-Willis WH, Wedge JH, Yong-Hing K, and Reilly J. Pathology and pathogenesis of lumbar spondylosis and stenosis. Spine 1978;3:319-28.

38. Klara PM and Ray CD. Artificial nucleus replacement: clinical experience. Spine 2002;27:1374-7.

39. Lantz SA, Lafferty JF, and Bowman DA. Response of the intervertebral disk of the rhesus monkey to P-A shear stress. J Biomech Eng 1980;102:137-40.

40. Lawrence JS. Disc degeneration. Its frequency and relationship to symptoms. Ann Rheum Dis 1969;28:121-38.

41. Lee CK and Langrana NA. Lumbosacral spinal fusion. A biomechanical study. Spine 1984;9:574-81.

42. Leong JC, Chun SY, Grange WJ, and Fang D. Long-term results of lumbar intervertebral disc prolapse. Spine 1983;8:793-9. 
43. Luoma K, Riihimaki H, Luukkonen R, Raininko R, Viikari-Juntura E, and Lamminen A. Low back pain in relation to lumbar disc degeneration. Spine 2000;25:487-92.

44. Meakin JR. Replacing the nucleus pulposus of the intervertebral disk: prediction of suitable properties of a replacement material using finite element analysis. J Mater Sci Mater Med 2001;12:207-13.

45. Meakin JR, Redpath TW, and Hukins DW. The effect of partial removal of the nucleus pulposus from the intervertebral disc on the response of the human annulus fibrosus to compression. Clin Biomech 2001;16:121-8.

46. Niosi CA and Oxland TR. Degenerative mechanics of the lumbar spine. Spine J 2004;4:202S-8S.

47. Panjabi MM. Biomechanical evaluation of spinal fixation devices: a conceptualframework. Spine 1988;13:1129-34.

48. Panjabi MM. Clinical spinal instability and low back pain. J Electromyogr Kinesiol 2003;13:371-9.

49. Panjabi MM. Hybrid multidirectional test method to evaluate spinal adjacent-level effects. Clin Biomech 2007;22:257-65.

50. Pearcy MJ and Bogduk N. Instantaneous axes of rotation of the lumbar intervertebral joints. Spine 1988;13:1033-41.

51. Pearcy MJ and Tibrewal SB. Axial rotation and lateral bending in the normal lumbar spine measured by three-dimensional radiography. Spine 1984;9:582-7.

52. Pearcy MJ and Tibrewal SB. Lumbar intervertebral disc and ligament deformations measured in vivo. Clin Orthop Relat Res 1984;281-6.

53. Porterfield JA and DeRosa C. Mechanical Neck Pain: Perspectives in Functional Anatomy. 1st ed. Philadelphia: W.B. Saunders Company, 1995.

54. Rengachary SS and Balabhadra RS. Black disc disease: a commentary. Neurosurg Focus 2002;13:E14.

55. Rousseau MA, Bradford DS, Bertagnoli R, Hu SS, and Lotz JC. Disc arthroplasty design influences intervertebral kinematics and facet forces. Spine J 2006;6:25866.

56. Sakalkale DP, Bhagia SA, and Slipman CW. A historical review and current perspective on the intervertebral disc prothesis. Pain Physician 2003;6:195-8.

57. Sakamaki T, Katoh S, and Sairyo K. Normal and spondylolytic pediatric spine movements with reference to instantaneous axis of rotation. Spine 2002;27:141-5. 
58. Schneider G, Pearcy MJ, and Bogduk N. Abnormal motion in spondylolytic spondylolisthesis. Spine 2005;30:1159-64.

59. Szpalski M, Gunzburg R, and Mayer M. Spine arthroplasty: a historical review. Eur Spine J 2002;11 Suppl 2:S65-S84.

60. Vigna FE and McAfee PC. Nonfusion alternatives: artificial disks and prosthetic nuclear replacement in the thoracolumbar spine. Curr Opin Orthop 2004;15:15966.

61. White AA, III and Panjabi MM. Clinical Biomechanics of the Spine. 2nd ed. Philadelphia: J.B. Lippincott Co., 1990.

62. Yingling VR and McGill SM. Mechanical properties and failure mechanics of the spine under posterior shear load: observations from a porcine model. J Spinal Disord 1999;12:501-8. 
APPENDIX A: SPECIMEN ALIGNMENT GRIDS

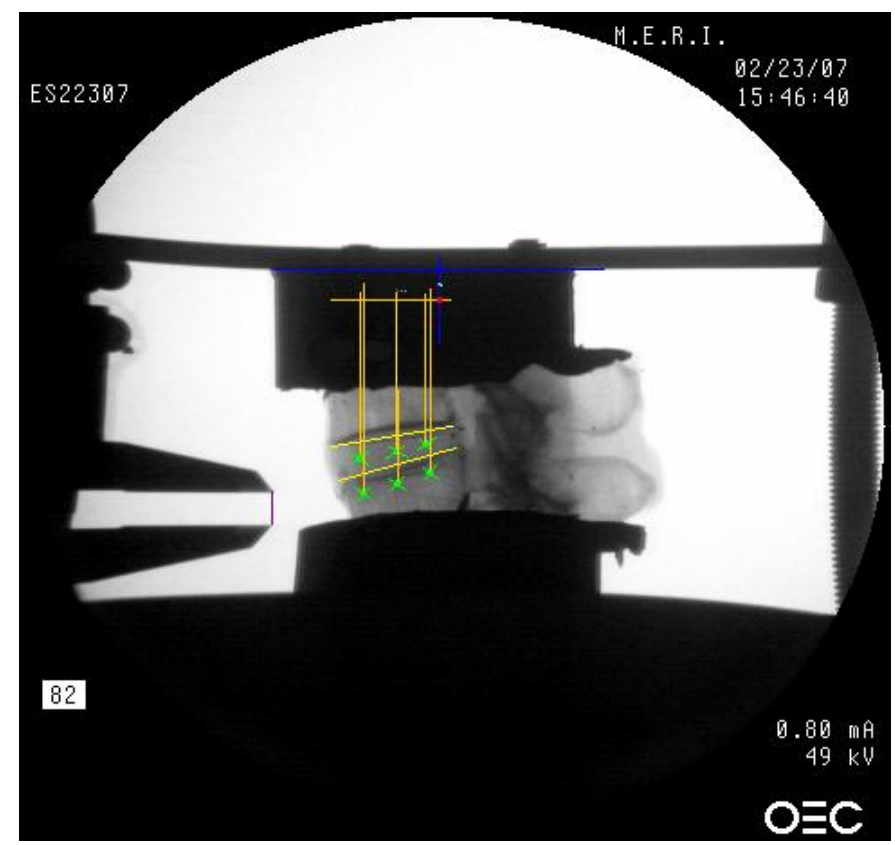

Figure A.1: Grid for Specimen 0105416 L3-L4.

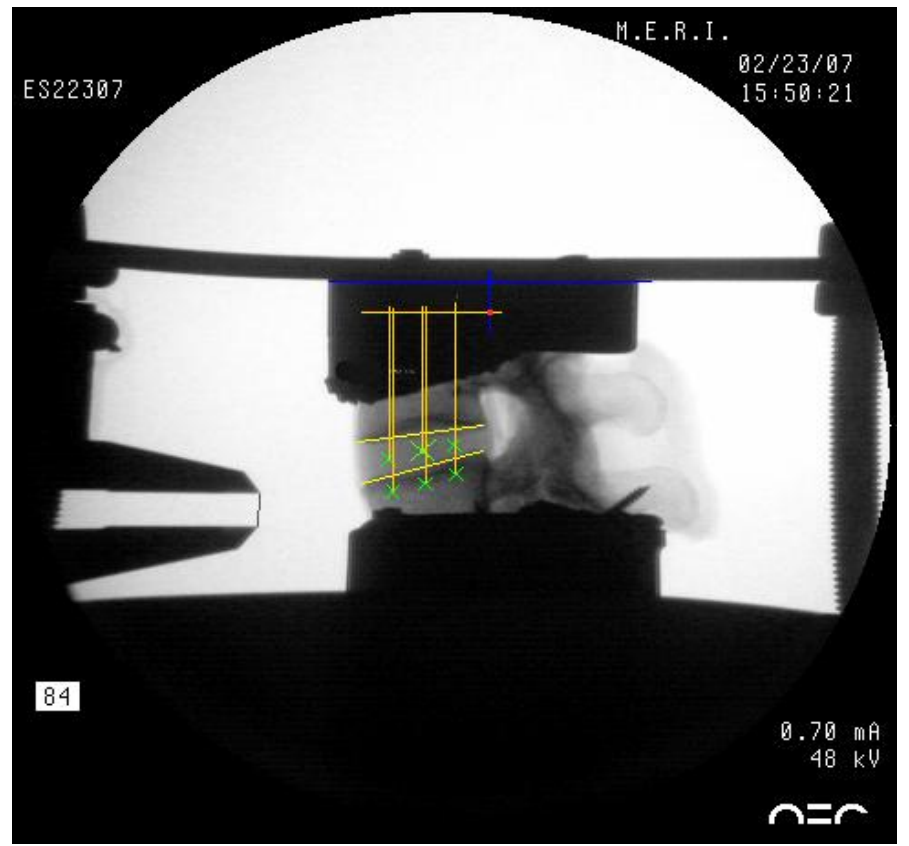

Figure A.2: Grid for Specimen ADS0612051 L3-L4. 


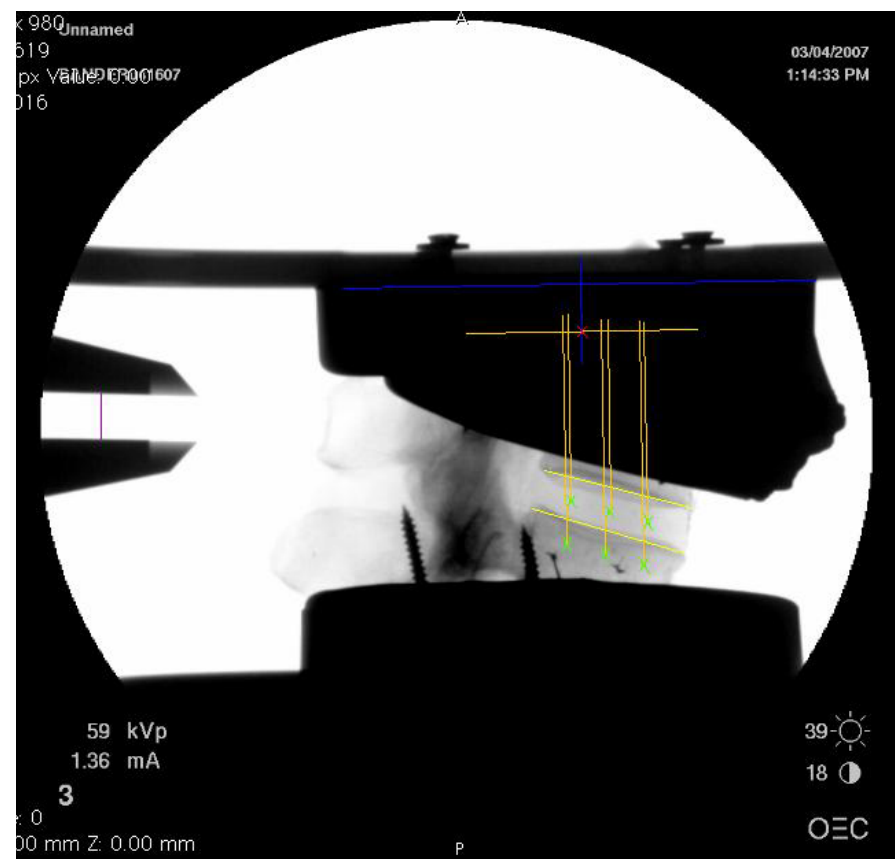

Figure A.3: Grid for Specimen DRT050786 L3-L4.

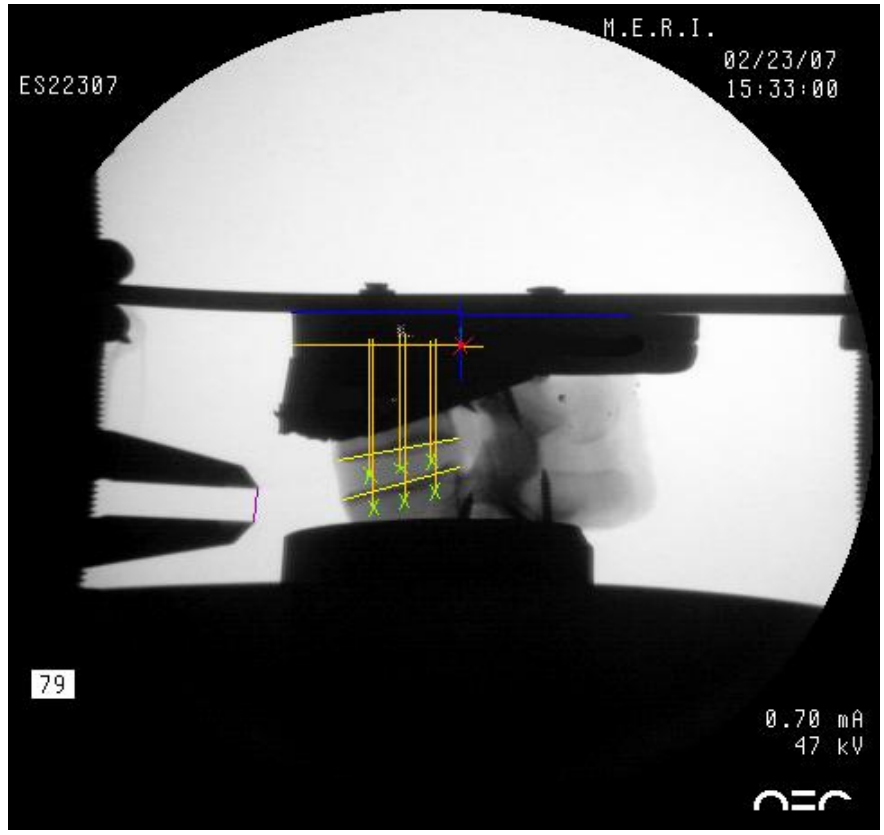

Figure A.4: Grid for Specimen DRT060069 L3-L4. 


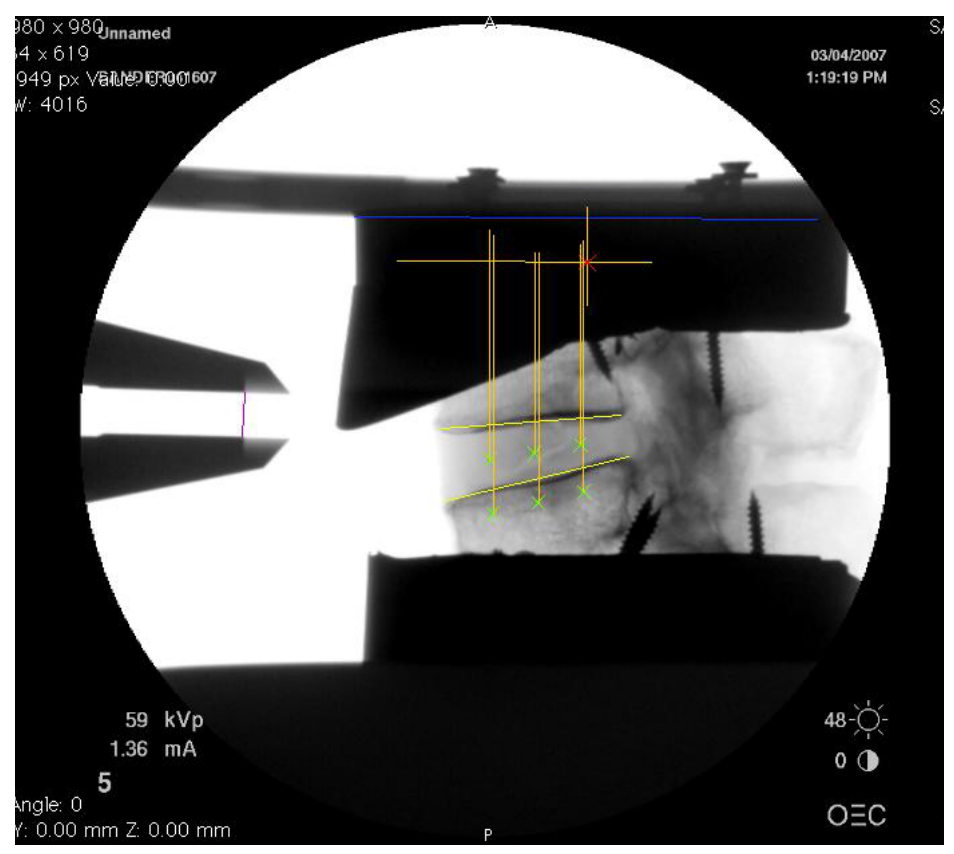

Figure A.5: Grid for Specimen UF01A030 L3-L4.

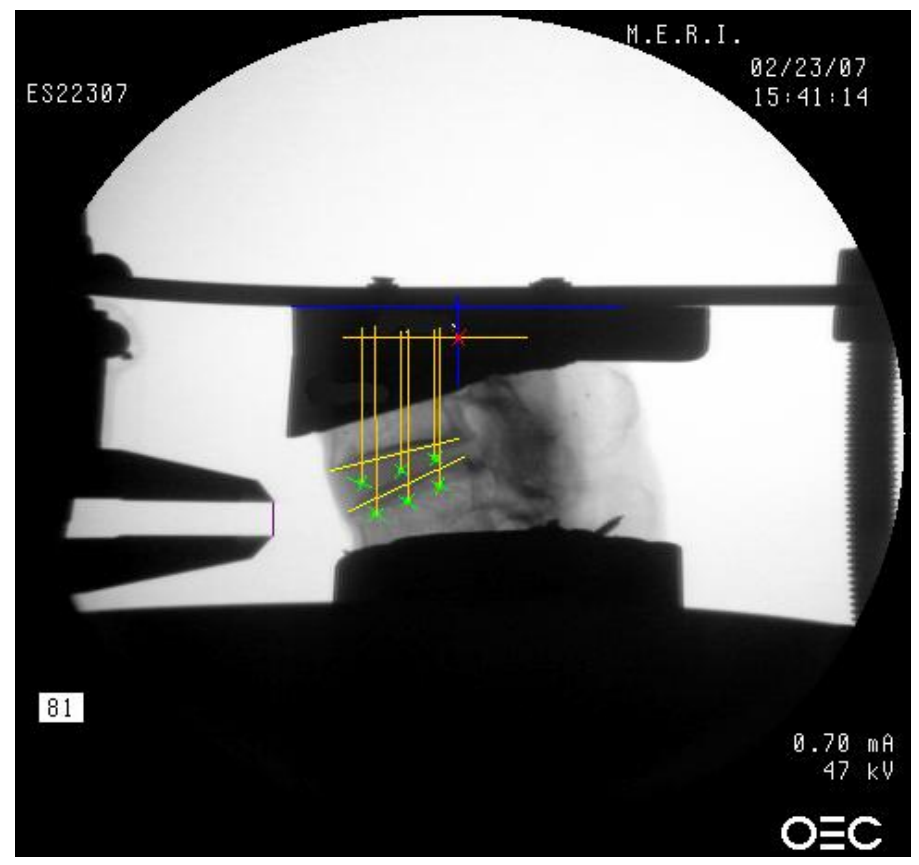

Figure A.6: Grid for Specimen UJ00D35 L3-L4. 


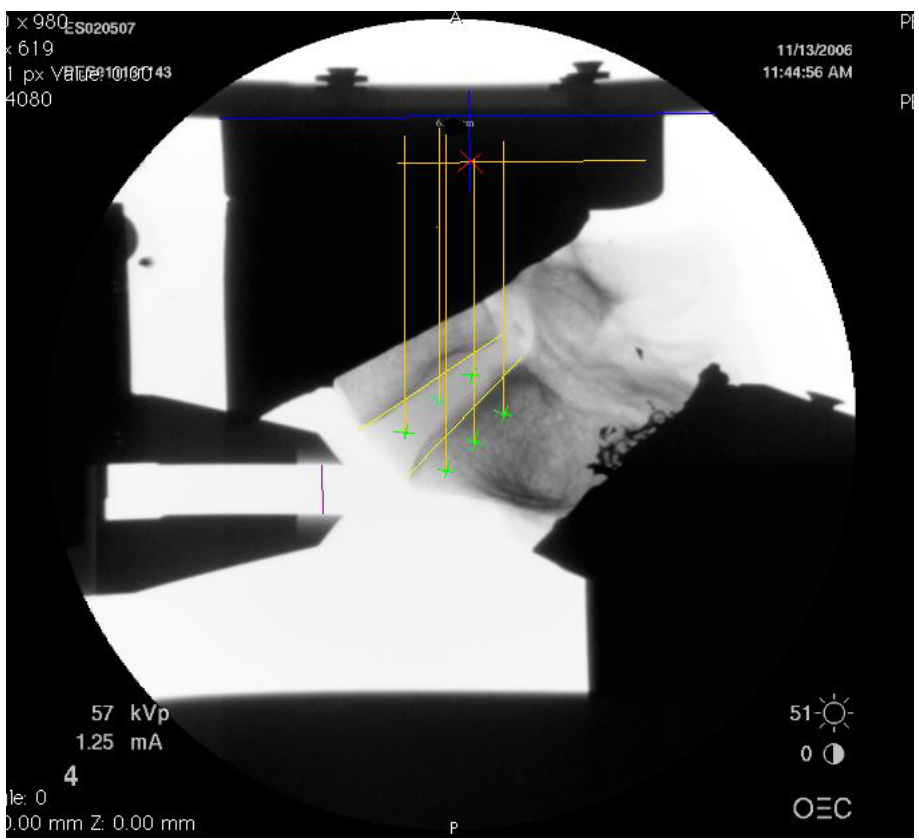

Figure A.7: Grid for Specimen ADS0612051 L5-S1. 
APPENDIX B: TABULATURE DATA

Table B.1: Harvested Spine Condition Flexion Data.

\begin{tabular}{|c|c|c|c|c|c|c|c|c|c|c|c|c|c|c|c|c|c|c|c|c|c|}
\hline \multicolumn{11}{|c|}{ Points along Disc Midline } & \multicolumn{11}{|c|}{ Points in Subjacent Body } \\
\hline \multicolumn{11}{|c|}{$\mathrm{A} 1$} & \multicolumn{11}{|c|}{$\mathrm{A} 2$} \\
\hline Spine & Fx & Fy & $\mathrm{Fz}$ & $\mathrm{Mx}$ & My & $\mathrm{Mz}$ & $\mathrm{x}$ & $\mathrm{z}$ & pitch & roll & Spine & Fx & Fy & $\mathrm{Fz}$ & $\mathrm{Mx}$ & My & $\mathrm{Mz}$ & $\mathrm{x}$ & $\mathrm{z}$ & pitch & roll \\
\hline 0105416 L3 -L4 & 12.6 & 20.6 & 245.5 & -1.9 & -7.5 & 0.9 & 24.4 & -38.2 & 3.3 & 0.0 & 0105416 L3 -L4 & -12.1 & 20.2 & 231.5 & -2.1 & -7.4 & 0.7 & 20.3 & -48.9 & 3.5 & 0.0 \\
\hline UJ00D35 L3-L4 & 44.9 & -21.1 & 204.9 & -0.2 & -7.6 & -0.6 & 28.7 & -63.2 & 4.1 & 0.0 & UJ00D35 L3-L4 & -20.9 & -20.6 & 145.2 & 0.5 & -7.7 & -0.5 & 23.3 & -85.9 & 4.1 & 0.0 \\
\hline ADS0612051 L5-S1 & 18.7 & 1.4 & 123.1 & 0.0 & -7.8 & 0.1 & -5.3 & -43.6 & 4.7 & 0.0 & ADS0612051 L5-S1 & -54.7 & 0.9 & 68.3 & 0.5 & -8.0 & -0.3 & -16.4 & -49.3 & 5.8 & 0.0 \\
\hline ADS0612051 L3-L4 & 22.8 & 3.7 & 202.9 & 1.0 & -7.6 & 0.1 & -24.7 & -68.5 & 3.9 & 0.0 & ADS0612051 L3-L4 & -10.2 & -6.2 & 185.5 & 1.1 & -7.5 & -0.1 & -25.7 & -78.4 & 3.8 & 0.0 \\
\hline DRT060069 L3-L4 & 44.4 & 1.7 & 217.1 & 0.1 & -7.7 & 0.0 & -25.2 & -73.6 & 3.5 & 0.0 & DRT060069 L3-L4 & -15.4 & -4.0 & 207.4 & 0.3 & -7.7 & -0.1 & -26.0 & -83.1 & 3.6 & 0.0 \\
\hline DRT050786 L3-L4 & -3.1 & -2.9 & 249.4 & 0.9 & -7.7 & 0.0 & 13.4 & -41.0 & 4.0 & 0.0 & DRT050786 L3-L4 & -41.4 & -5.0 & 204.1 & 0.8 & -7.8 & 0.1 & 12.9 & -50.0 & 3.6 & 0.0 \\
\hline UF01A030 L3-L4 & -15.6 & -44.3 & 259.8 & -0.2 & -7.6 & -0.9 & -34.8 & -76.9 & 3.8 & 0.0 & UF01A030 L3-L4 & -41.4 & -42.9 & 224.9 & 0.5 & -7.7 & -0.9 & -35.6 & -88.4 & 3.4 & 0.0 \\
\hline Mean & 17.8 & -5.8 & 214.7 & 0.0 & -7.6 & -0.1 & -3.4 & -57.9 & 3.9 & 0.0 & Mean & -28.0 & -8.2 & 181.0 & 0.2 & -7.7 & -0.2 & -6.7 & -69.2 & 4.0 & $\overline{0.0}$ \\
\hline Standard Deviation & 22.5 & 20.9 & 46.2 & 1.0 & 0.1 & 0.6 & 25.8 & 16.4 & 0.4 & 0.0 & Standard Deviation & 17.6 & 19.5 & 57.3 & 1.1 & 0.2 & 0.5 & 24.8 & 18.7 & 0.8 & 0.0 \\
\hline \multicolumn{11}{|c|}{$\mathrm{Cl}$} & \multicolumn{11}{|c|}{$\mathrm{C} 2$} \\
\hline Spine & Fx & Fy & $\mathrm{Fz}$ & $\mathrm{Mx}$ & My & $\mathrm{Mz}$ & $\mathrm{x}$ & $\mathrm{z}$ & pitch & roll & Spine & Fx & Fy & $\mathrm{Fz}$ & $\mathrm{Mx}$ & My & $\mathrm{Mz}$ & $\mathrm{x}$ & $\mathrm{z}$ & pitch & roll \\
\hline 0105416 L3 -L4 & & & & & & & & & & & 0105416 L3 -L4 & -40.6 & 21.3 & 199.9 & -2.0 & -7.6 & 0.9 & 11.2 & -46.4 & 4.9 & 0.0 \\
\hline UJ00D35 L3-L4 & 9.0 & -35.5 & 143.5 & 0.4 & -7.9 & -0.6 & 18.1 & -68.0 & 5.5 & 0.0 & UJ00D35 L3-L4 & -91.5 & -28.8 & 1.8 & 1.6 & -7.8 & 0.2 & 13.4 & -82.2 & 5.3 & 0.0 \\
\hline ADS0612051 L5-S1 & 26.8 & 1.4 & 159.4 & -0.1 & -8.0 & 0.3 & 6.4 & -48.5 & 6.9 & 0.0 & ADS0612051 L5-S1 & -100.2 & 2.4 & -19.0 & 1.0 & -8.0 & -0.8 & -20.0 & -44.2 & 6.9 & 0.0 \\
\hline ADS0612051 L3-L4 & 25.2 & -33.6 & 156.9 & 1.0 & -7.7 & -0.7 & -35.2 & -65.4 & 5.4 & 0.0 & ADS0612051 L3-L4 & -58.7 & -31.0 & 86.5 & 1.7 & -7.7 & -0.5 & -36.1 & -76.0 & 5.5 & 0.0 \\
\hline DRT060069 L3-L4 & 62.2 & -0.3 & 214.3 & 0.1 & -7.8 & 0.0 & -34.1 & -72.0 & 5.1 & 0.0 & DRT060069 L3-L4 & -48.3 & -6.2 & 139.5 & 0.3 & -7.9 & -0.1 & -35.7 & -81.6 & 5.5 & 0.0 \\
\hline DRT050786 L3-L4 & -37.3 & -2.7 & 68.8 & 0.7 & -8.0 & -0.2 & 5.9 & -38.3 & 5.1 & 0.0 & DRT050786 L3-L4 & -110.9 & -2.1 & 10.5 & 0.7 & -8.0 & 0.1 & 4.6 & -47.5 & 4.6 & 0.0 \\
\hline UF01A030 L3-L4 & -39.9 & -47.1 & 205.7 & 0.8 & -7.9 & -0.5 & -45.1 & -74.7 & 6.1 & 0.0 & UF01A030 L3-L4 & -100.2 & -45.5 & 130.2 & 1.5 & -7.8 & -0.4 & -45.7 & -85.6 & 5.5 & 0.0 \\
\hline Mean & 7.7 & -19.6 & 158.1 & 0.5 & -7.9 & -0.3 & -14.0 & -61.2 & 5.7 & 0.0 & Mean & -78.6 & -12.9 & 78.5 & 0.7 & -7.8 & -0.1 & -15.5 & -66.2 & 5.5 & 0.0 \\
\hline Standard Deviation & 39.8 & 21.5 & 52.2 & 0.4 & 0.1 & 0.4 & 27.1 & 14.5 & 0.7 & 0.0 & Standard Deviation & 28.6 & 23.1 & 82.9 & 1.3 & 0.1 & 0.6 & 24.9 & 19.1 & 0.7 & 0.0 \\
\hline \multicolumn{11}{|c|}{ P1 } & \multicolumn{11}{|c|}{$\mathrm{P} 2$} \\
\hline Spine & Fx & Fy & $\mathrm{Fz}$ & $\mathrm{Mx}$ & My & $\mathrm{Mz}$ & $\mathrm{x}$ & $\mathrm{z}$ & pitch & roll & Spine & Fx & Fy & $\mathrm{Fz}$ & $\mathrm{Mx}$ & My & $\mathrm{Mz}$ & $\mathrm{x}$ & $\mathrm{z}$ & pitch & roll \\
\hline 0105416 L3 -L4 & -22.4 & 20.4 & -148.7 & -0.9 & -7.8 & 1.6 & 6.0 & -33.2 & 6.2 & 0.0 & 0105416 L3 -L4 & -111.6 & 22.3 & -241.4 & -0.8 & -7.8 & 1.6 & 3.8 & -43.8 & 5.4 & 0.0 \\
\hline UJ00D35 L3-L4 & -57.0 & -44.2 & -214.2 & 1.4 & -7.8 & 0.7 & 8.8 & -64.0 & 5.9 & 0.0 & UJ00D35 L3-L4 & -127.2 & -39.8 & -308.2 & 1.2 & -7.7 & 1.7 & 3.5 & -75.8 & 4.7 & 0.0 \\
\hline ADS0612051 L5-S1 & -93.2 & -5.2 & -33.9 & 0.6 & -8.0 & -0.2 & -18.9 & -32.8 & 8.8 & 0.0 & ADS0612051 L5-S1 & -201.6 & -2.0 & -276.5 & 1.9 & -7.5 & -2.1 & -27.6 & -39.5 & 6.2 & 0.0 \\
\hline ADS0612051 L3-L4 & -26.8 & -102.0 & -140.0 & 0.6 & -7.9 & -0.3 & -45.4 & -63.1 & 5.8 & 0.0 & ADS0612051 L3-L4 & -85.5 & -91.5 & -176.6 & 1.6 & -7.8 & -0.3 & -45.1 & -72.5 & 5.5 & 0.0 \\
\hline DRT060069 L3-L4 & -53.4 & 1.0 & -453.5 & 0.0 & -7.9 & 0.0 & -43.0 & -70.9 & 4.6 & 0.0 & DRT060069 L3-L4 & -145.9 & -1.2 & -513.9 & 0.0 & -7.7 & 0.0 & -44.5 & -80.6 & 3.5 & 0.0 \\
\hline DRT050786 L3-L4 & -118.8 & 12.8 & -403.6 & 0.0 & -8.1 & -0.4 & -2.4 & -35.7 & 4.4 & 0.0 & DRT050786 L3-L4 & -171.7 & 8.6 & -361.7 & 0.1 & -8.1 & -0.2 & -3.4 & -44.7 & 3.9 & 0.0 \\
\hline UF01A030 L3-L4 & -86.5 & -63.0 & -367.1 & 1.8 & -7.8 & 0.3 & -54.7 & -72.9 & 5.9 & 0.0 & UF01A030 L3-L4 & -146.9 & -63.5 & -312.9 & 2.6 & -7.6 & 0.0 & -55.2 & -83.4 & 5.2 & 0.0 \\
\hline Mean & -65.4 & -25.7 & -251.6 & 0.5 & -7.9 & 0.2 & -21.4 & -53.2 & 6.0 & 0.0 & Mean & -141.5 & -23.9 & -313.0 & 0.9 & -7.8 & 0.1 & -24.1 & -62.9 & 4.9 & $\overline{0.0}$ \\
\hline Standard Deviation & 35.6 & 45.3 & 157.6 & 0.9 & 0.1 & 0.7 & 26.4 & 18.4 & 1.5 & 0.0 & Standard Deviation & 38.3 & 42.0 & 106.3 & 1.2 & 0.2 & 1.3 & 25.2 & 19.3 & 0.9 & 0.0 \\
\hline
\end{tabular}


Table B.2: Harvested Spine Condition Extension Data.

\begin{tabular}{|c|c|c|c|c|c|c|c|c|c|c|c|c|c|c|c|c|c|c|c|c|c|}
\hline \multirow{2}{*}{\multicolumn{11}{|c|}{ Points along Disc Midline }} & \multirow{2}{*}{\multicolumn{11}{|c|}{ Points in Subjacent Body }} \\
\hline \multicolumn{7}{|c|}{$\mathrm{A} 1$} & & & & & \multicolumn{5}{|c|}{$\mathrm{A} 2$} & & & & & & \\
\hline Spine & Fx & Fy & $\mathrm{Fz}$ & $\mathrm{Mx}$ & My & $\mathrm{Mz}$ & $\bar{x}$ & $\mathrm{z}$ & pitch & roll & Spine & Fx & Fy & $\mathrm{Fz}$ & Mx & My & $\mathrm{Mz}$ & $\mathrm{x}$ & $\mathrm{z}$ & pitch & roll \\
\hline 0105416 L3 -L4 & -56.7 & 5.8 & -270.5 & 0.3 & 7.8 & 1.7 & 24.6 & -38.0 & -1.9 & 180.0 & 0105416 L3 -L4 & -26.5 & 6.3 & -243.5 & 0.2 & 7.9 & 1.5 & 20.3 & -48.9 & -2.1 & 180.0 \\
\hline UJ00D35 L3-L4 & -118.9 & -28.9 & -276.6 & -2.6 & 7.7 & -0.6 & 29.0 & -63.3 & -1.8 & 180.0 & UJ00D35 L3-L4 & -74.2 & -22.8 & -280.7 & -2.8 & 7.6 & -0.6 & 23.4 & -86.0 & -2.4 & 180.0 \\
\hline ADS0612051 L5-S1 & -170.5 & -14.7 & -232.9 & 0.3 & 8.0 & -0.1 & -4.9 & -43.6 & -2.5 & 180.0 & ADS0612051 L5-S1 & -103.7 & 1.7 & -148.2 & 0.1 & 8.0 & 0.9 & -16.5 & -49.4 & -4.5 & 180.0 \\
\hline ADS0612051 L3-L4 & -16.9 & -89.9 & -183.3 & 0.9 & 7.9 & -1.5 & -24.4 & -68.6 & -2.5 & 180.0 & ADS0612051 L3-L4 & -10.7 & -86.5 & -166.3 & 1.8 & 7.7 & -1.4 & -25.8 & -78.5 & -2.6 & 180.0 \\
\hline DRT060069 L3-L4 & -117.7 & -2.8 & -371.8 & -0.5 & 8.1 & 0.0 & -24.9 & -73.5 & -1.5 & 180.0 & DRT060069 L3-L4 & -61.9 & -2.0 & -339.2 & -0.6 & 8.1 & 0.0 & -26.1 & -83.1 & -1.7 & 180.0 \\
\hline DRT050786 L3-L4 & -51.7 & 2.7 & -255.7 & -0.3 & 7.5 & -2.9 & 13.7 & -40.9 & -2.9 & 180.0 & DRT050786 L3-L4 & 9.5 & 4.1 & -170.0 & -0.4 & 7.6 & -2.7 & 12.8 & -49.8 & -2.8 & 180.0 \\
\hline UF01A030 L3-L4 & -54.1 & -59.1 & -253.6 & 1.6 & 7.8 & -1.2 & -34.5 & -76.9 & -2.1 & 180.0 & UF01A030 L3-L4 & 6.8 & -49.2 & -197.1 & 2.0 & 7.7 & -0.7 & -35.7 & -88.4 & -2.0 & 180.0 \\
\hline Mean & -83.8 & -26.7 & -263.5 & $\overline{0.0}$ & 7.8 & -0.7 & -3.0 & -57.8 & -2.2 & 180.0 & Mean & -37.2 & -21.2 & -220.7 & 0.0 & 7.8 & -0.4 & -6.8 & -69.2 & -2.6 & 180.0 \\
\hline Standard Deviation & 53.3 & 35.8 & 57.0 & 1.3 & 0.2 & 1.4 & 25.8 & 16.5 & 0.5 & 0.0 & Standard Deviation & 43.5 & 35.0 & 70.1 & 1.6 & 0.2 & 1.4 & 24.8 & 18.7 & 0.9 & 0.0 \\
\hline \multicolumn{11}{|c|}{$\mathrm{C} 1$} & \multicolumn{11}{|c|}{$\mathrm{C} 2$} \\
\hline Spine & Fx & Fy & Fz & $\mathrm{Mx}$ & $\mathrm{My}$ & $\mathrm{Mz}$ & $\mathrm{x}$ & $\mathrm{z}$ & pitch & roll & Spine & Fx & Fy & $\mathrm{Fz}$ & $\mathrm{Mx}$ & My & $\mathrm{Mz}$ & $\mathrm{x}$ & $\mathrm{z}$ & pitch & roll \\
\hline 0105416 L3 -L4 & -32.8 & 10.3 & -205.0 & 0.4 & 7.8 & 1.6 & 13.6 & -37.5 & -3.3 & 180.0 & 0105416 L3 -L4 & 26.8 & 9.9 & -178.0 & 0.1 & 7.9 & 1.5 & 11.0 & -46.4 & -3.4 & 180.0 \\
\hline UJ00D35 L3-L4 & -122.5 & -20.7 & -316.1 & -3.2 & 7.3 & -0.4 & 18.6 & -68.0 & -3.3 & 180.0 & UJ00D35 L3-L4 & -53.0 & -10.3 & -235.2 & -2.8 & 7.5 & -0.2 & 13.3 & -82.5 & -3.7 & 180.0 \\
\hline ADS0612051 L5-S1 & -201.0 & -21.3 & -246.9 & -0.4 & 8.0 & 0.6 & -11.9 & -38.7 & -3.8 & 180.0 & ADS0612051 L5-S1 & -80.1 & 5.8 & -103.5 & -0.2 & 8.0 & 0.9 & -19.9 & -44.4 & -5.3 & 180.0 \\
\hline ADS0612051 L3-L4 & 1.5 & -66.0 & -121.7 & 0.8 & 8.0 & -0.3 & -34.7 & -65.4 & -3.3 & 180.0 & ADS0612051 L3-L4 & 19.1 & -59.1 & -109.3 & 1.4 & 7.9 & -0.2 & -36.1 & -76.1 & -3.2 & 180.0 \\
\hline DRT060069 L3-L4 & -109.7 & -1.9 & -398.8 & -0.9 & 7.7 & 0.0 & -33.7 & -71.9 & -3.0 & 180.0 & DRT060069 L3-L4 & -21.9 & -2.3 & -271.7 & -1.0 & 8.0 & 0.0 & -35.6 & -81.5 & -3.1 & 180.0 \\
\hline DRT050786 L3-L4 & -3.2 & -5.4 & -79.9 & -0.4 & 7.4 & -3.0 & 6.2 & -38.2 & -3.6 & 180.0 & DRT050786 L3-L4 & 54.9 & 5.6 & -63.9 & -0.3 & 7.6 & -2.6 & 4.6 & -47.4 & -3.5 & 180.0 \\
\hline UF01A030 L3-L4 & -29.4 & -61.1 & -103.4 & 1.3 & 7.9 & -0.5 & -44.6 & -74.8 & -2.9 & 180.0 & UF01A030 L3-L4 & 29.7 & -49.2 & -121.1 & 2.0 & 7.8 & 0.1 & -45.6 & -85.6 & -2.8 & 180.0 \\
\hline Mean & -71.0 & -23.7 & -210.3 & -0.3 & 7.8 & $\begin{array}{c}-0.3 \\
\end{array}$ & -12.3 & -56.4 & -3.3 & 180.0 & Mean & -3.5 & -14.2 & -154.7 & -0.1 & 7.8 & -0.1 & -15.5 & -66.3 & -3.6 & 180.0 \\
\hline Standard Deviation & 75.4 & 29.4 & 118.6 & 1.4 & 0.3 & 1.4 & 25.7 & 17.3 & 0.3 & 0.0 & Standard Deviation & 49.3 & 28.2 & 76.1 & 1.6 & 0.2 & 1.3 & 24.8 & 19.1 & 0.8 & 0.0 \\
\hline \multicolumn{11}{|c|}{$\mathrm{P} 1$} & \multicolumn{11}{|c|}{$\mathrm{P} 2$} \\
\hline Spine & Fx & Fy & Fz & $\mathrm{Mx}$ & $\mathrm{My}$ & $\mathrm{Mz}$ & $\mathrm{x}$ & $\mathrm{z}$ & pitch & roll & Spine & Fx & Fy & Fz & $\mathrm{Mx}$ & My & $\mathrm{Mz}$ & $\mathrm{x}$ & $\mathrm{z}$ & pitch & roll \\
\hline 0105416 L3 -L4 & -15.5 & 12.8 & -32.1 & -0.1 & 7.9 & 1.4 & 6.3 & -33.1 & -4.0 & 180.0 & 0105416 L3 -L4 & 54.0 & 15.2 & 10.0 & -0.5 & 7.9 & 1.2 & 3.4 & -44.0 & -3.9 & 180.0 \\
\hline UJ00D35 L3-L4 & -105.0 & -12.4 & -224.6 & -2.5 & 7.6 & -0.2 & 9.0 & -64.3 & -4.8 & 180.0 & UJ00D35 L3-L4 & 30.4 & -4.2 & -28.1 & -1.0 & 8.0 & -0.1 & 3.2 & -76.1 & -5.4 & 180.0 \\
\hline ADS0612051 L5-S1 & -160.5 & -18.7 & -173.8 & -0.8 & 7.9 & 1.3 & -18.4 & -33.0 & -5.2 & 180.0 & ADS0612051 L5-S1 & -5.9 & 9.4 & -5.3 & -0.3 & 7.9 & 0.8 & -28.0 & -39.7 & -6.2 & 180.0 \\
\hline ADS0612051 L3-L4 & 37.5 & -35.0 & 62.1 & 0.7 & 8.0 & 0.8 & -45.2 & -63.3 & -3.4 & 180.0 & ADS0612051 L3-L4 & 57.4 & -28.1 & 109.4 & 0.9 & 8.0 & 0.6 & -45.5 & -72.6 & -3.2 & 180.0 \\
\hline DRT060069 L3-L4 & -31.7 & -3.7 & -83.9 & -1.2 & 8.0 & 0.0 & -42.7 & -70.9 & -4.1 & 180.0 & DRT060069 L3-L4 & 75.8 & 0.7 & 19.0 & -1.2 & 7.9 & -0.1 & -44.5 & -80.6 & -3.9 & 180.0 \\
\hline DRT050786 L3-L4 & 58.0 & -7.9 & 137.1 & -0.3 & 7.7 & -2.1 & -2.4 & -35.8 & -3.8 & 180.0 & DRT050786 L3-L4 & 103.1 & -0.4 & 135.8 & -0.2 & 7.7 & -2.2 & -3.8 & -44.8 & -3.5 & 180.0 \\
\hline UF01A030 L3-L4 & 13.4 & -58.7 & 103.0 & 1.0 & 7.9 & 0.4 & -54.6 & -73.1 & -3.0 & 180.0 & UF01A030 L3-L4 & 79.5 & -50.3 & 108.0 & 1.5 & 7.9 & 0.8 & -55.5 & -83.7 & -2.9 & 180.0 \\
\hline Mean & -29.1 & -17.6 & -30.3 & -0.4 & 7.9 & 0.2 & -21.1 & -53.4 & -4.0 & 180.0 & Mean & 56.3 & -8.3 & 49.8 & -0.1 & 7.9 & 0.1 & -24.4 & -63.1 & -4.1 & 180.0 \\
\hline Standard Deviation & 78.6 & 23.2 & 138.8 & 1.2 & 0.1 & 1.2 & 26.4 & 18.5 & 0.8 & 0.0 & Standard Deviation & 35.7 & 23.0 & 65.8 & 1.0 & 0.1 & 1.1 & 25.2 & 19.3 & 1.2 & 0.0 \\
\hline
\end{tabular}


Table B.3: Nucleotomy Spine Condition Flexion Data.

\begin{tabular}{|c|c|c|c|c|c|c|c|c|c|c|c|c|c|c|c|c|c|c|c|c|c|}
\hline \multicolumn{11}{|c|}{ Points along Disc Midline } & \multicolumn{11}{|c|}{ Points in Subjacent Body } \\
\hline \multicolumn{11}{|c|}{$\mathrm{A} 1$} & \multicolumn{11}{|c|}{$\mathrm{A} 2$} \\
\hline Spine & $\mathrm{Fx}$ & Fy & $\mathrm{Fz}$ & $\mathrm{Mx}$ & $\mathrm{My}$ & $\mathrm{Mz}$ & $\bar{x}$ & $\mathrm{z}$ & pitch & roll & Spine & Fx & Fy & $\mathrm{Fz}$ & Mx & My & $\mathrm{Mz}$ & $\mathrm{x}$ & $\mathrm{z}$ & pitch & roll \\
\hline 0105416 L3 -L4 & -26.1 & 23.3 & 216.6 & -1.8 & -7.7 & 0.5 & 24.1 & -39.0 & 4.9 & 0.0 & 0105416 L3 -L4 & -46.5 & 24.1 & 192.5 & -1.9 & -7.7 & 0.6 & 20.0 & -49.9 & 4.9 & 0.0 \\
\hline UJ00D35 L3-L4 & 71.7 & -26.1 & 182.2 & -0.4 & -7.6 & -0.8 & 28.8 & -63.6 & 5.0 & 0.0 & UJ00D35 L3-L4 & -36.8 & -25.1 & 123.8 & 0.8 & -7.7 & -0.4 & 23.4 & -86.5 & 5.3 & 0.0 \\
\hline ADS0612051 L5-S1 & 2.0 & 1.7 & 118.5 & 0.1 & -7.9 & 0.0 & 13.6 & -54.5 & 7.5 & 0.0 & ADS0612051 L5-S1 & -59.6 & 3.0 & 62.1 & 0.6 & -7.9 & -1.0 & 2.3 & -60.3 & 8.8 & 0.0 \\
\hline ADS0612051 L3-L4 & 9.4 & -73.5 & 153.2 & 0.3 & -6.8 & -3.8 & -28.2 & -71.6 & 6.4 & 0.0 & ADS0612051 L3-L4 & -27.5 & -81.1 & 131.8 & 1.2 & -6.4 & -4.3 & -29.1 & -81.5 & 6.3 & 0.0 \\
\hline DRT060069 L3-L4 & 55.8 & 4.2 & 211.0 & 0.3 & -7.6 & 0.1 & -25.3 & -74.8 & 5.7 & 0.0 & DRT060069 L3-L4 & -28.8 & -1.4 & 196.1 & 0.4 & -7.7 & 0.0 & -26.2 & -84.4 & 6.0 & 0.0 \\
\hline DRT050786 L3-L4 & -8.2 & -33.0 & 218.2 & 0.8 & -7.8 & -0.4 & 19.5 & -42.3 & 7.8 & 0.0 & DRT050786 L3-L4 & -29.0 & -23.4 & 180.6 & 1.1 & -7.8 & -0.2 & 18.9 & -51.9 & 8.4 & 0.0 \\
\hline UF01A030 L3-L4 & 10.9 & -20.9 & 229.3 & 1.5 & -7.6 & -0.4 & -35.9 & -77.3 & 4.9 & 0.0 & UF01A030 L3-L4 & -51.8 & -20.8 & 185.2 & 1.8 & -7.5 & -0.3 & -36.7 & -89.0 & 4.8 & 0.0 \\
\hline Mean & 16.5 & -17.8 & 189.9 & 0.1 & -7.6 & -0.7 & -0.5 & -60.4 & 6.1 & 0.0 & Mean & -40.0 & -17.8 & 153.2 & 0.6 & -7.5 & -0.8 & -3.9 & -71.9 & 6.3 & $\overline{0.0}$ \\
\hline Standard Deviation & 34.9 & 31.5 & 40.9 & 1.0 & 0.4 & 1.4 & 28.0 & 15.5 & 1.2 & 0.0 & Standard Deviation & 12.7 & 33.1 & 49.6 & 1.2 & 0.5 & 1.6 & 26.1 & 17.2 & 1.6 & 0.0 \\
\hline \multicolumn{11}{|c|}{$\mathrm{C} 1$} & \multicolumn{11}{|c|}{$\mathrm{C} 2$} \\
\hline Spine & Fx & Fy & $\mathrm{Fz}$ & $\mathrm{Mx}$ & $\mathrm{My}$ & $\mathrm{Mz}$ & $\mathrm{x}$ & $\mathrm{z}$ & pitch & roll & Spine & Fx & Fy & Fz & $\mathrm{Mx}$ & My & $\mathrm{Mz}$ & $\mathrm{x}$ & $\mathrm{z}$ & pitch & roll \\
\hline 0105416 L3 -L4 & -65.2 & 25.7 & 234.6 & -1.5 & -7.9 & 0.7 & 12.8 & -38.4 & 7.2 & 0.0 & 0105416 L3 -L4 & -89.0 & 24.8 & 182.8 & -1.5 & -7.9 & 0.6 & 10.9 & -47.4 & 6.6 & 0.0 \\
\hline UJ00D35 L3-L4 & 32.6 & -35.8 & 53.7 & 1.5 & -7.6 & -0.5 & 18.2 & -68.3 & 6.5 & 0.0 & UJ00D35 L3-L4 & -112.7 & -30.6 & -95.2 & 3.0 & -7.4 & -0.1 & 13.5 & -82.9 & 6.3 & 0.0 \\
\hline ADS0612051 L5-S1 & 8.8 & 2.8 & 147.9 & 0.4 & -8.0 & 0.1 & 6.1 & -49.4 & 9.8 & 0.0 & ADS0612051 L5-S1 & -85.4 & 3.2 & 27.3 & 1.6 & -7.8 & -1.2 & -1.3 & -55.2 & 10.3 & 0.0 \\
\hline ADS0612051 L3-L4 & 33.9 & -79.1 & 180.2 & 0.1 & -7.4 & -2.9 & -38.6 & -68.5 & 9.3 & 0.0 & ADS0612051 L3-L4 & -59.2 & -89.6 & 148.6 & 1.3 & -7.0 & -3.6 & -39.5 & -79.1 & 9.0 & 0.0 \\
\hline DRT060069 L3-L4 & 75.9 & 5.2 & 250.2 & 0.4 & -7.9 & 0.1 & -34.3 & -73.2 & 7.9 & 0.0 & DRT060069 L3-L4 & -50.7 & -3.1 & 222.9 & 0.5 & -7.9 & -0.1 & -35.9 & -82.8 & 8.5 & 0.0 \\
\hline DRT050786 L3-L4 & -33.1 & -28.1 & 94.8 & 1.4 & -7.9 & -0.5 & 11.9 & -39.5 & 9.6 & 0.0 & DRT050786 L3-L4 & -68.4 & 16.8 & -137.4 & 2.4 & -7.7 & -0.4 & 10.6 & -49.4 & 9.7 & 0.0 \\
\hline UF01A030 L3-L4 & 4.7 & -20.2 & 199.7 & 3.1 & -7.3 & -0.2 & -46.1 & -75.2 & 7.0 & 0.0 & UF01A030 L3-L4 & -102.3 & -19.0 & 109.5 & 3.4 & -7.3 & -0.3 & -46.7 & -86.2 & 6.7 & 0.0 \\
\hline Mean & 8.2 & -18.5 & 165.9 & 0.8 & -7.7 & -0.5 & -10.0 & -58.9 & 8.2 & 0.0 & Mean & -81.1 & -13.9 & 65.5 & 1.5 & -7.6 & -0.7 & -12.6 & -69.0 & 8.2 & $\overline{0.0}$ \\
\hline Standard Deviation & 46.4 & 34.2 & 72.1 & 1.4 & 0.3 & 1.2 & 28.2 & 16.0 & 1.4 & 0.0 & Standard Deviation & 22.7 & 38.5 & 138.9 & 1.7 & 0.4 & 1.4 & 26.9 & 17.4 & 1.6 & 0.0 \\
\hline \multicolumn{11}{|c|}{ P1 } & \multicolumn{11}{|c|}{ P2 } \\
\hline Spine & Fx & Fy & Fz & $\mathrm{Mx}$ & My & $\mathrm{Mz}$ & $\bar{x}$ & $\mathrm{z}$ & pitch & roll & Spine & Fx & Fy & $\mathrm{Fz}$ & Mx & My & $\mathrm{Mz}$ & $\mathrm{x}$ & $\bar{z}$ & pitch & roll \\
\hline 0105416 L3 -L4 & -65.8 & 28.9 & 85.9 & 0.2 & -8.1 & 0.3 & 5.7 & -34.0 & 9.3 & 0.0 & 0105416 L3 -L4 & -145.7 & 31.4 & -22.2 & 0.5 & -8.0 & 0.1 & 3.5 & -44.8 & 8.0 & 0.0 \\
\hline UJ00D35 L3-L4 & -17.4 & -26.0 & -250.1 & 3.9 & -7.0 & 0.3 & 8.9 & -64.5 & 6.0 & 0.0 & UJ00D35 L3-L4 & -115.0 & -23.7 & -296.0 & 4.1 & -6.9 & 0.2 & 3.6 & -76.5 & 4.4 & 0.0 \\
\hline ADS0612051 L5-S1 & -28.1 & 9.2 & 40.8 & 2.4 & -7.7 & 0.0 & -0.1 & -43.7 & 12.3 & 0.0 & ADS0612051 L5-S1 & -162.6 & 18.3 & -264.2 & 6.2 & -4.9 & -1.6 & -9.0 & -50.5 & 8.4 & 0.0 \\
\hline ADS0612051 L3-L4 & -15.0 & -106.1 & -326.1 & 5.9 & -5.3 & -1.3 & -48.8 & -66.2 & 8.1 & 0.0 & ADS0612051 L3-L4 & -124.1 & -97.1 & -293.2 & 6.1 & -4.9 & -1.7 & -48.5 & -75.5 & 7.4 & 0.0 \\
\hline DRT060069 L3-L4 & 22.6 & -1.3 & -110.7 & 0.9 & -8.0 & 0.0 & -43.0 & -72.1 & 8.5 & 0.0 & DRT060069 L3-L4 & -127.3 & -8.8 & -189.7 & 1.5 & -7.9 & 0.0 & -44.4 & -81.8 & 7.8 & 0.0 \\
\hline DRT050786 L3-L4 & -50.9 & 26.0 & -349.5 & 2.9 & -7.4 & -1.1 & 3.6 & -37.1 & 7.9 & 0.0 & DRT050786 L3-L4 & -121.0 & 20.6 & -380.6 & 2.8 & -7.4 & -1.7 & 2.6 & -46.7 & 6.1 & 0.0 \\
\hline UF01A030 L3-L4 & -40.2 & -22.3 & -191.4 & 6.7 & -4.4 & -0.4 & -55.8 & -73.4 & 7.0 & 0.0 & UF01A030 L3-L4 & -121.0 & -27.0 & -201.0 & 6.4 & -4.8 & -0.7 & -56.2 & -84.1 & 5.9 & 0.0 \\
\hline Mean & -27.8 & -13.1 & -157.3 & 3.3 & -6.8 & -0.3 & -18.5 & -55.9 & 8.4 & 0.0 & Mean & -130.9 & -12.3 & -235.3 & 3.9 & -6.4 & -0.8 & -21.2 & -65.7 & 6.9 & 0.0 \\
\hline Standard Deviation & 28.7 & 46.2 & 171.2 & 2.4 & 1.4 & 0.6 & 29.1 & 17.0 & 2.0 & 0.0 & Standard Deviation & 17.0 & 43.7 & 113.8 & 2.4 & 1.5 & 0.9 & 27.3 & 17.5 & 1.4 & 0.0 \\
\hline
\end{tabular}


Table B.4: Nucleotomy Spine Condition Extension Data.

\begin{tabular}{|c|c|c|c|c|c|c|c|c|c|c|c|c|c|c|c|c|c|c|c|c|c|}
\hline \multicolumn{11}{|c|}{ Points along Disc Midline } & \multicolumn{11}{|c|}{ Points in Subjacent Body } \\
\hline \multicolumn{11}{|c|}{$\mathrm{A} 1$} & \multicolumn{11}{|c|}{$\mathrm{A} 2$} \\
\hline Spine & Fx & Fy & $\mathrm{Fz}$ & $\mathrm{Mx}$ & My & $\mathrm{Mz}$ & $\bar{x}$ & $\mathrm{z}$ & pitch & roll & Spine & Fx & Fy & $\mathrm{Fz}$ & $\mathrm{Mx}$ & My & $\mathrm{Mz}$ & $\mathrm{x}$ & $\mathrm{z}$ & pitch & roll \\
\hline 0105416 L3 -L4 & -70.6 & 13.6 & -269.5 & 1.0 & 8.0 & 0.6 & 24.4 & -39.1 & -1.5 & 180.0 & 0105416 L3 -L4 & -46.7 & 14.4 & -288.5 & 0.8 & 8.1 & 0.6 & 20.1 & -50.0 & -1.8 & 180.0 \\
\hline UJ00D35 L3-L4 & -92.6 & -15.4 & -247.8 & -1.4 & 7.9 & -0.3 & 29.1 & -63.9 & -1.7 & 180.0 & UJ00D35 L3-L4 & -55.7 & -7.4 & -244.5 & -1.7 & 8.0 & 0.0 & 23.5 & -86.6 & -2.1 & 180.0 \\
\hline ADS0612051 L5-S1 & -154.6 & 17.2 & -213.5 & 2.4 & 7.2 & 2.9 & 13.8 & -54.6 & -2.3 & 180.0 & ADS0612051 L5-S1 & -115.6 & 20.6 & -169.8 & 2.3 & 7.4 & 2.3 & 2.2 & -60.4 & -3.6 & 180.0 \\
\hline ADS0612051 L3-L4 & -69.3 & -74.0 & -252.1 & 2.1 & 7.6 & -2.2 & -27.9 & -71.5 & -1.4 & 180.0 & ADS0612051 L3-L4 & -26.4 & -76.6 & -227.7 & 2.8 & 7.2 & -2.2 & -29.3 & -81.5 & -1.4 & 180.0 \\
\hline DRT060069 L3-L4 & -95.1 & -10.3 & -329.7 & -0.1 & 8.0 & -0.2 & -25.0 & -74.9 & -1.5 & 180.0 & DRT060069 L3-L4 & -51.4 & -8.0 & -323.4 & 0.0 & 8.0 & -0.1 & -26.3 & -84.5 & -1.6 & 180.0 \\
\hline DRT050786 L3-L4 & -58.4 & -6.3 & -210.7 & 0.4 & 8.0 & -0.7 & 19.8 & -42.9 & -1.9 & 180.0 & DRT050786 L3-L4 & -43.9 & -6.8 & -192.8 & 0.4 & 8.0 & -0.6 & 18.8 & -51.9 & -2.0 & 180.0 \\
\hline UF01A030 L3-L4 & -66.2 & -17.4 & -271.4 & 2.8 & 7.5 & -0.3 & -35.5 & -77.5 & -1.9 & 180.0 & UF01A030 L3-L4 & -30.7 & -13.6 & -260.9 & 2.4 & 7.8 & -0.2 & -36.8 & -89.1 & -1.9 & 180.0 \\
\hline Mean & -86.7 & -13.2 & -256.4 & $\overline{1.0}$ & 7.8 & 0.0 & -0.2 & -60.6 & -1.7 & 180.0 & Mean & -52.9 & -11.1 & -244.0 & 1.0 & 7.8 & 0.0 & -4.0 & -72.0 & -2.1 & 180.0 \\
\hline Standard Deviation & 32.9 & 30.1 & 40.4 & 1.5 & 0.3 & 1.5 & 27.9 & 15.4 & 0.3 & 0.0 & Standard Deviation & 29.6 & 31.6 & 53.2 & 1.6 & 0.4 & 1.3 & 26.1 & 17.2 & 0.7 & 0.0 \\
\hline \multicolumn{11}{|c|}{$\mathrm{C} 1$} & \multicolumn{11}{|c|}{$\mathrm{C} 2$} \\
\hline Spine & Fx & Fy & $\mathrm{Fz}$ & $\mathrm{Mx}$ & $\mathrm{My}$ & $\mathrm{Mz}$ & $\mathrm{x}$ & $\mathrm{z}$ & pitch & roll & Spine & Fx & Fy & $\mathrm{Fz}$ & $\mathrm{Mx}$ & My & $\mathrm{Mz}$ & $\mathrm{x}$ & $\mathrm{z}$ & pitch & roll \\
\hline 0105416 L3 -L4 & -99.3 & 14.4 & -298.2 & 0.8 & 8.0 & 0.5 & 13.4 & -38.4 & -3.0 & 180.0 & 0105416 L3 -L4 & -37.5 & 14.6 & -325.1 & 0.6 & 8.0 & 0.6 & 10.8 & -47.5 & -3.0 & 180.0 \\
\hline UJ00D35 L3-L4 & -97.0 & -9.2 & -298.7 & -1.8 & 7.9 & -0.1 & 18.7 & -68.5 & -2.9 & 180.0 & UJ00D35 L3-L4 & -42.1 & -4.1 & -231.3 & -1.6 & 7.9 & 0.0 & 13.4 & -83.1 & -3.0 & 180.0 \\
\hline ADS0612051 L5-S1 & -150.8 & 16.8 & -199.5 & 2.1 & 7.3 & 2.5 & 6.8 & -49.5 & -3.7 & 180.0 & ADS0612051 L5-S1 & -80.9 & 20.2 & -123.8 & 2.3 & 7.7 & 1.3 & -1.3 & -55.4 & -4.5 & 180.0 \\
\hline ADS0612051 L3-L4 & -54.2 & -72.0 & -296.0 & 2.1 & 7.8 & -1.4 & -38.2 & -68.4 & -2.1 & 180.0 & ADS0612051 L3-L4 & -15.5 & -73.4 & -228.1 & 2.4 & 7.6 & -1.3 & -39.5 & -79.1 & -2.0 & 180.0 \\
\hline DRT060069 L3-L4 & -91.0 & -12.9 & -386.8 & -0.2 & 8.1 & -0.2 & -33.9 & -73.3 & -2.6 & 180.0 & DRT060069 L3-L4 & -44.3 & -10.5 & -331.7 & -0.1 & 8.1 & -0.1 & -35.8 & -82.9 & -2.4 & 180.0 \\
\hline DRT050786 L3-L4 & -47.2 & -6.2 & -214.7 & 0.2 & 8.1 & -0.5 & 12.3 & -40.2 & -2.5 & 180.0 & DRT050786 L3-L4 & -36.7 & -6.8 & -198.2 & 0.2 & 8.0 & -0.5 & 10.6 & -49.5 & -2.7 & 180.0 \\
\hline UF01A030 L3-L4 & -79.1 & -12.6 & -280.5 & 1.9 & 7.9 & 0.0 & -45.6 & -75.4 & -3.1 & 180.0 & UF01A030 L3-L4 & -5.9 & -7.2 & -272.0 & 1.6 & 7.9 & 0.0 & -46.7 & -86.3 & -3.1 & 180.0 \\
\hline Mean & -88.4 & -11.7 & -282.1 & 0.7 & 7.9 & 0.1 & -9.5 & -59.1 & -2.8 & 180.0 & Mean & -37.6 & -9.6 & -244.3 & 0.8 & 7.9 & 0.0 & -12.6 & -69.1 & -3.0 & 180.0 \\
\hline Standard Deviation & 34.3 & 29.4 & 62.0 & 1.4 & 0.3 & 1.2 & 28.2 & 15.9 & 0.5 & 0.0 & Standard Deviation & 23.9 & 30.5 & 73.1 & 1.5 & 0.2 & 0.8 & 26.8 & 17.4 & 0.8 & 0.0 \\
\hline \multicolumn{11}{|c|}{ P1 } & \multicolumn{11}{|c|}{ P2 } \\
\hline Spine & $\mathrm{Fx}$ & Fy & Fz & $\mathrm{Mx}$ & $\mathrm{My}$ & $\mathrm{Mz}$ & $\mathrm{x}$ & $\mathrm{z}$ & pitch & roll & Spine & Fx & Fy & $\mathrm{Fz}$ & $\mathrm{Mx}$ & My & $\mathrm{Mz}$ & $\mathrm{x}$ & $\mathrm{z}$ & pitch & roll \\
\hline 0105416 L3 -L4 & -101.5 & 11.9 & -246.0 & 0.6 & 8.1 & 0.3 & 6.0 & -34.1 & -3.9 & 180.0 & 0105416 L3 -L4 & -2.8 & 14.9 & -185.0 & -0.1 & 8.0 & 0.6 & 3.2 & -45.1 & -4.1 & 180.0 \\
\hline UJ00D35 L3-L4 & -95.9 & -6.1 & -252.7 & -1.2 & 8.0 & 0.0 & 9.0 & -64.9 & -4.1 & 180.0 & UJ00D35 L3-L4 & 23.4 & -3.4 & -81.3 & -0.3 & 8.1 & 0.1 & 3.3 & -76.7 & -4.8 & 180.0 \\
\hline ADS0612051 L5-S1 & -154.5 & 14.9 & -185.3 & 1.5 & 7.8 & 1.0 & 0.3 & -43.9 & -4.8 & 180.0 & ADS0612051 L5-S1 & -158.0 & 15.5 & -217.5 & 2.5 & 7.0 & 3.1 & 9.2 & -50.6 & -3.0 & -180.0 \\
\hline ADS0612051 L3-L4 & -38.2 & -72.5 & -260.0 & 1.6 & 7.9 & -0.7 & -48.7 & -66.3 & -3.0 & 180.0 & ADS0612051 L3-L4 & 14.3 & -71.1 & -240.5 & 2.2 & 7.8 & -0.7 & -48.9 & -75.7 & -2.9 & 180.0 \\
\hline DRT060069 L3-L4 & -57.4 & -9.4 & -321.1 & -0.3 & 8.0 & -0.1 & -42.9 & -72.3 & -3.5 & 180.0 & DRT060069 L3-L4 & 21.5 & -2.1 & -266.8 & -0.4 & 8.2 & -0.1 & -44.7 & -82.0 & -3.5 & 180.0 \\
\hline DRT050786 L3-L4 & -47.5 & -6.3 & -210.1 & -0.1 & 8.0 & -0.4 & 3.7 & -37.8 & -3.4 & 180.0 & DRT050786 L3-L4 & -26.2 & -6.8 & -185.3 & -0.1 & 7.9 & -0.4 & 2.3 & -46.9 & -3.5 & 180.0 \\
\hline UF01A030 L3-L4 & -46.1 & -7.7 & -134.4 & 0.1 & 8.1 & 0.1 & -55.6 & -73.7 & -4.2 & 180.0 & UF01A030 L3-L4 & 52.4 & 0.1 & -95.2 & -0.1 & 8.1 & 0.3 & -56.5 & -84.3 & -4.1 & 180.0 \\
\hline Mean & -77.3 & -10.7 & -230.0 & 0.3 & 8.0 & 0.0 & -18.3 & -56.1 & -3.9 & 180.0 & Mean & -10.8 & -7.6 & -181.7 & 0.5 & 7.9 & 0.4 & -18.9 & -65.9 & -3.7 & 128.6 \\
\hline Standard Deviation & 42.2 & 29.0 & 59.9 & 1.0 & 0.1 & 0.5 & 29.1 & 16.9 & 0.6 & 0.0 & Standard Deviation & 69.3 & 29.4 & 70.2 & 1.3 & 0.4 & 1.3 & 29.4 & 17.5 & 0.7 & 136.1 \\
\hline
\end{tabular}


Table B.5: Compliant Implanted Spine Condition Flexion Data.

\begin{tabular}{|c|c|c|c|c|c|c|c|c|c|c|c|c|c|c|c|c|c|c|c|c|c|}
\hline \multicolumn{11}{|c|}{ Points along Disc Midline } & \multicolumn{11}{|c|}{ Points in Subjacent Body } \\
\hline \multicolumn{11}{|c|}{$\mathrm{A} 1$} & \multicolumn{11}{|c|}{$\mathrm{A} 2$} \\
\hline Spine & Fx & Fy & $\mathrm{Fz}$ & $\mathrm{Mx}$ & My & $\mathrm{Mz}$ & $\bar{x}$ & $\mathrm{z}$ & pitch & roll & Spine & Fx & Fy & Fz & $\mathrm{Mx}$ & My & $\mathrm{Mz}$ & $\mathrm{x}$ & $\mathrm{z}$ & pitch & roll \\
\hline 0105416 L3 -L4 & 9.5 & 12.0 & 240.1 & -2.2 & -7.4 & 0.2 & 23.7 & -38.0 & 3.1 & 0.0 & 0105416 L3 -L4 & -17.6 & 18.5 & 212.2 & -2.2 & -7.4 & 0.4 & 19.6 & -48.8 & $\begin{array}{lll}3.4 & & \end{array}$ & 0.0 \\
\hline UJ00D35 L3-L4 & 36.0 & -21.8 & 195.1 & -0.9 & -7.4 & -0.9 & 29.1 & -62.5 & 3.2 & 0.0 & UJ00D35 L3-L4 & -17.1 & -17.2 & 146.0 & -0.3 & -7.7 & -0.4 & 23.7 & -85.1 & 3.2 & 0.0 \\
\hline ADS0612051 L5-S1 & 8.1 & -0.6 & 105.9 & -0.4 & -7.7 & -0.1 & 13.9 & -53.3 & 4.8 & 0.0 & ADS0612051 L5-S1 & -59.2 & 2.3 & 27.9 & 0.3 & -7.9 & -1.6 & 2.8 & -59.2 & 5.9 & 0.0 \\
\hline ADS0612051 L3-L4 & 29.9 & -53.4 & 188.3 & 0.1 & -7.4 & -1.9 & -25.2 & -69.9 & 5.0 & 0.0 & ADS0612051 L3-L4 & -17.9 & -60.4 & 163.0 & 0.9 & -7.2 & -2.5 & -26.1 & -79.8 & 4.9 & 0.0 \\
\hline DRT060069 L3-L4 & -18.0 & 1.3 & 223.0 & 0.5 & -7.6 & 0.0 & -24.2 & -73.6 & 4.7 & 0.0 & DRT060069 L3-L4 & -47.3 & 1.4 & 176.7 & 0.4 & -7.7 & 0.1 & -25.1 & -83.3 & 4.5 & 0.0 \\
\hline DRT050786 L3-L4 & -5.9 & 5.6 & 214.4 & 0.9 & -7.8 & 0.1 & 16.0 & -40.8 & 3.7 & 0.0 & DRT050786 L3-L4 & -44.4 & 5.6 & 176.5 & 0.7 & -7.8 & 0.1 & 15.5 & -49.8 & 3.4 & 0.0 \\
\hline UF01A030 L3-L4 & -10.9 & -40.2 & 248.6 & -0.5 & -7.6 & -0.8 & -34.1 & -76.3 & 2.3 & 0.0 & UF01A030 L3-L4 & -33.1 & -41.0 & 208.6 & 0.3 & -7.7 & -0.8 & -34.9 & -87.8 & 2.1 & 0.0 \\
\hline Mean & 6.9 & -13.9 & 202.2 & $\overline{-0.4}$ & -7.6 & -0.5 & -0.1 & -59.2 & 3.8 & 0.0 & Mean & -33.8 & -13.0 & 158.7 & 0.0 & -7.6 & -0.7 & -3.5 & -70.6 & 3.9 & $\overline{0.0}$ \\
\hline Standard Deviation & 20.3 & 25.1 & 47.8 & 1.0 & 0.2 & 0.8 & 26.6 & 15.5 & 1.0 & 0.0 & Standard Deviation & 17.0 & 28.4 & 62.3 & 1.0 & 0.2 & 1.1 & 24.6 & 17.3 & 1.3 & 0.0 \\
\hline \multicolumn{11}{|c|}{$\mathrm{C} 1$} & \multicolumn{11}{|c|}{$\mathrm{C} 2$} \\
\hline Spine & Fx & Fy & $\mathrm{Fz}$ & $\mathrm{Mx}$ & $\mathrm{My}$ & $\mathrm{Mz}$ & $\mathrm{x}$ & $\mathrm{z}$ & pitch & roll & Spine & $\mathrm{Fx}$ & Fy & Fz & $\mathrm{Mx}$ & $\mathrm{My}$ & $\mathrm{Mz}$ & $\mathrm{x}$ & $\mathrm{z}$ & pitch & roll \\
\hline 0105416 L3 -L4 & 2.1 & 9.3 & 224.2 & $\overline{-2.0}$ & -7.6 & 0.1 & 12.4 & -37.5 & 4.9 & 0.0 & 0105416 L3 -L4 & -47.9 & 18.6 & 175.0 & -1.8 & -7.7 & 0.2 & 10.5 & -46.3 & 4.8 & 0.0 \\
\hline UJ00D35 L3-L4 & 10.4 & -30.7 & 166.3 & -0.8 & -7.7 & -0.5 & 18.6 & -67.2 & 4.5 & 0.0 & UJ00D35 L3-L4 & -64.9 & -26.7 & 63.7 & 0.0 & -7.9 & -0.2 & 13.8 & -81.5 & 4.4 & 0.0 \\
\hline ADS0612051 L5-S1 & -1.5 & 0.8 & 117.1 & -0.1 & -8.0 & -0.6 & 6.6 & -48.2 & 6.6 & 0.0 & ADS0612051 L5-S1 & -97.8 & 2.9 & -33.7 & 0.8 & -7.6 & -2.5 & -1.0 & -54.0 & 7.1 & 0.0 \\
\hline ADS0612051 L3-L4 & 46.6 & -65.5 & 128.6 & 0.2 & -7.6 & -2.0 & -35.7 & -66.8 & 7.5 & 0.0 & ADS0612051 L3-L4 & -57.9 & -74.0 & 90.0 & 1.1 & -7.4 & -2.5 & -36.6 & -77.5 & 7.3 & 0.0 \\
\hline DRT060069 L3-L4 & -4.5 & -1.7 & 208.7 & 0.5 & -7.8 & 0.0 & -33.2 & -72.0 & 6.9 & 0.0 & DRT060069 L3-L4 & -96.9 & -2.6 & 138.5 & 0.4 & -7.9 & 0.0 & -34.8 & -81.7 & 6.7 & 0.0 \\
\hline DRT050786 L3-L4 & -28.5 & 8.2 & 57.2 & 0.6 & -8.0 & -0.1 & 8.4 & -38.1 & 4.9 & 0.0 & DRT050786 L3-L4 & -99.6 & 8.8 & 24.8 & 0.7 & -8.0 & 0.1 & 7.1 & -47.3 & 4.5 & 0.0 \\
\hline UF01A030 L3-L4 & -6.7 & -45.7 & 248.0 & 0.3 & -7.9 & -0.4 & -44.3 & -74.1 & 3.8 & 0.0 & UF01A030 L3-L4 & -83.5 & -45.7 & 198.0 & 0.7 & -7.9 & -0.4 & -45.0 & -85.0 & 3.6 & 0.0 \\
\hline Mean & 2.6 & -17.9 & 164.3 & $\overline{-0.2}$ & -7.8 & -0.5 & -9.6 & -57.7 & 5.6 & 0.0 & Mean & -78.4 & -17.0 & 93.8 & 0.3 & -7.8 & -0.8 & -12.3 & -67.6 & 5.5 & $\overline{0.0}$ \\
\hline Standard Deviation & 22.8 & 29.5 & 67.8 & 0.9 & 0.2 & 0.7 & 26.8 & 16.0 & 1.4 & 0.0 & Standard Deviation & 21.3 & 33.4 & 83.1 & 1.0 & 0.2 & 1.2 & 25.4 & 17.5 & 1.5 & 0.0 \\
\hline \multicolumn{11}{|c|}{ P1 } & \multicolumn{11}{|c|}{$\mathrm{P} 2$} \\
\hline Spine & $\mathrm{Fx}$ & Fy & $\mathrm{Fz}$ & $\mathrm{Mx}$ & $\mathrm{My}$ & $\mathrm{Mz}$ & $\mathrm{x}$ & $\mathrm{z}$ & pitch & roll & Spine & Fx & Fy & $\mathrm{Fz}$ & $\mathrm{Mx}$ & My & $\mathrm{Mz}$ & $\mathrm{x}$ & $\mathrm{z}$ & pitch & roll \\
\hline 0105416 L3 -L4 & -10.2 & 8.8 & -16.8 & -0.1 & -8.0 & 0.1 & 5.3 & -33.0 & 6.4 & 0.0 & 0105416 L3 -L4 & -120.8 & 16.7 & -156.0 & 0.0 & -8.0 & 0.1 & 3.1 & -43.8 & 5.7 & 0.0 \\
\hline UJ00D35 L3-L4 & -38.6 & -43.0 & -58.4 & -0.3 & -8.0 & -0.2 & 9.2 & -63.3 & 5.8 & 0.0 & UJ00D35 L3-L4 & -121.2 & -30.3 & -190.8 & 0.0 & -8.0 & 0.3 & 4.0 & -75.1 & 4.8 & 0.0 \\
\hline ADS0612051 L5-S1 & -43.7 & 2.8 & -0.1 & 0.5 & -7.8 & -1.6 & 0.3 & -42.5 & 8.6 & 0.0 & ADS0612051 L5-S1 & -174.7 & 0.0 & -248.6 & 2.4 & -6.4 & -4.1 & -8.7 & -49.4 & 6.0 & 0.0 \\
\hline ADS0612051 L3-L4 & -30.1 & -74.4 & -405.6 & 1.8 & -7.7 & -0.8 & -45.8 & -64.5 & 6.6 & 0.0 & ADS0612051 L3-L4 & -122.1 & -66.5 & -419.6 & 2.5 & -7.5 & -0.9 & -45.6 & -73.9 & 6.2 & 0.0 \\
\hline DRT060069 L3-L4 & -83.3 & 0.1 & -256.5 & 0.4 & -8.0 & 0.0 & -41.9 & -71.0 & 7.7 & 0.0 & DRT060069 L3-L4 & -167.0 & -0.2 & -308.9 & 0.5 & -8.0 & 0.0 & -43.3 & -80.6 & 6.3 & 0.0 \\
\hline DRT050786 L3-L4 & -88.0 & 23.4 & -327.5 & 0.2 & -8.0 & -0.6 & 0.1 & -35.5 & 4.5 & 0.0 & DRT050786 L3-L4 & -137.3 & 19.4 & -290.6 & 0.2 & -8.0 & -0.6 & -0.8 & -44.5 & 4.1 & 0.0 \\
\hline UF01A030 L3-L4 & -59.4 & -40.4 & -158.8 & 1.0 & -8.0 & 0.0 & -54.0 & -72.3 & 5.3 & 0.0 & UF01A030 L3-L4 & -153.6 & -41.8 & -185.9 & 1.4 & -7.9 & 0.1 & -54.5 & -82.9 & 4.6 & 0.0 \\
\hline Mean & -50.5 & -17.5 & -174.8 & 0.5 & -7.9 & -0.4 & -18.1 & -54.6 & 6.4 & 0.0 & Mean & -142.4 & -14.7 & -257.2 & 1.0 & -7.7 & -0.7 & -20.8 & -64.3 & 5.4 & 0.0 \\
\hline Standard Deviation & 28.2 & 35.3 & 159.5 & 0.7 & 0.1 & 0.6 & 27.7 & 17.0 & 1.4 & 0.0 & Standard Deviation & 22.8 & 32.2 & 91.3 & 1.1 & 0.6 & 1.6 & 25.8 & 17.6 & 0.9 & 0.0 \\
\hline
\end{tabular}


Table B.6: Compliant Implanted Spine Condition Extension Data.

\begin{tabular}{|c|c|c|c|c|c|c|c|c|c|c|c|c|c|c|c|c|c|c|c|c|c|}
\hline \multicolumn{11}{|c|}{ Points along Disc Midline } & \multirow{2}{*}{\multicolumn{11}{|c|}{ Points in Subjacent Body }} \\
\hline \multicolumn{11}{|c|}{$\mathrm{A} 1$} & \multicolumn{4}{|c|}{$\mathrm{A} 2$} & & & & & & & \\
\hline Spine & Fx & Fy & $\mathrm{Fz}$ & $\mathrm{Mx}$ & My & $\mathrm{Mz}$ & $\bar{x}$ & $\mathrm{z}$ & pitch & roll & Spine & Fx & Fy & $\mathrm{Fz}$ & Mx & My & $\mathrm{Mz}$ & $\mathrm{x}$ & $\mathrm{z}$ & pitch & roll \\
\hline 0105416 L3 -L4 & -75.8 & 9.8 & -300.3 & 1.1 & 8.0 & 0.8 & 23.9 & -38.0 & -2.3 & 180.0 & 0105416 L3 -L4 & -36.5 & 9.8 & -258.1 & 0.8 & 8.0 & 0.8 & 19.7 & -48.9 & -2.6 & 180.0 \\
\hline UJ00D35 L3-L4 & -100.3 & -16.8 & -248.1 & -1.7 & 7.9 & -0.4 & 29.5 & -62.5 & -2.3 & 180.0 & UJ00D35 L3-L4 & -50.4 & -9.2 & -197.1 & -1.4 & 8.0 & -0.1 & 23.8 & -85.2 & -2.9 & 180.0 \\
\hline ADS0612051 L5-S1 & -116.2 & -6.8 & -174.2 & -0.1 & 8.1 & -0.2 & 14.2 & -53.5 & -2.3 & 180.0 & ADS0612051 L5-S1 & -80.2 & -1.4 & -125.9 & -0.5 & 8.0 & 0.1 & 2.5 & -59.2 & -4.1 & 180.0 \\
\hline ADS0612051 L3-L4 & -73.3 & -44.8 & -313.6 & 1.5 & 7.9 & -0.7 & -24.9 & -69.9 & -2.4 & 180.0 & ADS0612051 L3-L4 & -16.5 & -42.9 & -258.3 & 1.6 & 8.0 & -0.4 & -26.3 & -79.8 & -2.4 & -180.0 \\
\hline DRT060069 L3-L4 & -105.6 & -9.8 & -289.7 & 0.0 & 8.0 & -0.2 & -23.9 & -73.8 & -2.1 & 180.0 & DRT060069 L3-L4 & -57.9 & -8.1 & -260.0 & 0.0 & 8.1 & -0.1 & -25.2 & -83.4 & -2.2 & 180.0 \\
\hline DRT050786 L3-L4 & -55.3 & 12.5 & -258.9 & -0.5 & 7.5 & -2.8 & 16.3 & -40.7 & -3.7 & 180.0 & DRT050786 L3-L4 & 13.0 & 15.3 & -154.5 & -1.0 & 7.6 & -2.3 & 15.4 & -49.6 & -3.5 & 180.0 \\
\hline UF01A030 L3-L4 & -91.7 & -32.5 & -279.5 & -0.1 & 8.0 & -0.6 & -33.8 & -76.2 & -2.6 & 180.0 & UF01A030 L3-L4 & -19.8 & -29.0 & -244.5 & 0.0 & 8.0 & -0.5 & -35.0 & -87.8 & -2.6 & 180.0 \\
\hline Mean & -88.3 & -12.7 & -266.3 & $\overline{0.0}$ & 7.9 & -0.6 & 0.2 & -59.2 & -2.5 & 180.0 & Mean & -35.5 & -9.4 & -214.0 & -0.1 & 8.0 & -0.4 & -3.6 & -70.6 & -2.9 & 128.6 \\
\hline Standard Deviation & 21.2 & 20.9 & 46.5 & 1.0 & 0.2 & 1.1 & 26.6 & 15.5 & 0.5 & 0.0 & Standard Deviation & 30.8 & 20.6 & 55.6 & 1.0 & 0.2 & 0.9 & 24.7 & 17.3 & 0.7 & 136.1 \\
\hline \multicolumn{11}{|c|}{$\mathrm{C} 1$} & \multicolumn{11}{|c|}{$\mathrm{C} 2$} \\
\hline Spine & Fx & Fy & $\mathrm{Fz}$ & $\mathrm{Mx}$ & $\mathrm{My}$ & $\mathrm{Mz}$ & $\mathrm{x}$ & $\mathrm{z}$ & pitch & roll & Spine & Fx & Fy & $\mathrm{Fz}$ & $\mathrm{Mx}$ & My & $\mathrm{Mz}$ & $\mathrm{x}$ & $\mathrm{z}$ & pitch & roll \\
\hline 0105416 L3 -L4 & -44.5 & 10.8 & -182.8 & 0.4 & 8.1 & 0.6 & 12.9 & -37.5 & -4.0 & 180.0 & 0105416 L3 -L4 & 19.1 & 12.0 & -155.8 & 0.2 & 8.0 & 0.8 & 10.4 & -46.5 & -3.9 & 180.0 \\
\hline UJ00D35 L3-L4 & -95.5 & -16.5 & -210.8 & -1.6 & 7.9 & -0.2 & 19.0 & -67.2 & -3.8 & 180.0 & UJ00D35 L3-L4 & 0.8 & -5.2 & -115.8 & -1.2 & 7.9 & 0.0 & 13.8 & -81.6 & -4.5 & 180.0 \\
\hline ADS0612051 L5-S1 & -148.1 & -6.6 & -199.6 & -0.4 & 8.0 & 0.0 & 7.2 & -48.5 & -3.6 & 180.0 & ADS0612051 L5-S1 & -58.9 & -1.5 & -85.5 & -0.9 & 8.0 & 0.1 & -0.9 & -54.2 & -5.1 & 180.0 \\
\hline ADS0612051 L3-L4 & -19.1 & -44.5 & -231.9 & 0.8 & 8.1 & -0.3 & -35.2 & -66.8 & -3.5 & 180.0 & ADS0612051 L3-L4 & 35.8 & -37.8 & -139.0 & 0.6 & 8.1 & -0.1 & -36.5 & -77.4 & -3.2 & 180.0 \\
\hline DRT060069 L3-L4 & -93.2 & -13.9 & -167.1 & -0.4 & 8.0 & -0.1 & -32.8 & -72.2 & -3.0 & 180.0 & DRT060069 L3-L4 & -25.0 & -3.5 & -203.0 & -0.3 & 8.0 & 0.0 & -34.7 & -81.8 & -3.3 & 180.0 \\
\hline DRT050786 L3-L4 & -10.6 & 3.8 & -79.4 & -1.2 & 7.3 & -3.1 & 8.9 & -38.1 & -4.5 & 180.0 & DRT050786 L3-L4 & 59.6 & 14.4 & -45.8 & -1.3 & 7.6 & -2.5 & 7.2 & -47.3 & -4.4 & 180.0 \\
\hline UF01A030 L3-L4 & -73.5 & -35.6 & -116.3 & -1.3 & 7.9 & -0.3 & -43.8 & -74.2 & -3.6 & 180.0 & UF01A030 L3-L4 & 18.1 & -26.9 & -72.7 & -1.3 & 8.0 & -0.2 & -44.9 & -85.0 & -3.8 & 180.0 \\
\hline Mean & -69.2 & -14.7 & -169.7 & -0.5 & 7.9 & -0.5 & -9.1 & -57.8 & -3.7 & 180.0 & Mean & 7.1 & -6.9 & -116.8 & -0.6 & 7.9 & -0.3 & -12.3 & -67.7 & -4.0 & 180.0 \\
\hline Standard Deviation & 48.4 & 19.9 & 54.2 & 0.9 & 0.3 & 1.2 & 26.8 & 16.0 & 0.4 & 0.0 & Standard Deviation & 39.3 & 19.2 & 53.9 & 0.8 & 0.2 & 1.0 & 25.3 & 17.5 & 0.7 & 0.0 \\
\hline \multicolumn{11}{|c|}{$\mathrm{P} 1$} & \multicolumn{11}{|c|}{$\mathrm{P} 2$} \\
\hline Spine & $\mathrm{Fx}$ & Fy & Fz & $\mathrm{Mx}$ & $\mathrm{My}$ & $\mathrm{Mz}$ & $\mathrm{x}$ & $\mathrm{z}$ & pitch & roll & Spine & Fx & Fy & Fz & $\mathrm{Mx}$ & My & $\mathrm{Mz}$ & $\mathrm{x}$ & $\mathrm{z}$ & pitch & roll \\
\hline 0105416 L3 -L4 & -22.8 & 14.0 & -15.2 & -0.3 & 8.0 & 0.5 & 5.6 & -33.1 & -4.5 & 180.0 & 0105416 L3 -L4 & 54.7 & 15.8 & 21.2 & -0.6 & 8.0 & 0.7 & 2.7 & -44.0 & -4.4 & 180.0 \\
\hline UJ00D35 L3-L4 & -54.2 & -12.8 & -61.7 & -0.9 & 8.0 & 0.0 & 9.4 & -63.5 & -4.9 & 180.0 & UJ00D35 L3-L4 & 66.3 & -10.0 & 101.3 & 0.1 & 8.0 & -0.3 & 3.6 & -75.2 & -5.1 & 180.0 \\
\hline ADS0612051 L5-S1 & -124.3 & -10.1 & -144.2 & -1.1 & 7.9 & 0.1 & 0.7 & -42.8 & -5.0 & 180.0 & ADS0612051 L5-S1 & -29.4 & -6.9 & -31.6 & -1.3 & 7.8 & 0.2 & -8.9 & -49.5 & -6.1 & 180.0 \\
\hline ADS0612051 L3-L4 & 46.5 & -37.6 & -31.4 & -0.6 & 8.1 & 0.0 & -45.7 & -64.6 & -4.5 & 180.0 & ADS0612051 L3-L4 & 90.1 & -34.9 & -27.6 & -0.3 & 8.0 & 0.4 & -45.9 & -74.0 & -4.2 & 180.0 \\
\hline DRT060069 L3-L4 & -35.5 & -11.0 & 21.7 & -0.8 & 8.0 & 0.0 & -41.8 & -71.2 & -3.7 & 180.0 & DRT060069 L3-L4 & 57.5 & -6.6 & 75.6 & -0.7 & 8.1 & -0.1 & -43.6 & -80.9 & -3.7 & 180.0 \\
\hline DRT050786 L3-L4 & 60.7 & 8.0 & 161.3 & -1.6 & 7.4 & -2.5 & 0.3 & -35.6 & -4.6 & 180.0 & DRT050786 L3-L4 & 120.0 & 10.8 & 191.1 & -1.6 & 7.5 & -2.2 & -1.1 & -44.6 & -4.3 & 180.0 \\
\hline UF01A030 L3-L4 & -8.7 & -29.5 & 139.9 & -2.3 & 7.7 & 0.0 & -53.8 & -72.4 & -3.2 & 180.0 & UF01A030 L3-L4 & 76.8 & -22.9 & 185.4 & -2.3 & 7.7 & 0.0 & -54.8 & -83.0 & -3.2 & 180.0 \\
\hline Mean & -19.8 & -11.3 & 10.1 & -1.1 & 7.9 & -0.3 & -17.9 & -54.8 & -4.4 & 180.0 & Mean & 62.3 & -7.8 & 73.6 & -1.0 & 7.9 & -0.2 & -21.1 & -64.5 & -4.4 & 180.0 \\
\hline Standard Deviation & 62.4 & 18.4 & 108.9 & 0.7 & 0.2 & 1.0 & 27.7 & 17.0 & 0.7 & 0.0 & Standard Deviation & 46.2 & 17.7 & 92.3 & 0.8 & 0.2 & 0.9 & 25.8 & 17.6 & 0.9 & 0.0 \\
\hline
\end{tabular}


Table B.7: Non-Compliant Implanted Spine Condition Flexion Data.

\begin{tabular}{|c|c|c|c|c|c|c|c|c|c|c|c|c|c|c|c|c|c|c|c|c|c|}
\hline \multicolumn{11}{|c|}{ Points along Disc Midline } & \multicolumn{11}{|c|}{ Points in Subjacent Body } \\
\hline \multicolumn{11}{|c|}{$\mathrm{A} 1$} & \multicolumn{11}{|c|}{$\mathrm{A} 2$} \\
\hline Spine & $\mathrm{Fx}$ & Fy & Fz & $\mathrm{Mx}$ & My & $\mathrm{Mz}$ & $\mathrm{x}$ & $\mathrm{z}$ & pitch & roll & Spine & $\mathrm{Fx}$ & Fy & $\mathrm{Fz}$ & $\mathrm{Mx}$ & My & $\mathrm{Mz}$ & $\mathrm{x}$ & $\mathrm{z}$ & pitch & roll \\
\hline 0105416 L3 -L4 & 4.9 & 5.3 & 211.3 & -1.9 & -7.6 & 0.2 & 24.8 & -43.0 & $\begin{array}{lll}4.9 & & \end{array}$ & 0.0 & 0105416 L3 -L4 & -39.2 & 13.2 & 206.3 & -2.1 & -7.7 & 0.5 & 20.8 & -53.8 & 5.5 & 0.0 \\
\hline UJ00D35 L3-L4 & 47.3 & -20.6 & 189.3 & -0.7 & -7.6 & -1.4 & 32.8 & -32.2 & 4.3 & 0.0 & UJ00D35 L3-L4 & -18.9 & -16.8 & 158.2 & -0.2 & -7.8 & -0.7 & 27.4 & -54.9 & 4.6 & 0.0 \\
\hline ADS0612051 L5-S1 & 18.5 & 3.0 & 133.3 & -1.1 & -7.8 & -1.3 & 15.3 & -55.7 & 6.6 & 0.0 & ADS0612051 L5-S1 & -80.0 & -19.2 & -11.9 & 0.5 & -7.0 & -4.0 & 4.2 & -61.7 & 8.1 & 0.0 \\
\hline ADS0612051 L3-L4 & 45.1 & -2.9 & 202.0 & -1.0 & -7.8 & -0.3 & 36.7 & -37.3 & 5.6 & 0.0 & ADS0612051 L3-L4 & -15.8 & -10.6 & 188.2 & -0.8 & -7.8 & -0.9 & 35.7 & -47.2 & 5.8 & 0.0 \\
\hline DRT060069 L3-L4 & 36.0 & -0.2 & 227.7 & -0.1 & -7.7 & 0.0 & 30.8 & -39.6 & 4.1 & 0.0 & DRT060069 L3-L4 & -25.3 & -1.3 & 203.7 & -0.1 & -7.8 & 0.0 & 29.9 & -49.2 & 4.3 & 0.0 \\
\hline DRT050786 L3-L4 & -12.9 & -9.2 & 235.7 & 0.0 & -7.9 & 0.0 & 13.5 & -52.3 & 5.4 & 0.0 & DRT050786 L3-L4 & -65.5 & -9.7 & 168.8 & 0.0 & -8.0 & 0.0 & 12.9 & -61.4 & 5.0 & 0.0 \\
\hline UF01A030 L3-L4 & 2.7 & -4.3 & 259.4 & -2.6 & -7.4 & 0.0 & 23.3 & -77.2 & 3.0 & 0.0 & UF01A030 L3-L4 & -27.5 & -5.5 & 224.4 & -2.0 & -7.6 & 0.0 & 22.5 & -88.8 & 3.0 & 0.0 \\
\hline Mean & 20.2 & -4.1 & 208.4 & -1.1 & -7.7 & -0.4 & 25.3 & -43.0 & 4.2 & 0.0 & Mean & -38.9 & -7.1 & 162.5 & -0.7 & -7.7 & -0.7 & 21.9 & -59.6 & 5.2 & $\overline{0.0}$ \\
\hline Standard Deviation & 23.2 & 8.7 & 40.3 & 0.9 & 0.2 & 0.7 & 8.8 & 24.8 & 2.2 & 0.0 & Standard Deviation & 24.6 & 10.9 & 80.2 & 1.0 & 0.3 & 1.5 & 10.7 & 14.0 & 1.6 & 0.0 \\
\hline \multicolumn{11}{|c|}{$\mathrm{Cl}$} & \multicolumn{11}{|c|}{$\mathrm{C} 2$} \\
\hline Spine & $\mathrm{Fx}$ & Fy & $\mathrm{Fz}$ & $\mathrm{Mx}$ & My & $\mathrm{Mz}$ & $\mathrm{x}$ & $\mathrm{z}$ & pitch & roll & Spine & $\mathrm{Fx}$ & Fy & $\mathrm{Fz}$ & $\mathrm{Mx}$ & My & $\mathrm{Mz}$ & $\mathrm{x}$ & $\mathrm{z}$ & pitch & roll \\
\hline 0105416 L3 -L4 & -8.8 & 15.1 & 215.1 & -2.0 & -7.7 & 0.8 & 13.8 & -42.4 & 7.4 & 0.0 & 0105416 L3 -L4 & \begin{tabular}{c|c|}
-93.8 \\
\end{tabular} & 17.9 & 142.0 & -2.0 & -7.7 & 1.0 & 11.7 & -51.3 & 7.6 & 0.0 \\
\hline UJ00D35 L3-L4 & 23.2 & -25.8 & 183.7 & -0.1 & -7.8 & -1.2 & 22.4 & -36.9 & 6.2 & 0.0 & UJ00D35 L3-L4 & -81.7 & -17.3 & 61.5 & 0.4 & -8.1 & -0.1 & 17.5 & -51.3 & 6.3 & 0.0 \\
\hline ADS0612051 L5-S1 & -2.2 & -3.8 & 100.4 & -1.0 & -7.6 & -2.2 & 8.2 & -50.8 & 8.8 & 0.0 & ADS0612051 L5-S1 & -129.0 & -31.8 & -156.9 & 1.9 & -5.5 & -5.5 & 0.8 & -56.7 & 8.2 & 0.0 \\
\hline ADS0612051 L3-L4 & 61.5 & -6.6 & 195.1 & -0.9 & -7.9 & -0.5 & 26.2 & -34.1 & 8.0 & 0.0 & ADS0612051 L3-L4 & -86.1 & -11.7 & 68.7 & -0.6 & -7.9 & -1.2 & 25.3 & -44.8 & 8.3 & 0.0 \\
\hline DRT060069 L3-L4 & 34.6 & -2.1 & 117.1 & -0.8 & -7.9 & 0.0 & 21.8 & -38.0 & 5.8 & 0.0 & DRT060069 L3-L4 & -90.6 & -3.5 & -27.8 & -1.3 & -7.8 & 0.1 & 20.2 & -47.6 & 5.9 & 0.0 \\
\hline DRT050786 L3-L4 & -112.0 & 10.3 & -266.4 & -0.6 & -8.0 & 0.1 & 5.9 & -49.6 & 6.0 & 0.0 & DRT050786 L3-L4 & -164.0 & 11.1 & -332.9 & -0.7 & -8.0 & 0.1 & 4.6 & -58.9 & 5.1 & 0.0 \\
\hline UF01A030 L3-L4 & -25.1 & -1.1 & 81.9 & -3.0 & -7.4 & 0.1 & 13.2 & -75.1 & 4.5 & 0.0 & UF01A030 L3-L4 & -106.3 & -5.4 & 1.6 & -2.4 & -7.7 & 0.1 & 12.5 & -85.9 & 4.5 & 0.0 \\
\hline Mean & -3.3 & -4.9 & 68.6 & -1.1 & -7.8 & $\begin{array}{l}-0.6 \\
\end{array}$ & 16.3 & -47.4 & 6.5 & 0.0 & Mean & -107.3 & -5.8 & -34.8 & \begin{tabular}{c|c|}
-0.7 \\
\end{tabular} & -7.5 & \begin{tabular}{c|}
-0.8 \\
\end{tabular} & 13.2 & -56.7 & 6.6 & 0.0 \\
\hline Standard Deviation & 61.0 & 11.8 & 170.3 & 1.0 & 0.2 & 0.9 & 8.4 & 15.2 & 1.6 & 0.0 & Standard Deviation & 29.6 & 16.8 & 161.4 & 1.4 & 0.9 & 2.2 & 8.6 & 13.8 & 1.5 & 0.0 \\
\hline \multicolumn{11}{|c|}{ P1 } & \multicolumn{11}{|c|}{$\mathrm{P} 2$} \\
\hline Spine & $\mathrm{Fx}$ & Fy & $\mathrm{Fz}$ & $\mathrm{Mx}$ & My & $\mathrm{Mz}$ & $\mathrm{x}$ & $\mathrm{z}$ & pitch & roll & Spine & $\mathrm{Fx}$ & Fy & $\mathrm{Fz}$ & $\mathrm{Mx}$ & My & $\mathrm{Mz}$ & $\mathrm{x}$ & $\mathrm{z}$ & pitch & roll \\
\hline 0105416 L3 -L4 & -62.3 & 19.6 & -246.8 & -0.9 & -7.8 & 1.5 & 6.6 & -37.9 & 8.5 & 0.0 & 0105416 L3 -L4 & $\begin{array}{l}-193.8 \\
\end{array}$ & 17.6 & -476.0 & -0.3 & -7.9 & 1.7 & 4.3 & -49.3 & 7.0 & 0.0 \\
\hline UJ00D35 L3-L4 & -35.8 & -27.8 & -170.7 & 0.6 & -8.0 & -0.1 & 12.9 & -33.0 & 7.5 & 0.0 & UJ00D35 L3-L4 & -170.8 & -16.6 & -370.7 & 0.2 & -8.0 & 0.1 & 7.7 & -44.9 & 5.8 & 0.0 \\
\hline ADS0612051 L5-S1 & -69.7 & -28.6 & -170.7 & 0.8 & -6.5 & -4.7 & 2.0 & -45.2 & 9.6 & 0.0 & ADS0612051 L5-S1 & -154.4 & -30.8 & -256.6 & 3.2 & -4.6 & -5.7 & -7.0 & -52.0 & 4.3 & 0.0 \\
\hline ADS0612051 L3-L4 & -99.1 & 0.0 & -515.0 & 0.8 & -6.5 & 0.0 & 16.0 & -31.9 & 4.8 & 0.0 & ADS0612051 L3-L4 & -169.8 & -0.1 & -509.9 & 0.7 & -6.4 & 0.0 & 16.3 & -41.3 & 4.2 & 0.0 \\
\hline DRT060069 L3-L4 & -99.1 & -1.0 & -418.2 & -2.9 & -7.4 & 0.5 & 13.1 & -36.9 & 4.4 & 0.0 & DRT060069 L3-L4 & -172.8 & -0.3 & -437.1 & -2.7 & -7.5 & 0.5 & 11.7 & -46.5 & 3.3 & 0.0 \\
\hline DRT050786 L3-L4 & -154.3 & 7.8 & -510.3 & -0.7 & -7.9 & -0.6 & -2.4 & -47.1 & 3.4 & 0.0 & DRT050786 L3-L4 & -150.4 & 9.0 & -469.4 & -0.7 & -8.0 & -0.4 & -3.3 & -56.2 & 2.9 & 0.0 \\
\hline UF01A030 L3-L4 & -124.9 & -6.1 & -513.8 & -1.5 & -7.9 & 0.1 & 3.5 & -73.2 & 3.8 & 0.0 & UF01A030 L3-L4 & -140.1 & -6.8 & -458.8 & -1.3 & -8.0 & 0.1 & 3.0 & -83.8 & 3.3 & 0.0 \\
\hline Mean & -92.2 & -5.2 & -363.6 & -0.5 & -7.4 & -0.5 & 7.4 & -43.6 & 6.0 & 0.0 & Mean & -164.6 & -4.0 & -425.5 & -0.1 & -7.2 & -0.5 & 4.7 & -53.5 & 4.4 & 0.0 \\
\hline Standard Deviation & 40.0 & 17.7 & 162.3 & 1.4 & 0.7 & 2.0 & 6.8 & 14.3 & 2.5 & 0.0 & Standard Deviation & 17.7 & 16.1 & 86.0 & 1.9 & 1.3 & 2.4 & 8.1 & 14.2 & 1.5 & 0.0 \\
\hline
\end{tabular}


Table B.8: Non-Compliant Implanted Spine Condition Extension Data.

\begin{tabular}{|c|c|c|c|c|c|c|c|c|c|c|c|c|c|c|c|c|c|c|c|c|c|}
\hline \multicolumn{11}{|c|}{ Points along Disc Midline } & \multicolumn{11}{|c|}{ Points in Subjacent Body } \\
\hline \multicolumn{11}{|c|}{$\mathrm{A} 1$} & \multicolumn{11}{|c|}{$\mathrm{A} 2$} \\
\hline Spine & Fx & Fy & $\mathrm{Fz}$ & $\mathrm{Mx}$ & My & $\mathrm{Mz}$ & $\mathrm{x}$ & $\mathrm{z}$ & pitch & roll & Spine & Fx & Fy & $\mathrm{Fz}$ & $\mathrm{Mx}$ & My & $\mathrm{Mz}$ & $\mathrm{x}$ & $\mathrm{z}$ & pitch & roll \\
\hline 0105416 L3 -L4 & -85.5 & 13.9 & -221.7 & 0.0 & 7.9 & 1.2 & 25.3 & -43.0 & -1.4 & 180.0 & 0105416 L3 -L4 & -55.9 & 14.5 & -266.1 & 0.0 & 8.1 & 1.2 & 20.9 & -53.9 & -1.8 & 180.0 \\
\hline UJ00D35 L3-L4 & -105.8 & -10.8 & -231.5 & -1.9 & 7.9 & -0.2 & 33.3 & -32.2 & -1.6 & 180.0 & UJ00D35 L3-L4 & -94.4 & -9.2 & -264.8 & -2.1 & 7.9 & -0.2 & 27.6 & -55.1 & -2.0 & 180.0 \\
\hline ADS0612051 L5-S1 & -107.9 & 10.8 & -154.2 & 2.1 & 6.8 & -3.7 & 15.9 & -56.1 & -2.7 & 180.0 & ADS0612051 L5-S1 & -17.6 & 13.9 & -52.9 & 0.3 & 7.7 & -2.4 & 4.3 & -61.9 & -3.7 & 180.0 \\
\hline ADS0612051 L3-L4 & -132.1 & 10.6 & -427.4 & 0.5 & 8.0 & 0.7 & 37.2 & -37.3 & -2.1 & 180.0 & ADS0612051 L3-L4 & -106.5 & 11.5 & -396.8 & 0.1 & 8.0 & 0.9 & 35.8 & -47.2 & -2.3 & 180.0 \\
\hline DRT060069 L3-L4 & -110.6 & -1.2 & -331.4 & -3.3 & 7.3 & 0.3 & 31.1 & -39.6 & -1.8 & 180.0 & DRT060069 L3-L4 & -62.4 & -1.0 & -321.6 & -3.5 & 7.3 & 0.3 & 29.8 & -49.2 & -2.0 & 180.0 \\
\hline DRT050786 L3-L4 & -90.4 & -2.8 & -360.2 & -1.1 & 7.8 & -1.7 & 13.8 & -52.4 & -2.8 & 180.0 & DRT050786 L3-L4 & -33.7 & -5.9 & -257.9 & -1.1 & 7.8 & -1.8 & 12.5 & -50.4 & -2.4 & 180.0 \\
\hline UF01A030 L3-L4 & -118.9 & -5.5 & -399.7 & -1.8 & 7.8 & 0.1 & 23.7 & -77.2 & -2.3 & 180.0 & UF01A030 L3-L4 & -33.2 & 7.1 & -328.2 & -2.3 & 7.6 & 0.9 & 19.1 & -54.8 & -2.8 & 180.0 \\
\hline Mean & -107.3 & 2.1 & -303.7 & -0.8 & 7.7 & -0.5 & 25.8 & -48.3 & -2.1 & 180.0 & Mean & -57.7 & 4.4 & -269.8 & -1.3 & 7.8 & -0.2 & 21.4 & -53.2 & -2.4 & 180.0 \\
\hline Standard Deviation & 15.9 & 9.5 & 102.3 & 1.8 & 0.4 & 1.7 & 8.7 & 15.3 & 0.5 & 0.0 & Standard Deviation & 33.0 & 9.7 & 107.6 & 1.5 & 0.3 & 1.4 & 10.8 & 4.9 & 0.7 & 0.0 \\
\hline \multicolumn{11}{|c|}{$\mathrm{Cl}$} & \multicolumn{11}{|c|}{$\mathrm{C} 2$} \\
\hline Spine & $\mathrm{Fx}$ & Fy & $\mathrm{Fz}$ & $\mathrm{Mx}$ & My & $\mathrm{Mz}$ & $\mathrm{x}$ & $\mathrm{z}$ & pitch & roll & Spine & Fx & Fy & $\mathrm{Fz}$ & $\mathrm{Mx}$ & My & $\mathrm{Mz}$ & $\mathrm{x}$ & $\mathrm{z}$ & pitch & roll \\
\hline 0105416 L3 -L4 & -88.9 & 14.3 & -244.4 & 0.1 & 7.9 & 1.0 & 14.1 & -42.5 & -2.3 & 180.0 & 0105416 L3 -L4 & -34.2 & 16.1 & -220.0 & -0.2 & 7.9 & 1.1 & 11.6 & -51.4 & -2.6 & 180.0 \\
\hline UJ00D35 L3-L4 & -129.4 & -7.8 & -276.3 & -2.0 & 7.8 & -0.2 & 22.7 & -37.1 & -2.6 & 180.0 & UJ00D35 L3-L4 & -72.3 & -5.6 & -256.3 & -2.1 & 7.8 & -0.3 & 17.5 & -51.5 & -3.3 & 180.0 \\
\hline ADS0612051 L5-S1 & -67.7 & 14.8 & -105.2 & 1.2 & 7.4 & -3.2 & 8.7 & -51.2 & -3.8 & 180.0 & ADS0612051 L5-S1 & -1.6 & 11.9 & -29.0 & 0.2 & 7.7 & -2.5 & 0.6 & -56.8 & -4.3 & 180.0 \\
\hline ADS0612051 L3-L4 & -116.5 & 17.3 & -380.7 & 0.0 & 8.1 & 0.9 & 26.8 & -34.2 & -3.3 & 180.0 & ADS0612051 L3-L4 & -28.5 & 18.6 & -263.6 & -0.3 & 8.2 & 0.9 & 25.5 & -44.8 & -3.4 & 180.0 \\
\hline DRT060069 L3-L4 & -88.5 & -2.6 & -293.2 & -3.9 & 7.0 & 0.4 & 22.2 & -38.1 & -3.4 & 180.0 & DRT060069 L3-L4 & 7.0 & -2.3 & -190.2 & -3.7 & 7.2 & 0.1 & 20.3 & -47.6 & -3.7 & 180.0 \\
\hline DRT050786 L3-L4 & 2.1 & -5.2 & -79.6 & -1.6 & 7.8 & -1.4 & 6.3 & -49.7 & -3.6 & 180.0 & DRT050786 L3-L4 & 68.1 & -6.3 & -19.8 & -1.5 & 7.8 & -1.2 & 4.7 & -58.9 & -3.5 & 180.0 \\
\hline UF01A030 L3-L4 & -59.0 & -3.3 & -158.1 & -3.0 & 7.4 & 0.1 & 13.6 & -75.1 & -3.9 & 180.0 & UF01A030 L3-L4 & 57.1 & 13.5 & -114.7 & -3.2 & 7.3 & 1.5 & 9.2 & -52.0 & -4.4 & 180.0 \\
\hline Mean & -78.3 & 3.9 & -219.6 & -1.3 & 7.6 & -0.3 & 16.4 & -46.8 & -3.3 & 180.0 & Mean & -0.6 & 6.5 & -156.2 & -1.6 & 7.7 & 0.0 & 12.8 & -51.9 & -3.6 & 180.0 \\
\hline Standard Deviation & 43.3 & 10.9 & 109.3 & 1.8 & 0.4 & 1.5 & 7.7 & 14.0 & 0.6 & 0.0 & Standard Deviation & 50.2 & 10.8 & 102.7 & 1.5 & 0.4 & 1.4 & 8.8 & 4.9 & 0.6 & 0.0 \\
\hline \multicolumn{11}{|c|}{ P1 } & \multicolumn{11}{|c|}{ P2 } \\
\hline Spine & $F x$ & Fy & $\mathrm{Fz}$ & $\mathrm{Mx}$ & My & $\mathrm{Mz}$ & $\mathrm{x}$ & $\mathrm{z}$ & pitch & roll & Spine & $\mathrm{Fx}$ & Fy & $\mathrm{Fz}$ & $\mathrm{Mx}$ & My & $\mathrm{Mz}$ & $\mathrm{x}$ & $\mathrm{z}$ & pitch & roll \\
\hline 0105416 L3 -L4 & -75.7 & 13.9 & -155.3 & -0.2 & 8.0 & 0.8 & 6.8 & -38.2 & -3.1 & 180.0 & 0105416 L3 -L4 & 2.0 & 15.1 & -78.9 & -0.7 & 8.0 & 0.8 & 4.0 & -49.5 & -3.4 & 180.0 \\
\hline UJ00D35 L3-L4 & -119.5 & -2.3 & -196.9 & -1.2 & 7.9 & -0.4 & 13.1 & -33.3 & -3.9 & 180.0 & UJ00D35 L3-L4 & -6.0 & 1.9 & -34.9 & -0.8 & 8.0 & -0.7 & 7.3 & -45.1 & -4.7 & 180.0 \\
\hline ADS0612051 L5-S1 & -37.9 & 14.1 & -51.0 & 0.5 & 7.5 & -3.0 & 2.1 & -45.5 & -4.5 & 180.0 & ADS0612051 L5-S1 & 15.1 & 9.8 & 10.9 & -0.3 & 7.7 & -2.3 & -7.4 & -52.2 & -5.1 & 180.0 \\
\hline ADS0612051 L3-L4 & -22.8 & 22.5 & -124.5 & -0.6 & 8.0 & 0.2 & 16.4 & -32.0 & -4.7 & 180.0 & ADS0612051 L3-L4 & 37.1 & 7.7 & -105.3 & -0.5 & 8.1 & 0.0 & 16.1 & -41.4 & -4.6 & 180.0 \\
\hline DRT060069 L3-L4 & 37.5 & -4.5 & 99.9 & -2.7 & 7.6 & 0.0 & 13.2 & -37.1 & -4.1 & 180.0 & DRT060069 L3-L4 & 105.7 & -4.8 & 161.9 & -2.3 & 7.7 & -0.1 & 11.4 & -46.7 & -3.8 & 180.0 \\
\hline DRT050786 L3-L4 & 77.0 & -7.2 & 166.4 & -2.1 & 7.7 & -1.1 & -2.2 & -47.3 & -3.7 & 180.0 & DRT050786 L3-L4 & 111.8 & -10.6 & 183.8 & -1.9 & 7.8 & -1.1 & -4.0 & -45.3 & -3.3 & 180.0 \\
\hline UF01A030 L3-L4 & 79.1 & 6.3 & 243.2 & -3.4 & 7.2 & 0.8 & 0.3 & -39.4 & -4.3 & 180.0 & UF01A030 L3-L4 & 132.1 & 5.0 & 253.0 & -3.2 & 7.3 & 1.2 & -0.7 & -50.0 & -3.8 & 180.0 \\
\hline Mean & -8.9 & 6.1 & -2.6 & -1.4 & 7.7 & -0.4 & 7.1 & -39.0 & -4.0 & 180.0 & Mean & 56.8 & 3.5 & 55.8 & -1.4 & 7.8 & -0.3 & 3.8 & -47.2 & -4.1 & 180.0 \\
\hline Standard Deviation & 76.4 & 11.2 & 172.1 & 1.4 & 0.3 & 1.3 & 7.3 & 5.7 & 0.6 & 0.0 & Standard Deviation & 57.9 & 8.8 & 141.9 & 1.1 & 0.3 & 1.2 & 8.5 & 3.6 & 0.7 & 0.0 \\
\hline
\end{tabular}




\section{VITA}

Elizabeth Jean Sander was born in Belleville, Illinois on June 22, 1982. She attended Belleville Township High School-West and graduated in May of 2000. In August of the same year, she began her studies in biomedical engineering at Saint Louis University in St. Louis, Missouri. During the summer after her junior year, she had the opportunity to be an intern at Apath, LLC in Creve Coeur, Missouri. While there, she expanded her knowledge in biologics laboratory techniques. Upon graduation with her Bachelor of Science degree in May of 2004, she accepted a full time lab technologist position at the same company. After two years of working in biologics and virology, she decided that she wanted to return to her engineering training. Therefore, she began her graduate studies in August of 2006 at the University of Tennessee Health Science Center under Dr. Denis DiAngelo. While in attendance, she received the 2007 Outstanding Master's Student Award and the 2007 Student Service award from the Department of Biomedical Engineering and Imaging. Her research entitled "Kinematic Demands of Nucleus Arthroplasty Technology" also won the student competitions at the $24^{\text {th }}$ Southern BME Conference and the 2008 Graduate Student Research Day.

Elizabeth currently resides in Memphis, Tennessee, where she works as a Product Development Engineer for Wright Medical Technology. 